\title{
POLYSPHERICAL COORDINATE SYSTEMS ON ORBIT SPACES WITH APPLICATIONS TO BIOMOLECULAR SHAPE
}

\author{
DANIEL B. DIX
}

\begin{abstract}
A general theory of molecular internal coordinates of valence type is presented based on the concept of a Z-system. The Z-system can be considered as a discrete mathematical generalization of the Z-matrix (a molecular geometry file format familiar to chemists) which avoids the principal disadvantage of Z-matrices. Z-matrices are usually only employed for small molecules because there is no easy way to glue two Z-matrices together to get the Zmatrix of a larger molecule. It is shown that Z-matrices are simply Z-systems together with additional extraneous structures and that the Z-systems for any two molecules can be naturally glued together to obtain a Z-system for the combined molecule. A general mathematical framework suitable for the detailed study of molecular geometry is introduced and applied to five and sixmembered molecular rings. A classification of shapes of hexagons with opposite sides and angles congruent is given with explicit parameterizations of the flexible and rigid solutions. The entire mathematical formalism generalizes to a theory of polyspherical coordinate systems on orbit spaces of the group of $n$-dimensional rigid motions acting on finite collections of points in $n$-dimensional Euclidean space. The $n$-dimensional Z-system is a new discrete structure related to abstract simplicial complexes, graded posets, and iterated line graphs. Complete proofs of all the $n$-dimensional results are given, and connections to other areas of mathematics are noted.
\end{abstract}

\section{Contents}

1. Introduction 2

2. Z-systems 10

2.1. Rigid motions, Coordinate Transformations 10

2.2. Orbit Spaces 11

2.3. Simplices 11

2.4. Graph Theory 12

2.5. Z-systems 13

2.6. Iterated Line Graph Construction $\quad 13$

2.7. Fences and Paths in Z-systems $\quad 15$

3. Polyspherical Coordinates 17

3.1. Internal Coordinates Defined 17

3.2. Sites and Conforming Poses 21

3.3. Coordinate Transformations 23

3.4. Labeling the Site Network 26

Date: February 28, 2006.

Key words and phrases. molecular shape, conformational analysis, Z-matrix, Z-system, abstract simplex, spanning tree, line graph, iterated line graph, graded poset, polyspherical coordinates, internal coordinates, valence coordinates, orbit spaces, diagonal action, principal bundle, kinematics, pentagon, hexagon, flexible, rigid. 
3.5. Polyspherical Coordinate Mapping 27

4. The Main Theorems 27

4.1. The Coordinatization Theorem 27

4.2. Scope of Z-system Coordinates 33

5. Z-Matrices $\quad 35$

5.1. Unlabeled Z-matrices Defined 35

5.2. Relating Z-matrices and Z-systems 36

5.3. Labeled Z-matrices $\quad 39$

6. Biomolecular Shape $\quad 40$

6.1. Terminology for Molecular Z-systems 40

6.2. Dihedrals and Impropers 41

6.3. Tethering 43

6.4. Gluing 45

6.5. Five-Membered Rings 48

6.6. Bridging Algorithm, Ring Closure Equations 50

6.7. Hexagons with two-fold symmetry $\quad 53$

$\begin{array}{ll}\text { References } & 58\end{array}$

\section{INTRODUCTION}

Rapid progress in molecular biology has resulted in the exposure of most scientifically literate people to pictures of large biological molecules, such as DNA or proteins. The shapes of these molecules are essential to their functions within living cells [59], [64], [14], [80], [11]. The standard data structure for storage and retrieval of molecular shapes is a "pdb file" [74], which consists primarily of a (very long) list of the Cartesian coordinates of each of the atoms in the molecule. Such data files obviously contain extraneous information, such as that related to the overall position and orientation of the molecule. Moreover, direct visual inspection of the data in this file yields no useful information; one must use computer programs to inspect or interact with the molecule in any meaningful way. This approach to biomolecular structure would lead the mathematician to think of a biomolecule in terms of its configuration, i.e. a mapping $R: \mathcal{N} \rightarrow \mathbb{R}^{3}$ defined on the set $\mathcal{N}$ of atom names which assigns a position $\mathbf{R}_{i}$ to each $i \in \mathcal{N}$. The group $G_{a}=\mathbb{R}^{3} \times \mathrm{SO}(3)$ of rigid motions of $\mathbb{R}^{3}$ acts on such configurations by the geometrically obvious rule: $[(\mathbf{b}, A) R]_{i}=\mathbf{b}+A \mathbf{R}_{i}$ for all $i \in \mathcal{N}$ and all $(\mathbf{b}, A) \in G_{a}$. An orbit $G_{a} R=\left\{(\mathbf{b}, A) R \mid(\mathbf{b}, A) \in G_{a}\right\}$ is here called a shape. Chemists sometimes use the word conformation for a collection of freely interconvertible shapes; in less precise usage "conformation" and "shape" are often synonymous. Focusing on the shape obviously eliminates (in a mathematically trivial way) the extraneous information concerning the overall position and orientation (see [38], [58], [52]), but it does nothing else to add mathematical insight into the nature of biomolecular structure.

Any bijective mapping which assigns a list of coordinates to each shape from a certain set is called an internal coordinate system. If $|\mathcal{N}|=N$ then there should be $3 N-6$ independent internal coordinates assigned to each non-collinear shape. Internal coordinate systems have been studied by chemists and molecular physicists for a long time because of their importance for simplifying the study of molecular 
vibrations, scattering processes, and geometry optimization [6], [7], [16], [20], [28], $[31],[32],[67],[70],[76],[77],[90]$. It is possible to devise internal coordinate systems which use only distances as coordinates; this approach is related to distance geometry [18] and rigidity theory [36], [85]. For attempts in two dimensions to utilize both distances and angles as internal coordinates, see [88], [89]. Internal coordinate systems which are based on collections of bond lengths, bond angles, and torsion angles [70], [82], are loosely called valence coordinates by chemists, but until recently [31] there has been little effort to understand exactly which combinations of these valence coordinates form "good" internal coordinate systems. In fact, the recent appearance of [31] illustrates the fact that the term "valence coordinate system" has never been rigorously defined. This problem is becoming more urgent now that some molecular dynamics simulations are being done in internal coordinates [67], [41], [56]. Mazur and Abagyan have proposed a mathematical structure which they call a "BKS tree", originally proposed by Eyring [28], which reflects the inherent structure of biomolecules more closely than a pdb file does. This structure however involves a linear ordering of $\mathcal{N}$, which from our point of view (see below) is a liability. There is no chemical or biological basis for choosing a particular linear ordering of $\mathcal{N}$.

An important contribution was made in the late 1960s by the quantum chemist John Pople (who later won the Nobel Prize). In the early computational studies of the electronic structure of small molecules, and in particular in the Ph.D dissertation of his student M. A. Gordon [37], a file format was devised for entering the molecular shape into the computer program. Gordon's program employed many matrices, each named by a letter of the alphabet, and the 26th matrix contained the molecular geometry data read from the file. This file format gained the name "Z-matrix" [42], [70]. Z-matrices found increasing use during subsequent developments in computational quantum chemistry, and at least two main variants of this file format have emerged: the Gaussian version [29] and the Mopac version [17]. But these both have the same basic structure, which also involves a linear ordering of $\mathcal{N}$. Because the Z-matrix is not widely known among mathematicians, we will give a (Gaussian style) Z-matrix for the molecule methanol $\mathrm{CH}_{3} \mathrm{OH}$ (see Figures 2 and 3$)$ :

$\begin{array}{cccccccc} & j=0 & j=1 & L & j=2 & \theta & j=3 & \varphi \\ i=1 & C & & & & & & \\ i=2 & O & C & 1.5 & & & & \\ i=3 & H_{1} & C & 1.0 & O & 109.5 & & \\ i=4 & H_{2} & C & 1.0 & O & 109.5 & H_{1} & 120 \\ i=5 & H_{3} & C & 1.0 & O & 109.5 & H_{1} & -120 \\ i=6 & H & O & 1.0 & C & 109.5 & H_{1} & 60\end{array}$

The column and row labels are not part of the Z-matrix, but have been inserted for ease of referencing. Let $A_{i j}$ denote the atom name in row $i$ and column $j$. There are six atoms in methanol with the names $\mathcal{N}=\left\{C, O, H_{1}, H_{2}, H_{3}, H\right\}$, and these are specified in the six rows of the Z-matrix in column $j=0$. Row $i=1$ simply defines the new atom $\{C\}$. Row $i=2$ indicates that the new atom $\{O\}$ should be placed at a distance of $L=1.5$ angstroms from the (previously defined) atom $\{C\}$. This also defines the new atom pair, or bond, $\{O, C\}$. Row $i=3$ indicates that the new atom $\left\{H_{1}\right\}$ should be located at a distance of $L=1.0$ angstroms from the (previously defined) atom $\{C\}$ along a ray (base point at $\{C\}$ ) which makes 


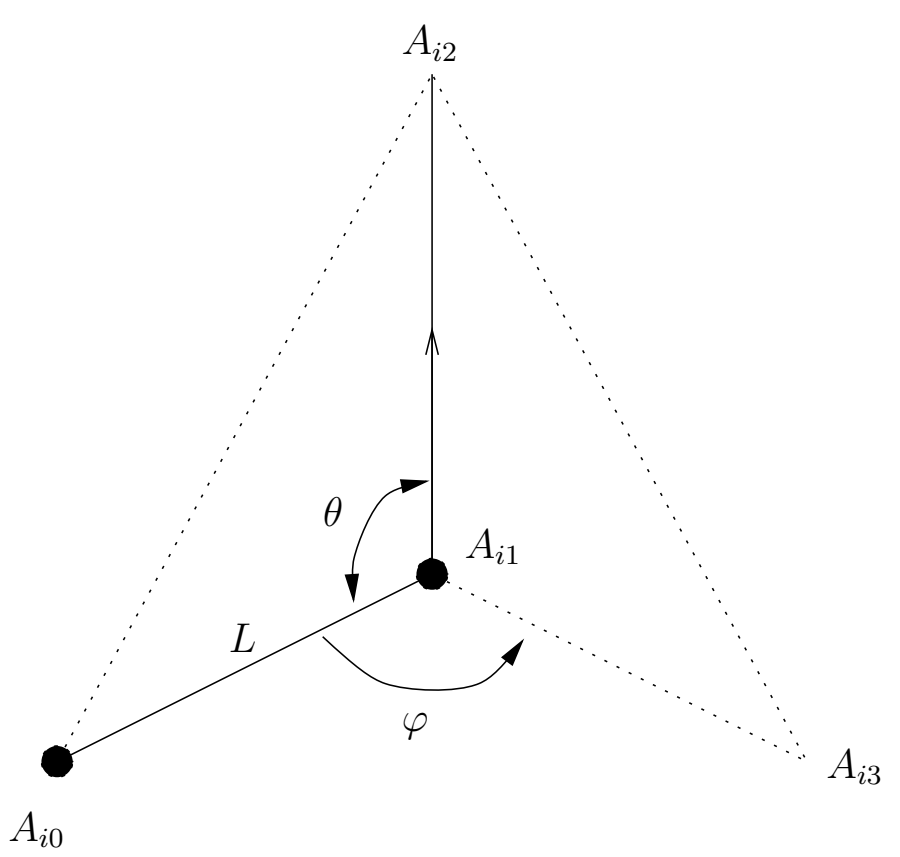

FIGURE 1. Geometric interpretation of Z-matrix coordinates

an angle $\theta=109.5^{\circ}$ with the ray from $\{C\}$ to $\{O\} \quad(\{C, O\}$ being a previously defined bond). This also defines the new bond $\left\{H_{1}, C\right\}$ and the new atom triple, or triangle, $\left\{H_{1}, C, O\right\}$. Rows $i=4,5,6$ all follow the same pattern: the new atom

$\begin{array}{cccccc}\text { new tetrahedron } & \left\{A_{i 0},\right. & A_{i 1}, & A_{i 2}, & \left.A_{i 3}\right\} & \varphi \\ \text { new triangle } & \left\{A_{i 0},\right. & A_{i 1}, & \left.A_{i 2}\right\} & & \theta \\ \text { new bond } & \left\{A_{i 0},\right. & \left.A_{i 1}\right\} & & & \begin{array}{c}\uparrow \\ \text { new atom }\end{array} \\ \left\{A_{i 0}\right\} & & & & \text { coordinates } \\ \text { row } i & A_{i 0} & A_{i 1} & A_{i 2} & A_{i 3} & \\ & & & & & \\ \text { old atom } & & \left\{A_{i 1}\right\} & & & \\ \text { old bond } & & \left\{A_{i 1},\right. & \left.A_{i 2}\right\} & & \\ \text { old triangle } & & \left\{A_{i 1},\right. & A_{i 2}, & \left.A_{i 3}\right\}\end{array}$

$\left\{A_{i 0}\right\}$ is located at a distance $L$ along a ray based at the position of a previously defined atom $\left\{A_{i 1}\right\}$ which forms an angle of $\theta$ (vertex at $\left\{A_{i 1}\right\}$ ) with a ray determined from the previously defined bond $\left\{A_{i 1}, A_{i 2}\right\}$ such that if the ray $\left(A_{i 1}, A_{i 2}\right)$ is an axis of right-handed rotation then the half-plane bounded by this axis and containing the triangle $\left\{A_{i 0}, A_{i 1}, A_{i 2}\right\}$ is rotated through an angle $\varphi$ to coincide with the half-plane bounded by the axis of rotation and containing the previously defined triangle $\left\{A_{i 1}, A_{i 2}, A_{i 3}\right\}$ (see Figure 1 ). As a result of row $i$, the atom $\left\{A_{i 0}\right\}$, the bond $\left\{A_{i 0}, A_{i 1}\right\}$, and the triangle $\left\{A_{i 0}, A_{i 1}, A_{i 2}\right\}$ are newly defined. This complex algorithm takes some practice to get used to, but is easy to use thereafter. $L$ is usually the length of a covalent bond, $\theta$ an angle between two covalent bonds, and 
$\varphi$ is a wedge angle. Thus Z-matrices define particular internal coordinate systems of valence-type.

Z-matrices are now commonly used to specify the geometry of small molecules, but the process becomes too laborious for larger molecules. Typically one would like to form larger molecules by joining smaller ones, as one does with plastic models, but there is no simple algorithm by which a Z-matrix for the larger molecule can be obtained from the Z-matrices for the smaller molecules. Partly this results from the linear ordering of the atoms in the Z-matrix. If one could easily reorder a Z-matrix in a convenient manner then perhaps such a joining algorithm could be devised. We would only wish to consider reordering the atoms in such a way as to not require that the valence coordinates $L, \theta, \varphi$ be recalculated (see section 5.2 for a description of all such reorderings). The computer program Molden [81] advertises a user-defined Z-matrix reordering facility, but it is not clear that it has ever been implemented. Besides, specifying a new ordering is laborious. It would be much better if the underlying mathematical structure, which cannot depend on a linear ordering of $\mathcal{N}$, and which is over-specified by the Z-matrix, could be directly manipulated. This underlying mathematical structure, and its $n$-dimensional generalization, is the subject and principal contribution of this paper. Because of its close connection to the Z-matrix, this structure will be called a Z-system.

The definition of 3-dimensional Z-systems can now be given. An unoriented $Z$-system on $\mathcal{N}$ is a collection $\Gamma$ of subsets of $\mathcal{N}$ satisfying the following conditions.

(1) If $e \in \Gamma$ then $|e| \leq 4$. Define $\Gamma^{k}=\{e \in \Gamma|| e \mid=k+1\}$ for $-1 \leq k \leq 3$.

(2) $\emptyset \in \Gamma^{-1}$ and if $i \in \mathcal{N}$ then $\{i\} \in \Gamma^{0}$.

(3) If $1 \leq k \leq 3$ and $e \in \Gamma^{k}$ then $\left|\left\{v \in \Gamma^{k-1} \mid v \subset e\right\}\right|=2$.

(4) If $1 \leq k \leq 3$ and $v_{1}, v_{2} \in \Gamma^{k-1}$ such that $v_{1} \cup v_{2} \in \Gamma^{k}$ then $v_{1} \cap v_{2} \in \Gamma^{k-2}$.

(5) If $1 \leq k \leq 3$ then the pair $\left(\Gamma^{k-1}, \Gamma^{k}\right)$ is a tree graph, where $\Gamma^{k-1}$ is the set of vertices, $\Gamma^{k}$ is the set of edges, and a vertex $v$ is incident on the edge $e$ if and only if $v \subset e$.

These conditions are not all independent; we state them in this way to emphasize the uniformity of the concept. Condition (3) for $k=1$ and condition (4) with $k=2$ are both consequences of condition (2). For $k=2$ the fact that $\left|\left\{v \in \Gamma^{1} \mid v \subset e\right\}\right| \leq 2$ for all $e \in \Gamma^{2}$ follows from condition (5), $k=1$. In view of condition (5), condition (3) insures that an edge is incident on exactly two vertices, a necessary property of graphs. Conditions (4) and (5) together imply that

- $\left(\Gamma^{1}, \Gamma^{2}\right)$ is a spanning tree in the line graph of $\left(\Gamma^{0}, \Gamma^{1}\right)$, and

- $\left(\Gamma^{2}, \Gamma^{3}\right)$ is a spanning tree in the line graph of $\left(\Gamma^{1}, \Gamma^{2}\right)$.

These properties make it easy to define, visualize, and manipulate unoriented Zsystems graphically, and a computer program exists for this [22].

The unoriented Z-system is easy to extract from a Z-matrix. $\Gamma^{0}$ is obtained from column $j=0$, rows $i \geq 1$ of the Z-matrix. $\Gamma^{1}$ is obtained from columns $j=0$ and $j=1$, rows $i \geq 2$. $\Gamma^{2}$ is obtained from columns $j=0, j=1$, and $j=2$, rows $i \geq 3$. $\Gamma^{3}$ is obtained from columns $j=0, j=1, j=2$, and $j=3$, rows $i \geq 4$. An unoriented Z-system for the molecule methanol corresponding to the Z-matrix listed above is shown in Figure 2. The bond lengths $L$ can be thought of as being associated with elements of $\Gamma^{1}$. Bond angles are associated to elements of $\Gamma^{2}$, and wedge angles are associated to elements of $\Gamma^{3}$ (see the next paragraph). In this way the unoriented Z-system indexes the internal coordinates. 


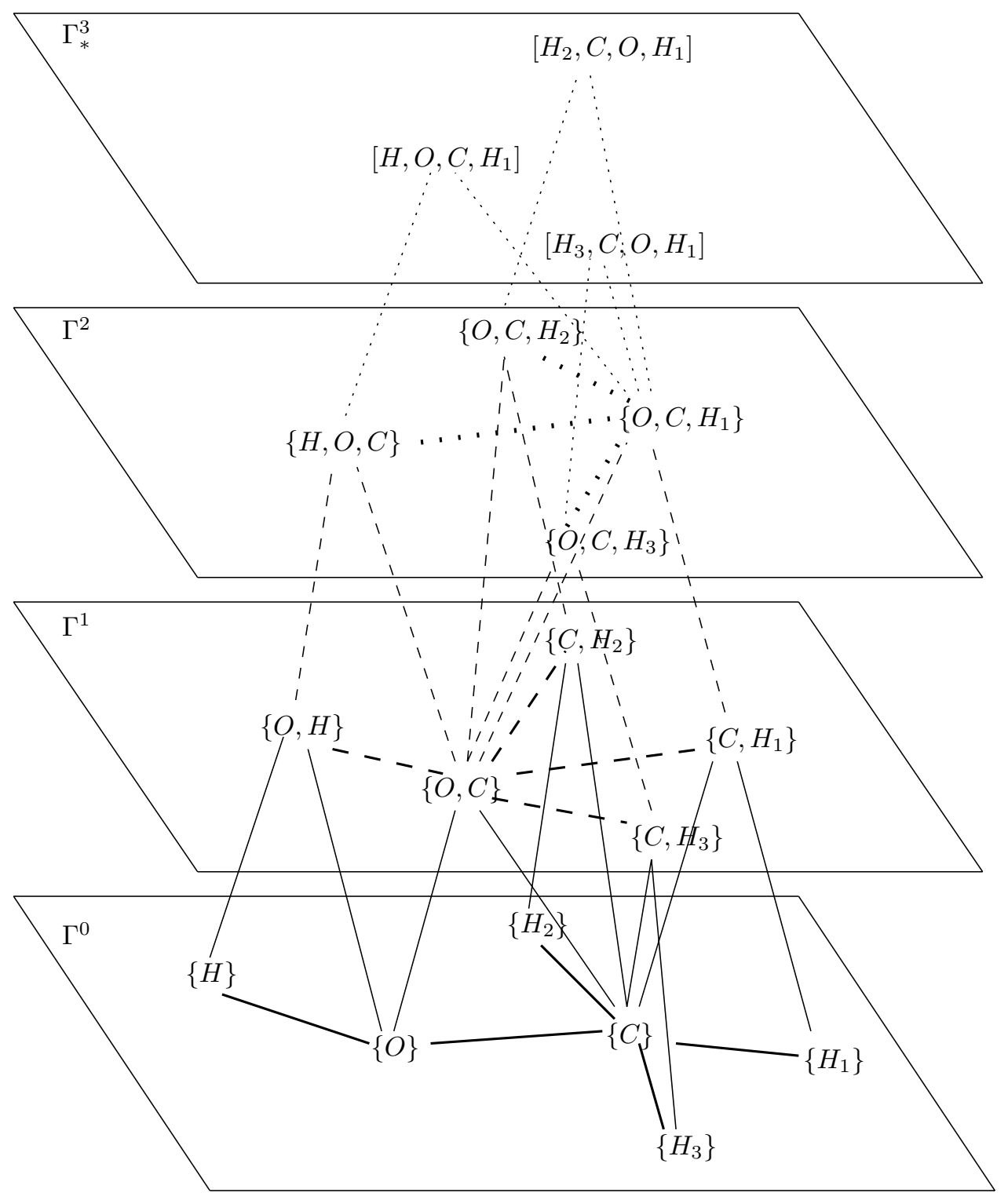

Figure 2. A 3-dimensional Z-system $\Gamma^{*}$ for Methanol. The set $\mathcal{N}=\left\{C, H_{1}, H_{2}, H_{3}, O, H\right\}$ contains the atom names. The tree for $\left(\Gamma^{k-1}, \Gamma^{k}\right), k=1,2,3$, is indicated on the part labeled $\Gamma^{k-1}$, where the edges are indicated by heavier lines of various styles. Above each such line is the element of $\Gamma^{k}$ which is the edge, and it is connected to its two vertices by lighter lines of the same style.

The assertion that wedge angles are associated with elements of $\Gamma^{3}$ needs a slight correction, because an unoriented Z-system does not include certain information which is present in the Z-matrix and is actually necessary to unambiguously describe the molecular shape. For example, row $i=4$ of the Z-matrix for methanol listed 
earlier contains the atom names $\left(H_{2}, C, O, H_{1}\right)$, and the order in which these atoms is listed is important for the precise interpretation of the coordinate $\varphi=120^{\circ}$. The axis of rotation is through the atoms $C$ and $O$, and oriented from $C$ toward $O$. The initial half-plane is determined by this axis and the atom $H_{2}$, whereas the final half-plane is determined by this axis and the atom $H_{1}$. The unoriented Z-system only gives us the four sets:

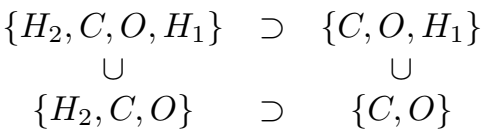

and there are four orderings $\left(i_{0}, i_{1}, i_{2}, i_{3}\right)$ of $\left\{H_{2}, C, O, H_{1}\right\}$ which yield the same subsets by the rule $\left\{i_{0}, i_{1}, i_{2}\right\},\left\{i_{1}, i_{2}, i_{3}\right\}$ and $\left\{i_{1}, i_{2}\right\}$, namely

$$
\begin{array}{ll}
\left(\mathrm{H}_{2}, \mathrm{C}, \mathrm{O}, \mathrm{H}_{1}\right) & \left(\mathrm{H}_{2}, \mathrm{O}, \mathrm{C}, \mathrm{H}_{1}\right) \\
\left(\mathrm{H}_{1}, \mathrm{O}, \mathrm{C}, \mathrm{H}_{2}\right) & \left(\mathrm{H}_{1}, \mathrm{C}, \mathrm{O}, \mathrm{H}_{2}\right) .
\end{array}
$$

Let us assume that the connectivity of the atoms, the bond lengths and the bond angles are fixed as in the above Z-matrix. This data alone does not fix the shape of the molecule. The two 4-tuples in the first column actually determine from the angle $\varphi=120^{\circ}$ the same shape $\mathcal{O}$ of the four atoms. This is because in $\left(H_{1}, O, C, H_{2}\right)$ the orientation of the axis of rotation is reversed and the initial and final half-planes are also reversed in comparison to $\left(H_{2}, C, O, H_{1}\right)$ (c.f. Figure 1). The two 4-tuples in the second column determine from the same angle $\varphi=120^{\circ}$ a shape $\mathcal{O}^{\prime}$ of the four atoms which is the mirror image of the shape $\mathcal{O}$. Chemists say $\mathcal{O}$ and $\mathcal{O}^{\prime}$ have opposite chirality, although that word is most often used for the arrangement of four substituents to a central atom. The concept of chirality has elicited many mathematical treatments [24], [23], [91], [18]. If $\mathbf{R}_{H_{2}}, \mathbf{R}_{C}, \mathbf{R}_{O}, \mathbf{R}_{H_{1}} \in \mathbb{R}^{3}$ denote the position vectors of these four atoms then the chirality of the shape depends on the sign of the following $4 \times 4$ determinant

$$
\begin{aligned}
& \operatorname{det}\left(\begin{array}{cccc}
1 & 1 & 1 & 1 \\
\mathbf{R}_{H_{2}} & \mathbf{R}_{C} & \mathbf{R}_{O} & \mathbf{R}_{H_{1}}
\end{array}\right)=\operatorname{det}\left(\begin{array}{cccc}
1 & 1 & 1 & 1 \\
\mathbf{R}_{C} & \mathbf{R}_{H_{2}} & \mathbf{R}_{H_{1}} & \mathbf{R}_{O}
\end{array}\right) \\
& =\operatorname{det}\left(\begin{array}{cccc}
1 & 0 & 0 & 0 \\
\mathbf{R}_{C} & \mathbf{R}_{H_{2}}-\mathbf{R}_{C} & \mathbf{R}_{H_{1}}-\mathbf{R}_{C} & \mathbf{R}_{O}-\mathbf{R}_{C}
\end{array}\right) \\
& =\left(\mathbf{R}_{H_{2}}-\mathbf{R}_{C}\right) \times\left(\mathbf{R}_{H_{1}}-\mathbf{R}_{C}\right) \cdot\left(\mathbf{R}_{O}-\mathbf{R}_{C}\right),
\end{aligned}
$$

which obviously depends on the order in which the atoms are listed.

So in addition to an unoriented Z-system we need some extra information so that a specification of $\varphi \in(-\pi, \pi]$ will fix a definite chirality of the four atoms involved. Of the two 4-tuples in the first column we find that $\left(H_{1}, O, C, H_{2}\right)$ is an even permutation of $\left(H_{2}, C, O, H_{1}\right)$, whereas both of the 4-tuples in the second column are odd permutations of $\left(H_{2}, C, O, H_{1}\right)$ and even permutations of each other. Thus the extra information we need beyond that contained in the unoriented Z-system is a choice, for each $e \in \Gamma^{3}$, of an equivalence class $e^{*}$ of orderings of $e$, where two orderings are deemed equivalent if one is an even permutation of the other. There are two such equivalence classes for each $e$; if $e^{*}$ is one of them then let $-e^{*}$ denote the other one. Each row of a Z-matrix determines a particular ordering, and hence an equivalence class of orderings. Each ordering of $e$ within $e^{*}$ will lead to the same sign of the determinant exemplified above. If the configuration $R$ is given it is natural to choose $e^{*}$ so that the corresponding determinant is nonnegative and $\varphi \in[0, \pi]$. Mostly however we do not wish to let the choice of $e^{*}$ be determined by 
the configuration, since it can easily change abruptly as the molecule moves; thus we note that $\left(e^{*}, \varphi\right)$ and $\left(-e^{*},-\varphi\right)$ correspond to exactly the same configuration. With the choice of $e^{*}$ fixed in time the quantity $e^{i \varphi}$ varies smoothly on the circle as the molecule moves. Once these choices have been made and included in the set $\Gamma_{*}^{3}$ then we say $\Gamma^{*}=\left(\Gamma \backslash \Gamma^{3}\right) \cup \Gamma_{*}^{3}$ determines an oriented $Z$-system, a terminology which we usually shorten to $Z$-system. Thus wedge angles should be assigned to members of the set $\Gamma_{*}^{3}$.

This particular mathematical formalism for indexing valence coordinates has all the desirable properties that one could reasonably hope to obtain, and the proofs will be given in this paper. We prove that a Z-system $\Gamma^{*}$ determines a well-defined system of internal coordinates and we characterize the set $\mathcal{D}_{C}(\Gamma)$ (the coordinate domain) of shapes non-singularly described as well as the set $\mathcal{D}_{P}\left(\Gamma^{*}\right)$ (the parameter domain) of parameter specifications which correspond to actual shapes in $\mathcal{D}_{C}(\Gamma)$. This is the first general result of this kind to be proved. We also show that Zsystems enjoy very natural joining operations, which we call tethering and gluing. We also precisely capture the relationship between Z-systems and Z-matrices by showing what extra structure we must add to an unoriented Z-system to determine a Z-matrix. We also show how a Z-system comes equipped with a family of sites which together with a specific configuration of the molecule are associated with special Cartesian coordinate systems, or poses, which are well-conformed to the shape of the molecule at each site. Coordinate transformation matrices relating these poses can be related to tree structures inherent in the Z-system, and to the internal coordinates of the molecule. This allows one to systematically study detailed questions about molecular shapes, such as deriving systems of algebraic equations for molecular rings or helical structures, etc. Earlier work related to this aspect is: [33], [19], [26], [40], [61], [78], [79]. We show that any non-collinear configuration of three or more atoms can be non-singularly described by some Zsystem internal coordinate system.

Thus Z-systems provide the first mathematically rigorous and chemically adequate general theory of valence internal coordinates, and they are consistent with the guidelines stated in [31]. Despite assertions in that paper, there do exist valence internal coordinate systems (combinations of bond lengths, bond angles, and wedge angles) which are not Z-system coordinate systems and which fail to satisfy the guideline "bond angle coordinates must all subtend two of the bond length coordinates present in a valid set". For example if the lengths (positive) of bonds $\{1,2\}$ and $\{2,3\}$ and the measure (not 0 or $\pi$ radians) of the angle $\{\{1,2\},\{2,3\}\}$ is given then the length of the "bond" $\{1,3\}$ is thereby determined and is positive, and hence it can be used as part of another angle, such as $\{\{1,3\},\{3,4\}\}$. Thus the question of exactly which valence coordinate systems give rise to a one-to-one correspondence between coordinate and parameter domains as defined in this paper remains open. However since Z-systems are adequate for any non-collinear configuration the practical benefit of considering systems more general than Z-systems and yet with the same coordinate and parameter domains is not likely to be real.

Not every molecular internal coordinate is of valence type. For example the improper Wilson angle (see page 247 of [82]), used in some molecular force fields, is not a Z-system coordinate. This coordinate does not facilitate the description of a wider variety of molecular geometries than addressed by Z-systems. We do not claim that the kinetic energy or the potential energy of a molecule assumes a 
simplified form in Z-system coordinates. In particular there is no easy way to tell from the Z-system coordinates of a molecule whether two atoms, which are more than 4 bonds away from one another in the tree $\left(\Gamma^{0}, \Gamma^{1}\right)$, are too close to one another in space. It also may not be possible to choose a Z-system for a molecule which completely preserves the symmetry of the molecule in a particular configuration.

In combinatorial topology a subset $e \subset \mathcal{N}$ with $|e|=k+1$ is called an abstract $k$-simplex, and an equivalence class of orderings of $e$ with respect to the even permutation equivalence relation discussed above is called an oriented abstract $k$-simplex [71]. However an unoriented Z-system $\Gamma$ is not an abstract simplicial complex since $\Gamma$ will never contain every face of every abstract simplex in $\Gamma$. However $\Gamma$ will be a special type of skeleton complex [4]. Also an unoriented Z-system is a very pleasant new type of graded poset, [84]. However the Z-system concept is not mentioned in any of these guises in [35]. Despite its novelty there is the question of the significance of the Z-system formalism. There are other mathematical formalisms which allow one to achieve some of the above theorems in regard to 3-dimensional molecular shapes (for example one based on iterated line graphs), and the Z-system idea loses its attractiveness if it is just one formalism among many of nearly equal power. From a mathematical point of view the correct formalism should be able to be generalized to the $n$-dimensional case so as to define polyspherical coordinates for orbits of mappings $R: \mathcal{N} \rightarrow \mathbb{R}^{n}$ under the action of $G_{a}=\mathbb{R}^{n} \times \mathrm{SO}(n)$. This is not true for the iterated line graph formalism (see section 2.6). Z-systems and Z-matrices generalize immediately to the $n$-dimensional setting. We will prove that essentially all of the 3-dimensional properties we have discussed above generalize nicely to the $n$-dimensional case. However, gluing of two $n$-dimensional Z-systems is a little more involved than in the 3-dimensional case and we will leave that for later work. The characterization of which $n$-dimensional shapes can be non-singularly described by some $n$-dimensional Z-system is proved by a method quite unlike that which might be at first devised for the 3-dimensional result. Generally we found that the $n$-dimensional proofs, while slightly less concrete, yielded greater insight than the overly simplified proofs which are available only in low dimensional cases. We know of no formalism other than that of Z-systems which has such nice general properties.

Thus the Z-system presents itself as a new construction with interesting possibilities for connections to various fields of pure mathematics, such as combinatorics and graph theory, rigidity theory, combinatorial topology, graded poset theory, real algebraic geometry, geometrical mechanics, kinematics, and the theory of manifolds and principal bundles. Z-system theory is a new development in chemical graph theory [8], [86], [69], [9], [27]. Our definitions of wedge angles agree completely with standard chemical definitions [82], [54], [72], allowing us to describe molecular shapes in widely understood terms. We think that standardized Z-systems can profitably be chosen for each of the monomers of biopolymers, according to the guidelines in [43], [44], [45] and [62], and the Z-systems for the biopolymers will then be determined by our standard gluing operations (see section 6.4). A modification of the Z-matrix incorporating a hierarchical system of atom names might then become an alternate (and immediately informative) storage and retrieval data structure to pdb files for biological macromolecules (gluing would still require software support). Z-systems are especially suitable for biomolecules because these molecules operate at relatively low energies where covalent bonds are difficult to 
break and bond length and bond angle vibrations are of small amplitude. Thus the important modes of flexibility are directly accessible and the invariant geometrical aspects are easily held constant. In this way Z-systems can model important mathematical aspects of biomolecular structure. For a detailed application of Z-systems to a large biomolecular system see [49].

The plan of the rest of this paper is as follows. Section 2 establishes general notation and in particular explains the definition and construction of $n$-dimensional Z-systems. The next section shows how to define internal coordinates given an $n$ dimensional Z-system. Also the network of sites, conforming poses, and coordinate transformation matrices are discussed and the relation to internal coordinates is explained. Then the next section proves the two main theorems. The first main theorem asserts that Z-system style coordinates determine a one-to-one correspondence between shapes in the coordinate domain and parameter specifications in the parameter domain. The second main theorem shows exactly which shapes are in the union of the coordinate domains of all the Z-systems on $\mathcal{N}$, establishing the general scope of utility of these systems of internal coordinates. In the next section we give the definition of $n$-dimensional Z-matrices, and show that there is a one-to-one correspondence between the collection of Z-matrices on $\mathcal{N}$ and the set of triples $(\Gamma, r, \lambda)$, where $\Gamma$ is an unoriented Z-system on $\mathcal{N}, r$ is a site from $\Gamma$, and $\lambda$ is a leaf-picking order in the highest level tree in $\Gamma$. Finally in the last section we further discuss the applications of Z-systems to molecules. We establish terminology specific to that dimension, define the operations of tethering and gluing of Z-systems, give a result on five-membered rings which suggests ways that our results might be generalized, and present a "bridging" algorithm able to concisely explain the set of shapes of a six-membered ring.

\section{Z-SYSTEMS}

2.1. Rigid motions, Coordinate Transformations. We begin with some general notation used throughout the paper. Suppose $\mathbb{R}^{n}$ is equipped with the standard Euclidean inner product, and with the usual orientation. (An orientation of an inner product space is an equivalence class of ordered orthonormal bases, where two ordered orthonormal bases are equivalent if the matrix relating them has unit determinant.) The standard basis $I=\left(\hat{\mathbf{e}}_{1}, \ldots, \hat{\mathbf{e}}_{n}\right)$ is positively oriented and orthonormal. $\mathrm{SO}(n)$ denotes the group of all $n \times n$ real matrices $A$ such that $A^{T} A=A A^{T}=I$ and $\operatorname{det}(A)=1$. $\left(A^{T}\right.$ denotes the transpose of the matrix $A$. $)$ Define $G_{a}=\mathbb{R}^{n} \times \operatorname{SO}(n)$ to be the group with the binary operation $\left(\mathbf{b}_{1}, A_{1}\right)\left(\mathbf{b}_{2}, A_{2}\right)=\left(\mathbf{b}_{1}+A_{1} \mathbf{b}_{2}, A_{1} A_{2}\right)$, identity $(\boldsymbol{\theta}, I)$, and inverse operation $(\mathbf{b}, A)^{-1}=\left(-A^{T} \mathbf{b}, A^{T}\right)$. The group $G_{a}$ has a left action on $\mathbb{R}^{n}$ by the rule $(\mathbf{b}, A) \cdot \mathbf{x}=\mathbf{b}+A \mathbf{x}$. $G_{a}$ is called the group of $n$-dimensional rigid motions; the subscript $a$ stands for active. $G_{a}$ can be identified with the group of all orientation preserving and distance preserving mappings from $\mathbb{R}^{n}$ to itself [10]. Define an $n \times(n+1)$ matrix $\left(\mathbf{e}_{0}, \mathbf{e}_{1}, \ldots, \mathbf{e}_{n}\right)$ to be a pose [61] if $\mathbf{e}_{0} \in \mathbb{R}^{n}$ and $\left(\mathbf{e}_{1}, \ldots, \mathbf{e}_{n}\right)$ is a positively oriented orthonormal basis of $\mathbb{R}^{n}$. Every pose defines a Cartesian coordinate system in $\mathbb{R}^{n}$ with origin $\mathbf{e}_{0}$ in the usual way. Let $\mathcal{P}$ denote the set of all poses. $G_{a}$ acts on $\mathcal{P}$ on the left by the rule $(\mathbf{b}, A)\left(\mathbf{e}_{0}, \mathbf{e}_{1}, \ldots, \mathbf{e}_{n}\right)=\left(\mathbf{b}+A \mathbf{e}_{0}, A \mathbf{e}_{1}, \ldots, A \mathbf{e}_{n}\right)$. This action is transitive and fixed point free. Since $A=\left(A \hat{\mathbf{e}}_{1}, \ldots, A \hat{\mathbf{e}}_{n}\right) \in \mathrm{SO}(n)$ we see that there is a natural identification of $G_{a}$ with $\mathcal{P}$; we will mostly ignore it however. Define $G_{p}$ to be the group 
of all $(n+1) \times(n+1)$ matrices of the form $\left(\begin{array}{cc}1 & \boldsymbol{\theta}^{T} \\ \mathbf{b} & A\end{array}\right)$, where $\boldsymbol{\theta} \in \mathbb{R}^{n}$ is the zero (column) vector, and where $(\mathbf{b}, A) \in G_{a} . G_{p}$ is a group under the operation of ordinary matrix multiplication. In fact the groups $G_{a}$ and $G_{p}$ are isomorphic in the obvious manner. $G_{p}$ acts on $\mathcal{P}$ on the right by ordinary matrix multiplication. This group action is also transitive and fixed point free. We call $G_{p}$ the passive group of coordinate transformations. The left action of $G_{a}$ on $\mathcal{P}$ commutes with the right action of $G_{p}$.

2.2. Orbit Spaces. If $A$ and $B$ are sets then let $A^{B}$ denote the set of all mappings from $B$ to $A$. Let $\mathcal{N}$ be a set of $N$ elements. Elements of the set $\left(\mathbb{R}^{n}\right)^{\mathcal{N}}$ will be called configurations; if $R \in\left(\mathbb{R}^{n}\right)^{\mathcal{N}}$ and $i \in \mathcal{N}$ then instead of $R(i)$ we will write $\mathbf{R}_{i}$. $G_{a}$ acts on $\left(\mathbb{R}^{n}\right)^{\mathcal{N}}$ via the diagonal action, i.e. if $g \in G_{a}, R \in\left(\mathbb{R}^{n}\right)^{\mathcal{N}}$, and $i \in \mathcal{N}$ then $(g \cdot R)_{i}=g \cdot \mathbf{R}_{i}$. Orbits $G_{a} R=\left\{g \cdot R \mid g \in G_{a}\right\}$ of this left action will be called shapes. If a group $G$ acts on the left (resp. right) on a set $X$ then $G \backslash X$ (resp. $X / / G$ ) will denote the set of all orbits $G x$ (resp. $x G$ ) of elements $x \in X$. Define

$$
\mathcal{B}:=\left\{R \in\left(\mathbb{R}^{n}\right)^{\mathcal{N}} \mid \text { if }(\mathbf{b}, A) \in G_{a} \text { and }(\mathbf{b}, A) \cdot R=R \text { then }(\mathbf{b}, A)=(\boldsymbol{\theta}, I)\right\} .
$$

$\mathcal{B}$ is a dense open subset of $\left(\mathbb{R}^{n}\right)^{\mathcal{N}}$ which is invariant under the action of $G_{a}$. This action of the Lie group $G_{a}$ on the manifold $\mathcal{B}$ is fixed point free and proper, and hence general results [2] imply that the orbit space $G_{a} \backslash \mathcal{B}$ is a smooth manifold; $G_{a} \backslash \mathcal{B}$ is sometimes called the shape space [63]. A coordinate chart $\phi: \mathcal{D}_{C}(\phi) \rightarrow$ $\mathcal{D}_{P}(\phi)$ for this manifold is a smooth bijection whose inverse $\phi^{-1}: \mathcal{D}_{P}(\phi) \rightarrow \mathcal{D}_{C}(\phi)$ is also smooth. The open set $\mathcal{D}_{C}(\phi) \subset G_{a} \backslash \mathcal{B}$ is called the coordinate domain of $\phi$. The open set $\mathcal{D}_{P}(\phi) \subset \mathbb{R}^{n N-n(n+1) / 2}$ is called the parameter domain of $\phi$. This paper will define a particular atlas of coordinate charts in the manifold $G_{a} \backslash \mathcal{B}$. Let $\rho: \mathcal{B} \rightarrow G_{a} \backslash \mathcal{B}: R \mapsto G_{a} R$ be the smooth projection mapping. $\rho$ is an example of a principal fiber bundle with (a left acting) structure group $G_{a}$ [51].

2.3. Simplices. If $S$ is any set define $\left(\begin{array}{l}S \\ k\end{array}\right)$ to be the set of all subsets of $S$ with exactly $k$ elements. We call an element of $\left(\begin{array}{c}\mathcal{N} \\ k+1\end{array}\right)$ an abstract $k$-simplex. An element of $\left(\begin{array}{c}\mathbb{R}^{n} \\ k+1\end{array}\right)$ is called a $k$-simplex. If there is no possible confusion we will sometimes call an abstract simplex a simplex. A $k$-simplex $\left\{\mathbf{R}_{0}, \mathbf{R}_{1}, \ldots, \mathbf{R}_{k}\right\}$ is called geometrically independent if the set $\left\{\left(\begin{array}{c}1 \\ \mathbf{R}_{0}\end{array}\right),\left(\begin{array}{c}1 \\ \mathbf{R}_{1}\end{array}\right), \ldots,\left(\begin{array}{c}1 \\ \mathbf{R}_{k}\end{array}\right)\right\}$ is linearly independent in $\mathbb{R}^{n+1}$. If $R \in\left(\mathbb{R}^{n}\right)^{\mathcal{N}}$ and $s=\left\{i_{0}, i_{1}, \ldots, i_{k}\right\}$ is an abstract $k$-simplex then define $R_{s}:=$ $\left\{\mathbf{R}_{i_{0}}, \mathbf{R}_{i_{1}}, \ldots, \mathbf{R}_{i_{k}}\right\}$ to be the associated simplex. For $s \in\left(\begin{array}{c}\mathcal{N} \\ n\end{array}\right)$ define

$$
\mathcal{B}_{s}:=\left\{R \in\left(\mathbb{R}^{n}\right)^{\mathcal{N}} \mid R_{s} \text { is a geometrically independent } n-1 \text { simplex }\right\} .
$$

Lemma. If $N \geq n \geq 2$ then $\mathcal{B}=\cup\left\{\mathcal{B}_{s} \mid s \in\left(\begin{array}{c}\mathcal{N} \\ n\end{array}\right)\right\}$.

Proof. Define $\mathcal{B}^{\prime}:=\cup\left\{\mathcal{B}_{s} \mid s \in\left(\begin{array}{c}\mathcal{N} \\ n\end{array}\right)\right\}$. First, suppose $R \in \mathcal{B}^{\prime}$, so that there is at least one $s \in\left(\begin{array}{c}\mathcal{N} \\ n\end{array}\right)$ such that $R_{s}$ is a geometrically independent $n-1$ simplex. Let $(\mathbf{b}, A)$ be such that $(\mathbf{b}, A) \cdot R=R$. Then $\mathbf{b}+A \mathbf{R}_{i}=\mathbf{R}_{i}$ for all $i \in s$. Thus for $i, j \in s, i \neq j$, we have $A\left(\mathbf{R}_{i}-\mathbf{R}_{j}\right)=\mathbf{R}_{i}-\mathbf{R}_{j}$. Thus the eigenspace of $A$ for the eigenvalue 1 is of dimension at least $n-1$. If $\left(\mathbf{e}_{1}, \ldots, \mathbf{e}_{n}\right)$ is a positively oriented orthonormal basis of $\mathbb{R}^{n}$ such that $A \mathbf{e}_{i}=\mathbf{e}_{i}$ for $i=1, \ldots, n-1$ then we must have $A \mathbf{e}_{n}=\mathbf{e}_{n}$. Thus $A=I$ and consequently $\mathbf{b}=\boldsymbol{\theta}$. Hence $R \in \mathcal{B}$. Therefore $\mathcal{B}^{\prime} \subset \mathcal{B}$. 
For the converse suppose $R \in \mathcal{B}$. Let $i_{0} \in \mathcal{N}$ and $V=\operatorname{span}\left\{\mathbf{R}_{i}-\mathbf{R}_{i_{0}} \mid i \in\right.$ $\left.\mathcal{N} \backslash\left\{i_{0}\right\}\right\}$. If it were the case that the dimension of $V$ is less than or equal to $n-2$ then let $\left(\mathbf{e}_{1}, \ldots, \mathbf{e}_{n}\right)$ be a positively oriented orthonormal basis of $\mathbb{R}^{n}$ such that $V \subset$ $\operatorname{span}\left\{\mathbf{e}_{1}, \ldots, \mathbf{e}_{n-2}\right\}$. By defining a nontrivial rotation in the plane $\operatorname{span}\left\{\mathbf{e}_{n-1}, \mathbf{e}_{n}\right\}$, and extending it to be the identity on $\operatorname{span}\left\{\mathbf{e}_{1}, \ldots, \mathbf{e}_{n-2}\right\}$, we obtain a nontrivial $A \in \mathrm{SO}(n)$ which is the identity on $V$. Then $(\mathbf{b}, A)=\left(\mathbf{R}_{i_{0}}-A \mathbf{R}_{i_{0}}, A\right)$ is a nonidentity element of $G_{a}$ such that $(\mathbf{b}, A) \cdot R=R$, contradicting the fact that $R \in \mathcal{B}$. Thus when $R \in \mathcal{B}$ we have that the dimension of $V$ is greater than or equal to $n-1$. Hence there is a set $s^{\prime}=\left\{i_{1}, \ldots, i_{n-1}\right\} \subset \mathcal{N} \backslash\left\{i_{0}\right\}$ such that $\left\{\mathbf{R}_{i}-\mathbf{R}_{i_{0}} \mid i \in s^{\prime}\right\}$ is linearly independent in $\mathbb{R}^{n}$. A simple argument now shows that if $s=\left\{i_{0}\right\} \cup s^{\prime}$ then $R_{s}$ is a geometrically independent $n-1$ simplex. Thus $R \in \mathcal{B}^{\prime}$, and hence $\mathcal{B} \subset \mathcal{B}^{\prime}$.

Let $\{0: n\}=\{0,1, \ldots, n\}$ and $\operatorname{inj}(A, B)$ denote the set of all injective mappings between sets $A$ and $B$. Let even $(A)$ denote the set of all bijections of the finite linearly ordered set $A$ with itself which determine even permutations. The set $\operatorname{inj}(\{0: n\}, \mathcal{N})$ has a right action of the group even $(\{0: n\})$ by composition of mappings: if $\alpha \in \operatorname{inj}(\{0: n\}, \mathcal{N})$ and $p \in \operatorname{even}(\{0: n\})$ then $\alpha \circ p$ is the result of $p$ acting on $\alpha$. An oriented abstract $n$-simplex $s^{*}$ is member of the orbit space $\operatorname{inj}(\{0: n\}, \mathcal{N}) / / \operatorname{even}(\{0: n\})$, i.e. an equivalence class $s^{*}=[\alpha]=\alpha \cdot \operatorname{even}(\{0: n\})$ of injections $\alpha:\{0: n\} \rightarrow \mathcal{N}$, where two such injections $\alpha$ and $\alpha^{\prime}$ are equivalent if $\alpha^{\prime}=\alpha \circ p$ for some even permutation $p:\{0: n\} \rightarrow\{0: n\}$. If $s^{*}=[\alpha]$ is an oriented abstract $n$-simplex, then we also write $s^{*}=[\alpha(0), \alpha(1), \ldots, \alpha(n)]$ with the understanding that $[\alpha(0), \alpha(1), \ldots, \alpha(n)]=[\alpha(p(0)), \alpha(p(1)), \ldots, \alpha(p(n))]$ for all even permutations $p$. Assigning every injective mapping its range defines a mapping $\operatorname{inj}(\{0: n\}, \mathcal{N}) \rightarrow\left(\begin{array}{c}\mathcal{N} \\ n+1\end{array}\right)$. Since this mapping is invariant under the action of the group even $(\{0: n\})$, it factors through the projection onto the orbit space, giving a mapping $\Upsilon: \operatorname{inj}(\{0: n\}, \mathcal{N}) / / \operatorname{even}(\{0: n\}) \rightarrow\left(\begin{array}{c}\mathcal{N} \\ n+1\end{array}\right)$. Thus $\Upsilon$ maps any oriented abstract $n$-simplex to its underlying abstract $n$-simplex, i.e. $\Upsilon\left(s^{*}\right)=\Upsilon([\alpha(0), \alpha(1), \ldots, \alpha(n)])=\{\alpha(0), \alpha(1), \ldots, \alpha(n)\}=s$.

2.4. Graph Theory. Suppose $V \subset\left(\begin{array}{c}\mathcal{N} \\ k\end{array}\right)$ and $E \subset\left(\begin{array}{c}\mathcal{N} \\ k+1\end{array}\right)$, where $k \geq 1$. We would like to think of the pair $\tau=(V, E)$ as a graph, where $V=\operatorname{vert} \tau$ is the set of vertices, $E=$ edge $\tau$ is the set of edges, and a vertex $v \in V$ is incident on the edge $e \in E$ if and only if $v \subset e$. This is contrary to the traditional definition of graph, which requires that each edge be an element of $\left(\begin{array}{c}V \\ 2\end{array}\right)$, and each edge is incident on its two members. However the essential thing about the traditional definition is that each edge must be incident on exactly two vertices, and an edge should be uniquely determined by its two vertices. We can insure this is true in the case of $(V, E)$ if we impose the additional condition:

- for each $e \in E$ the set $\{v \in V \mid v \subset e\}$ has exactly two elements.

If $e \in E$ and $\{v \in V \mid v \subset e\}=\left\{v_{1}, v_{2}\right\}$ then since $v_{1} \neq v_{2}$ it is automatic that $e=v_{1} \cup v_{2}$, and hence that $e$ is uniquely determined by $v_{1}$ and $v_{2}$. We will use this non-traditional graph idea frequently, but we will always first check the above additional condition. If a pair of sets $(V, E)$ is a graph by the traditional definition, then we will always call it a traditional graph.

For the convenience of the reader we record here several standard [87] graph theory definitions. Consider an ordered list $t=\left(v_{0}, e_{1}, v_{1}, \ldots, v_{m-1}, e_{m}, v_{m}\right)$ where $v_{0}, \ldots, v_{m}$ are vertices of $V$ and $e_{1}, \ldots, e_{m}$ are edges from $E$, such that $e_{i}$ is incident 
on both $v_{i-1}$ and $v_{i}$ for all $i=1,2, \ldots, m . t$ is called a path of length $m$ in $(V, E)$ connecting $v_{0}$ to $v_{m}$ if $v_{0}, v_{1}, \ldots, v_{m}$ are distinct. $t$ is called a cycle if $v_{1}, \ldots, v_{m}$ are distinct, $v_{0}=v_{m}$, and $m \geq 3$. The graph $(V, E)$ is said to be connected if for any two distinct vertices $v, v^{\prime}$ of $V$ there exists a path connecting $v$ to $v^{\prime}$. If a graph contains no cycle it is said to be acyclic. A connected acyclic graph is said to be a tree. A vertex $v$ is said to be a leaf if there is only one edge $e$ incident on $v$. A triple $(V, E, r)$ is called a rooted graph if a distinguished vertex $r$ of $V$ has been chosen; $r$ is called the root vertex, or simply the root. Suppose $\left(V_{1}, E_{1}\right)$ and $\left(V_{2}, E_{2}\right)$ are graphs. We say $\left(V_{1}, E_{1}\right)$ is a subgraph of $\left(V_{2}, E_{2}\right)$ if $V_{1} \subset V_{2}$ and $E_{1} \subset E_{2}$. The subgraph $\left(V_{1}, E_{1}\right)$ is said to be spanning if $V_{1}=V_{2}$. The subgraph $\left(V_{1}, E_{1}\right)$ is said to be induced by $V_{1}$ if $E_{1}$ consists of exactly those elements of $E_{2}$ whose two vertices are actually elements of $V_{1}$. If $(V, E, r)$ is a rooted tree then a leaf-picking order for this rooted tree is an ordering of the elements of the set $V=\left\{v_{1}, v_{2}, \ldots, v_{m}\right\}$ such that $r=v_{1}$ and for every $2 \leq k \leq m$ the vertex $v_{k}$ is a leaf of the subgraph of $(V, E)$ induced by the set $\left\{v_{1}, \ldots, v_{k}\right\}$. Such leaf-picking orders are known to exist for any rooted tree [87].

2.5. Z-systems. Now we are ready to give a general definition of Z-systems.

Definition. An $n$-dimensional unoriented $Z$-system on the set $\mathcal{N}$ is a collection $\Gamma$ of subsets of $\mathcal{N}$ satisfying the following conditions.

(1) If $e \in \Gamma$ then $|e| \leq n+1$. Define $\Gamma^{k}=\{e \in \Gamma|| e \mid=k+1\},-1 \leq k \leq n$.

(2) $\emptyset \in \Gamma^{-1}$ and if $i \in \mathcal{N}$ then $\{i\} \in \Gamma^{0}$, i.e. $\Gamma^{0}=\left(\begin{array}{c}\mathcal{N} \\ 1\end{array}\right)$.

(3) If $1 \leq k \leq n$ and $e \in \Gamma^{k}$ then $\left|\left\{v \in \Gamma^{k-1} \mid v \subset e\right\}\right|=2$.

(4) If $1 \leq k \leq n$ and $v_{1}, v_{2} \in \Gamma^{k-1}$ such that $v_{1} \cup v_{2} \in \Gamma^{k}$ then $v_{1} \cap v_{2} \in \Gamma^{k-2}$.

(5) If $1 \leq k \leq n$ then the pair $\left(\Gamma^{k-1}, \Gamma^{k}\right)$ is a tree graph.

Definition. Suppose $\Gamma$ is an $n$-dimensional unoriented Z-system on the set $\mathcal{N}$ and $\Gamma_{*}^{n}$ is a collection of oriented abstract $n$-simplices such that $\Upsilon$ determines a bijective mapping of $\Gamma_{*}^{n}$ onto $\Gamma^{n}$. Then we say $\Gamma^{*}=\left(\Gamma \backslash \Gamma^{n}\right) \cup \Gamma_{*}^{n}$ is an $n$-dimensional $Z$ system on $\mathcal{N}$ whose underlying unoriented $Z$-system is $\Gamma$.

2.6. Iterated Line Graph Construction. It is not entirely obvious that unoriented Z-systems exist in higher dimensions. But if an unoriented Z-system exists it is obvious that it can be made into a Z-system in many different ways. Thus we will now give an inductive construction proving the existence of unoriented Z-systems in every dimension and showing the degree of flexibility in the concept. To begin, note that the complete graph $\left(\left(\begin{array}{c}\mathcal{N} \\ 1\end{array}\right),\left(\begin{array}{c}\mathcal{N} \\ 2\end{array}\right)\right)$ is connected. In any connected graph it is possible to choose a spanning tree. So choose a spanning tree $\left(\Gamma^{0}, \Gamma^{1}\right)$ as a subgraph of $\left(\Gamma^{0},\left(\begin{array}{c}\mathcal{N} \\ 2\end{array}\right)\right)$. Notice that if $e \in \Gamma^{1}$ is incident on the two vertices $v_{1}, v_{2} \in \Gamma^{0}$ then $v_{1} \cap v_{2}=\emptyset \in \Gamma^{-1}$. Thus a 1-dimensional unoriented Z-system necessarily exists.

Now suppose for $n \geq 2$ that an $(n-1)$-dimensional unoriented Z-system $\Gamma$ has been chosen. Thus, in particular, $\left(\Gamma^{n-2}, \Gamma^{n-1}\right)$ is a tree. Recall that the traditional line graph of $\left(\Gamma^{n-2}, \Gamma^{n-1}\right)$ is defined to be the traditional graph $\left(\Gamma^{n-1}, L_{t}\right)$, where

$$
\begin{aligned}
L_{t} & =\left\{\left\{e_{1}, e_{2}\right\} \mid e_{1}, e_{2} \in \Gamma^{n-1}, e_{1} \neq e_{2} \text {, and there exists } v \in \Gamma^{n-2}\right. \text { such that } \\
& \left.v \subset e_{1} \text { and } v \subset e_{2}\right\} \\
= & \left\{\left\{e_{1}, e_{2}\right\} \mid e_{1}, e_{2} \in \Gamma^{n-1} \text { and } e_{1} \cap e_{2} \in \Gamma^{n-2}\right\} .
\end{aligned}
$$


There is the possibility of giving a non-traditional version $\left(\Gamma^{n-1}, L\right)$ of this traditional graph, where

$$
L=\left\{e_{1} \cup e_{2} \mid e_{1}, e_{2} \in \Gamma^{n-1}, e_{1} \cap e_{2} \in \Gamma^{n-2}\right\},
$$

but we must check the additional condition to insure that $\left(\Gamma^{n-1}, L\right)$ is a graph. This will follow from the following lemma, which proves an even stronger result needed later.

Lemma. Suppose $\left(\Gamma^{k-1}, \Gamma^{k}\right)$ is a tree for $k=1, \ldots, n-1$. Suppose $\tau$ is a tree subgraph of the traditional line graph $\left(\Gamma^{n-1}, L_{t}\right)$ with $m \geq 1$ edges, and $S=\cup\{e \mid$ $e \in \operatorname{vert} \tau\}$. Then for all $0 \leq k \leq n-1$ we have that $\left|\left\{e \in \Gamma^{k} \mid e \subset S\right\}\right|=n+m-k$.

Proof. Define for each $k=0,1, \ldots, n-1$ the sets $\Gamma_{S}^{k}=\left\{s \in \Gamma^{k} \mid s \subset S\right\}$. We want to show that $\left|\Gamma_{S}^{k}\right|=n+m-k$. Clearly $\tau$ is a tree with $m$ edges, and therefore $m+1$ vertices, each of which is a member of $\Gamma^{n-1}$. Hence $\left|\Gamma_{S}^{n-1}\right| \geq m+1$. Now for all $k=1, \ldots, n-1$ we claim that $\left(\Gamma_{S}^{k-1}, \Gamma_{S}^{k}\right)$ is a graph. We must verify the additional condition. To see this suppose $e \in \Gamma_{S}^{k} \subset \Gamma^{k}$. Since $\left(\Gamma^{k-1}, \Gamma^{k}\right)$ is a graph there are exactly two vertices $v_{1}, v_{2} \in \Gamma^{k-1}$ on which $e$ is incident. Since both are subsets of $e \subset S$, both $v_{1}$ and $v_{2}$ are in $\Gamma_{S}^{k-1}$. Clearly there cannot be in $\Gamma_{S}^{k-1}$ any other vertex incident on $e$. Thus $e$ is incident on exactly two vertices in $\Gamma_{S}^{k-1}$. Therefore our claim is demonstrated. Hence $\left(\Gamma_{S}^{n-2}, \Gamma_{S}^{n-1}\right)$ is a subgraph of the tree $\left(\Gamma^{n-2}, \Gamma^{n-1}\right)$, and hence is acyclic. The connected components of this subgraph are all trees, and hence have one more vertex than the number of edges. Consequently $\left|\Gamma_{S}^{k-1}\right| \geq\left|\Gamma_{S}^{k}\right|+1$. By an induction argument we see that $\left|\Gamma_{S}^{k}\right| \geq n+m-k$ for all $k=0, \ldots, n-1$ (we call this inequality the lower bound). Therefore $n+m \leq\left|\Gamma_{S}^{0}\right|$. Let $\left\{e_{0}, e_{1}, \ldots, e_{m}\right\}$ be a leaf-picking order for $\tau$, where the root vertex $e_{0}$ is chosen arbitrarily. Since $e_{0} \in \Gamma^{n-1}$ we have $\left|e_{0}\right|=n$. Now $\left\{e_{0}, e_{1}\right\}$ is an edge in $L_{t}$, and hence $e_{0} \cap e_{1} \in \Gamma^{n-2}$. Thus $\left|e_{0} \cup e_{1}\right|=n+1$. Likewise $e_{2}$ is connected to either $e_{0}$ or $e_{1}$ by an edge in $L_{t}$, and hence $\left|e_{0} \cup e_{1} \cup e_{2}\right| \leq n+2$. Continuing in this fashion we find that $\left|e_{0} \cup e_{1} \cup \cdots \cup e_{m}\right| \leq n+m$. Since $\Gamma^{0}=\left(\begin{array}{c}\mathcal{N} \\ 1\end{array}\right)$ we have $\left|\Gamma_{S}^{0}\right| \leq n+m$ (upper bound). Since the lower bound and the upper bound agree we have $\left|\Gamma_{S}^{0}\right|=n+m$. Since we have already established that $\left|\Gamma_{S}^{k}\right| \leq\left|\Gamma_{S}^{k-1}\right|-1$ (upper bound), an induction argument shows that $\left|\Gamma_{S}^{k}\right|=n+m-k$ for all $k=0,1, \ldots, n-1$, since the upper and lower bounds agree at each value of $k$.

We apply this lemma with the tree $\tau$ consisting of the two vertices $e_{1}$ and $e_{2}$ and a single edge $\left\{e_{1}, e_{2}\right\} \in L_{t},(m=1)$ and $S=e_{1} \cup e_{2}$. We find that $\mid\left\{e \in \Gamma^{n-1} \mid e \subset\right.$ $S\} \mid=n+1-(n-1)=2$. Thus the additional condition is verified, and $\left(\Gamma^{n-1}, L\right)$ is therefore the line graph of the tree $\left(\Gamma^{n-2}, \Gamma^{n-1}\right)$. Since line graphs of connected graphs are connected we can choose a spanning tree $\left(\Gamma^{n-1}, T^{n}\right)$ in $\left(\Gamma^{n-1}, L\right)$. If $\Gamma^{\prime}=\Gamma \cup T^{n}$ then $\Gamma^{\prime}$ is an $n$-dimensional unoriented Z-system.

It is also true that every unoriented Z-system can be obtained by means of the inductive procedure just described. This is because condition (4) of the definition implies that $\left(\Gamma^{k-1}, \Gamma^{k}\right)$ is a spanning tree in the line graph of $\left(\Gamma^{k-2}, \Gamma^{k-1}\right), 2 \leq$ $k \leq n$.

The $n$-dimensional unoriented Z-system $\Gamma$ can be put in one-to-one correspondence with the $n$-tuple $\left(\tau_{1}, \ldots, \tau_{n}\right)$, where $\tau_{1}$ is a spanning tree in the traditional complete graph on $\Gamma^{0}$ and $\tau_{k+1}$ is a spanning tree in the traditional line graph of $\tau_{k}$ for all $k=1, \ldots, n-1$. The simplicity of this alternate approach to unoriented Z-systems makes it a natural object of study by pure mathematicians independent 


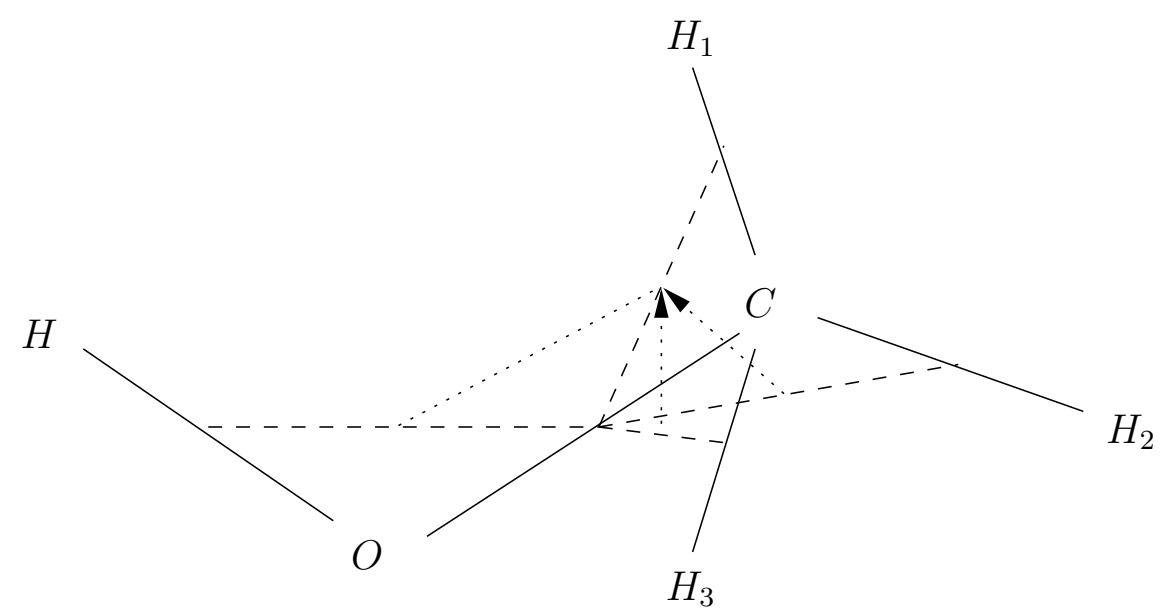

Figure 3. The iterated line graph form (used by IMIMOL [22]) of the same Z-system $\Gamma^{*}$ for Methanol as in Figure 2. Bonds (1simplices, or edges of $\left.\tau_{1}\right)$ are indicated by solid lines. Triangles (2simplices) are in one-to-one correspondence with angles (edges of $\tau_{2}$ ), and are indicated by dashed lines from one vertex bond to the other. Tetrahedra (3-simplices) are in one-to-one correspondence with unordered pairs of angles (edges of $\tau_{3}$ ), and are indicated by curved or straight dotted lines from one vertex angle to the other. Impropers must be given a direction; dihedrals assume their canonical orientation (see section 6 ).

of its applications. Others have also noticed the utility of iterated line graphs for the study of molecular shape [27]. A specification of $\Gamma$ is to look at an unoriented Z-system in the poset picture. A specification of $\left(\tau_{1}, \ldots, \tau_{n}\right)$ is to look at an unoriented Z-system in the iterated line graph picture. These pictures can be used to visualize a Z-system. For example in Figure 2 we give a 3-dimensional Z-system for the molecule methanol in the poset picture. Figure 3 is the same Z-system, but given in the iterated line graph picture. (See section 6 for a discussion of dihedrals and impropers in the 3-dimensional case.) Although the diagrams are easier to draw in the (3-dimensional) iterated line graph picture, the set theoretical nature of the vertices and edges involved becomes quite complex for the higher level trees. Furthermore, rather than orienting the edges of $\tau_{n}$, we must orient the corresponding $n$-simplices in $\Gamma^{n}$. So we commonly use the iterated line graph picture to display 3-dimensional Z-systems, but use the poset picture for statements of theorems and their proofs.

2.7. Fences and Paths in Z-systems. A "top down" construction of a Z-system satisfying certain geometrical constraints will be given in the proof of the theorem of section 4.2. This construction will use the following concept.

Definition. Let $\tau_{1}, \ldots, \tau_{m}$ be $(k+1)$-simplices and $s_{0}, s_{1}, \ldots, s_{m}$ be distinct $k$ simplices in $\mathcal{N}$, where $\tau_{j}=s_{j-1} \cup s_{j}$ for $j=1, \ldots, m$, and $s_{j} \cap\left(s_{l+1} \backslash s_{l}\right)=\emptyset$ for all $0 \leq j \leq l \leq m-1$. We call this family $\left(s_{0}, \tau_{1}, s_{1}, \ldots, \tau_{m}, s_{m}\right)$ a fence of order 


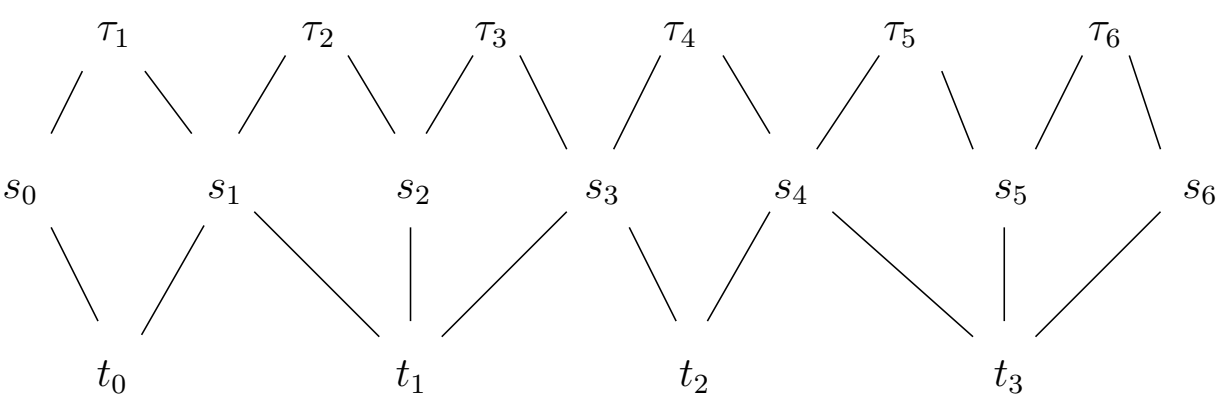

Figure 4 . A $k+1$-fence of length $m=6$ with its $k$-fence. In this example we have $k_{0}=1, k_{1}=2, k_{2}=4, k_{3}=5, J=3$.

$k+1$ and of length $m$. A fence of order $k+1$ and of any length will be called a $k+1$-fence.

The term "fence" is taken from the work [25]. A natural example of a $k+1$ fence is obtained by considering a path $\left(s_{0}, \tau_{1}, s_{1}, \ldots, \tau_{m}, s_{m}\right)$ in the tree $\left(\Gamma^{k}, \Gamma^{k+1}\right)$ connecting $s=s_{0}$ and $s^{\prime}=s_{m}$, where $0 \leq k \leq n-1$ and $\Gamma$ is an $n$-dimensional unoriented Z-system on $\mathcal{N}$. We only need to check that $s_{j} \cap\left(s_{l+1} \backslash s_{l}\right)=\emptyset$ for all $0 \leq j \leq l \leq m-1$. This is obvious if $j=l$ so suppose $j<l$ and consider the paths $\left(s_{j}, \tau_{j+1}, s_{j+1}, \ldots, s_{l-1}, \tau_{l}, s_{l}\right)$ and $\left(s_{j}, \tau_{j+1}, s_{j+1}, \ldots, s_{l}, \tau_{l+1}, s_{l+1}\right)$ in $\left(\Gamma^{k}, \Gamma^{k+1}\right)$. These paths are trees with $l-j$ and $l-j+1$ edges respectively. By the lemma from section 2.6 we see that $\left|s_{j} \cup s_{j+1} \cup \cdots \cup s_{l}\right|=k+1+l-j$ and $\left|s_{j} \cup s_{j+1} \cup \cdots \cup s_{l+1}\right|=k+2+l-j$. Since $\tau_{l+1} \in \Gamma^{k+1}$ is an edge incident on $s_{l}, s_{l+1} \in \Gamma^{k}$, we have that $s_{l} \cap s_{l+1} \in \Gamma^{k-1}$, and hence $\left|s_{l+1} \backslash s_{l}\right|=1$. If the one element of $s_{l+1} \backslash s_{l}$ were in $s_{j}$ then $\left|s_{j} \cup \cdots \cup s_{l} \cup s_{l+1}\right|=k+1+l-j$, which is a contradiction. Hence $s_{j} \cap\left(s_{l+1} \backslash s_{l}\right)=\emptyset$, as claimed.

If $s_{0}, s_{1}, \ldots, s_{m}$ are the $k$-simplices of any $k+1$-fence then by definition $s_{j} \cap$ $\left(s_{l+1} \backslash s_{l}\right)=\emptyset$ for all $0 \leq j \leq l \leq m-1$. We call this the disjointness condition. However a reflected disjointness condition also holds: $\left(s_{j-1} \backslash s_{j}\right) \cap s_{l}=\emptyset$ for all $1 \leq j \leq l \leq m$. The statement is trivial when $l=j$, and when $l=j+1$ we have $\left(s_{j-1} \backslash s_{j}\right) \cap s_{j+1} \subset s_{j-1} \cap\left(s_{j+1} \backslash s_{j}\right)=\emptyset$. The general case is proved by induction on $l$, for if $\left(s_{j-1} \backslash s_{j}\right) \cap s_{l}=\emptyset$ and $a \in\left(s_{j-1} \backslash s_{j}\right) \cap s_{l+1}$ then $a \in s_{j-1} \cap\left(s_{l+1} \backslash s_{l}\right)=\emptyset$, a contradiction.

The following general lemma allows us to construct from a $k+1$-fence another fence of order $k$.

Lemma. Suppose $\left(s_{0}, \tau_{1}, s_{1}, \ldots, \tau_{m}, s_{m}\right)$ is a fence of order $k+1$ and of length $m$. Define $k_{0}=1$ and $k_{j+1}=\min \left\{k_{j}<l \leq m \mid s_{k_{j}-1} \cap s_{k_{j}} \neq s_{l-1} \cap s_{l}\right\}$, for all $j \geq 0$ for which the minimum is over a nonempty set. Let $k_{J}$ be the last one defined in this manner, where $0 \leq J \leq m-1$. Define $t_{j}=s_{k_{j}-1} \cap s_{k_{j}}$ for $j=0,1, \ldots, J$. Then $\left(t_{0}, s_{k_{1}-1}, t_{1}, \ldots, s_{k_{J}-1}, t_{J}\right)$ is a $k$-fence of length $J$ (see Figure 4).

Proof. First we need to check that $t_{0}, t_{1}, \ldots, t_{J}$ are distinct $(k-1)$-simplices. Since $s_{l-1}$ and $s_{l}$ are distinct $k$-simplices contained in the $k+1$ simplex $\tau_{l}$, we see that $s_{l-1} \cap s_{l}$ is a $(k-1)$-simplex of $\mathcal{N}$. Suppose $1 \leq j<l \leq m$ are such that $s_{j-1} \cap s_{j}=$ $s_{l-1} \cap s_{l}$. Then we claim that $s_{j-1} \cap s_{j}=s_{j} \cap s_{j+1}=\cdots=s_{l-2} \cap s_{l-1}=s_{l-1} \cap s_{l}$. To see this, suppose $i \in s_{j-1} \cap s_{j}=s_{l-1} \cap s_{l}$. Since $s_{j-1} \cap\left(s_{l-1} \backslash s_{l-2}\right)=\emptyset$ and 
both $i \in s_{j-1}$ and $i \in s_{l-1}$ it follows that $i \in s_{l-2}$. In a similar way $i$ is a member of all the intervening $s_{k^{\prime}}$. Thus $s_{j-1} \cap s_{j} \subset s_{k^{\prime}-1} \cap s_{k^{\prime}}$ for all $j \leq k^{\prime} \leq l$. Since both sets have $k$ elements, they must be equal as sets. Thus $t_{0}, t_{1}, \ldots, t_{J}$ are distinct $(k-1)$-simplices. Next we show that $t_{j} \cup t_{j+1}=s_{k_{j+1}-1}$ for $j=0,1, \ldots, J-1$. To see this note that by definition $t_{j+1}=s_{k_{j+1}-1} \cap s_{k_{j+1}}$ so clearly $t_{j+1} \subset s_{k_{j+1}-1}$. Also from the definition of $k_{j+1}$ we have $t_{j}=s_{k_{j}-1} \cap s_{k_{j}}=s_{k_{j+1}-2} \cap s_{k_{j+1}-1}$, and hence $t_{j} \subset s_{k_{j+1}-1}$. Thus $t_{j} \cup t_{j+1} \subset s_{k_{j+1}-1}$. Since both $t_{j}$ and $t_{j+1}$ have $k$ elements and are distinct, the union must have at least $k+1$ elements. Thus $t_{j} \cup t_{j+1}=s_{k_{j+1}-1}$ as claimed. Finally we check that $t_{j} \cap\left(t_{l+1} \backslash t_{l}\right)=\emptyset$ for all $0 \leq j \leq l \leq J-1$. Suppose by way of contradiction that $i \in t_{j} \cap\left(t_{l+1} \backslash t_{l}\right)$. Since $k_{l+1} \geq k_{l}+1 \geq k_{j}+1$ we have $i \in s_{k_{j}-1}$ and $i \in s_{k_{l+1}-1}$ but $i \notin s_{k_{l}-1} \cap s_{k_{l}}=s_{k_{l+1}-2} \cap s_{k_{l+1}-1}$. Thus $i \notin s_{k_{l+1}-2}$, and hence $i \in s_{k_{j}-1} \cap\left(s_{k_{l+1}-1} \backslash s_{k_{l+1}-2}\right)$, contradicting the assumption that $\left(s_{0}, \tau_{1}, s_{1}, \ldots, \tau_{m}, s_{m}\right)$ is a $k+1$ fence. Thus if a Z-system contains a $k+1$-fence of length $m$ then it also contains a $k$-fence of length $0 \leq J \leq m-1$ by the above construction.

Remark. For the fence which comes from the path from $s$ to $s^{\prime}$ in the tree $\left(\Gamma^{k}, \Gamma^{k+1}\right)$ associated to an unoriented Z-system, we can use the above lemma to construct the path between $t \subset s$ and $t^{\prime} \subset s^{\prime}$ in the tree $\left(\Gamma^{k-1}, \Gamma^{k}\right)$. If $\left(s_{0}, \tau_{1}, s_{1}, \ldots, \tau_{m}, s_{m}\right)$ is the path in $\left(\Gamma^{k}, \Gamma^{k+1}\right)$ connecting $s=s_{0}$ to $s^{\prime}=s_{m}$, then $\left(t_{0}, s_{k_{1}-1}, t_{1}, \ldots, s_{k_{J}-1}, t_{J}\right)$ will be a path in $\left(\Gamma^{k-1}, \Gamma^{k}\right)$ connecting $t_{0}=s_{0} \cap s_{1}$ to $t_{J}=s_{m-1} \cap s_{m}$. If $t=t_{0}$ and $t^{\prime}=t_{J}$ then we are done. If $t \subset s_{0}, t \in \Gamma^{k-1}$, and $t \neq t_{0}$, then by the reflected disjointness condition $\left(s_{0} \backslash s_{1}\right) \cap s_{j}=\emptyset$ for all $1 \leq j \leq m$ we see that $t$ is distinct from $t_{0}, \ldots, t_{J}, t^{\prime}$. Also $s_{0}$ is an edge incident on $t$ and $t_{0}$. Likewise if $t^{\prime} \subset s_{m}$, $t^{\prime} \in \Gamma^{k-1}$, and $t^{\prime} \neq t_{J}$ then the disjointness condition $s_{j} \cap\left(s_{m} \backslash s_{m-1}\right)=\emptyset$ for all $0 \leq j \leq m-1$ implies that $t^{\prime}$ is distinct from $t, t_{0}, \ldots, t_{J}$. Furthermore $s_{m}$ is an edge incident on $t_{J}$ and $t^{\prime}$. Thus the unique path in $\left(\Gamma^{k-1}, \Gamma^{k}\right)$ connecting $t$ to $t^{\prime}$ passes through the vertices $t_{0}, t_{1}, \ldots, t_{J}$. This result will be useful in sections 3.2 and 3.4 .

\section{Polyspherical Coordinates}

3.1. Internal Coordinates Defined. Although Z-systems are natural combinatorial objects, their true nature is revealed when they are considered as defining polyspherical coordinate systems. Let $\Gamma^{*}$ be an $n$-dimensional Z-system on the set $\mathcal{N}=\{1, \ldots, N\}$, with underlying unoriented Z-system $\Gamma$. Define

$$
\begin{aligned}
\mathcal{B}(\Gamma) & :=\cap\left\{\mathcal{B}_{s} \mid s \in \Gamma^{n-1}\right\} \\
\mathcal{D}_{C}(\Gamma) & :=G_{a} \| \mathcal{B}(\Gamma) .
\end{aligned}
$$

$\mathcal{B}(\Gamma) \subset \mathcal{B}$ depends only on $\Gamma^{n-1} \cdot \mathcal{B}(\Gamma)$ is called the trivialization domain, and $\mathcal{D}_{C}(\Gamma)$ is called the coordinate domain corresponding to the given Z-system. Let $R \in \mathcal{B}(\Gamma)$ be given. Then for every $e=\{i, j\} \in \Gamma^{1}$ we define

$$
L_{e}(R):=\left\|\mathbf{R}_{i}-\mathbf{R}_{j}\right\|
$$

Note that the associated simplex $R_{e}$ must be geometrically independent, and hence $L_{e}(R)>0$.

For any $k=2, \ldots, n$ suppose $e=v_{1} \cup v_{2} \in \Gamma^{k}$ where $v_{1}, v_{2} \in \Gamma^{k-1}$ and $s=$ $v_{1} \cap v_{2} \in \Gamma^{k-2}$. Define $\Pi_{s}$ to be the orthogonal projection onto the subspace of $\mathbb{R}^{n}$ spanned by the set of vectors $\left\{\mathbf{R}_{j}-\mathbf{R}_{i} \mid j \in s \backslash\{i\}\right\}$, where $i \in s$. When $k=2$ so that $s=\{i\}$ we have that $\Pi_{s}$ maps every vector to the zero vector. For 
larger values of $k$ we have that $\Pi_{s}$ does not depend on $i \in s$, as is easy to see. Now suppose $i \in s,\left\{j_{1}\right\}=v_{1} \backslash s$, and $\left\{j_{2}\right\}=v_{2} \backslash s$. Then define

$$
C_{e}(R):=\frac{\left(1-\Pi_{s}\right)\left(\mathbf{R}_{j_{1}}-\mathbf{R}_{i}\right)}{\left\|\left(1-\Pi_{s}\right)\left(\mathbf{R}_{j_{1}}-\mathbf{R}_{i}\right)\right\|} \cdot \frac{\left(1-\Pi_{s}\right)\left(\mathbf{R}_{j_{2}}-\mathbf{R}_{i}\right)}{\left\|\left(1-\Pi_{s}\right)\left(\mathbf{R}_{j_{2}}-\mathbf{R}_{i}\right)\right\|} .
$$

Since the associated $(k-1)$-simplices $R_{v_{1}}$ and $R_{v_{2}}$ must each be contained in a geometrically independent $(n-1)$-simplex, we see that each of the denominators must be positive. However, we must show that each of the unit vectors in the above expression is independent of $i \in s$. To see this suppose $i^{\prime} \neq i, i^{\prime} \in s$. Then $\mathbf{R}_{j}-\mathbf{R}_{i}=\mathbf{R}_{j}-\mathbf{R}_{i^{\prime}}+\left(\mathbf{R}_{i^{\prime}}-\mathbf{R}_{i}\right)$. Since $\Pi_{s}\left(\mathbf{R}_{i^{\prime}}-\mathbf{R}_{i}\right)=\left(\mathbf{R}_{i^{\prime}}-\mathbf{R}_{i}\right)$ we have that $\left(1-\Pi_{s}\right)\left(\mathbf{R}_{j}-\mathbf{R}_{i}\right)=\left(1-\Pi_{s}\right)\left(\mathbf{R}_{j}-\mathbf{R}_{i^{\prime}}\right)$, as desired. If $2 \leq k \leq n-1$ then the associated simplex $R_{e}$ must be geometrically independent, and hence $C_{e}(R) \in$ $(-1,1)$. We will use the coordinate $C_{e}(R)$ rather than the angle $\theta=\cos ^{-1} C_{e}(R)$.

Now suppose $e^{*}=\left[i_{0}, i_{1}, \ldots, i_{n}\right] \in \Gamma_{*}^{n}$, where $e=\left\{i_{0}, i_{1}, \ldots, i_{n}\right\}, s=\left\{i_{0}, i_{1}, \ldots\right.$, $\left.i_{n-2}\right\} \in \Gamma^{n-2}, v_{1}=s \cup\left\{i_{n-1}\right\} \in \Gamma^{n-1}$, and $v_{2}=s \cup\left\{i_{n}\right\} \in \Gamma^{n-1}$. It is always possible to arrange this. $C_{e}(R)$ is defined as above except now we do not know $R_{e}$ is geometrically independent, so we only have that $C_{e}(R) \in[-1,1]$. Since $R_{v_{1}}$ is geometrically independent there is a unique unit vector $\mathbf{e}_{n}$ which is orthogonal to the span of $\left\{\mathbf{R}_{i_{1}}-\mathbf{R}_{i_{0}}, \ldots, \mathbf{R}_{i_{n-1}}-\mathbf{R}_{i_{0}}\right\}$ and such that $\left(\mathbf{R}_{i_{1}}-\mathbf{R}_{i_{0}}, \ldots, \mathbf{R}_{i_{n-1}}-\right.$ $\left.\mathbf{R}_{i_{0}}, \mathbf{e}_{n}\right)$ is positively oriented. We define

$$
S_{e^{*}}(R):=\mathbf{e}_{n} \cdot \frac{\left(1-\Pi_{s}\right)\left(\mathbf{R}_{i_{n}}-\mathbf{R}_{i_{0}}\right)}{\left\|\left(1-\Pi_{s}\right)\left(\mathbf{R}_{i_{n}}-\mathbf{R}_{i_{0}}\right)\right\|} .
$$

Since $R_{v_{2}}$ must be geometrically independent, the denominator in the above expression is positive. Since $R_{s}$ is geometrically independent there exists a unique orthonormal set $\left(\mathbf{e}_{1}, \ldots, \mathbf{e}_{n-2}\right)$ of vectors from $\mathbb{R}^{n}$ and a unique upper triangular $(n-2) \times(n-2)$ matrix $U$ with positive diagonal entries such that $\left(\mathbf{R}_{i_{1}}-\right.$ $\left.\mathbf{R}_{i_{0}}, \ldots, \mathbf{R}_{i_{n-2}}-\mathbf{R}_{i_{0}}\right)=\left(\mathbf{e}_{1}, \ldots, \mathbf{e}_{n-2}\right) U$. This is simply Gram-Schmidt orthogonalization, or QR factorization [34]. The entries of the vectors $\mathbf{e}_{k}$ and of the matrix $U$ are smooth functions of $R$. It follows that $\Pi_{s}=\sum_{k=1}^{n-2} \mathbf{e}_{k} \mathbf{e}_{k}^{T}$. Define

$$
\mathbf{e}_{n-1}=\frac{\left(1-\Pi_{s}\right)\left(\mathbf{R}_{i_{n-1}}-\mathbf{R}_{i_{0}}\right)}{\left\|\left(1-\Pi_{s}\right)\left(\mathbf{R}_{i_{n-1}}-\mathbf{R}_{i_{0}}\right)\right\|}, \quad \text { and } \quad \mathbf{v}=\frac{\left(1-\Pi_{s}\right)\left(\mathbf{R}_{i_{n}}-\mathbf{R}_{i_{0}}\right)}{\left\|\left(1-\Pi_{s}\right)\left(\mathbf{R}_{i_{n}}-\mathbf{R}_{i_{0}}\right)\right\|} \text {. }
$$

It follows that $\left(\mathbf{e}_{1}, \ldots, \mathbf{e}_{n}\right)$ is a positively oriented orthonormal basis of $\mathbb{R}^{n}$. From the definitions above we see that $C_{e}(R)=\mathbf{e}_{n-1} \cdot \mathbf{v}, S_{e^{*}}(R)=\mathbf{e}_{n} \cdot \mathbf{v}$, and hence $\mathbf{v}=C_{s}(R) \mathbf{e}_{n-1}+S_{e^{*}}(R) \mathbf{e}_{n}$. Thus

$$
\operatorname{det}\left(\mathbf{e}_{1}, \ldots, \mathbf{e}_{n-1}, \mathbf{v}\right)=S_{e^{*}}(R) .
$$

This proves that $S_{e^{*}}(R)$ is a smooth function of $R$. See figure 5 for the 3 -dimensional case.

Lemma. The number $S_{e^{*}}(R)$ does not depend on the representative $\left(i_{0}, i_{1}, \ldots, i_{n}\right)$ of the chosen orientational equivalence class $e^{*}$, provided $s=\left\{i_{0}, i_{1}, \ldots, i_{n-2}\right\}$.

Proof. So suppose $\left[i_{0}, i_{1}, \ldots, i_{n}\right]=\left[j_{0}, j_{1}, \ldots, j_{n}\right]$, where $s=\left\{j_{0}, j_{1}, \ldots, j_{n-2}\right\}$. Then $\left\{i_{n-1}, i_{n}\right\}=\left\{j_{n-1}, j_{n}\right\}$, and there are therefore two cases: $\left(i_{n-1}, i_{n}\right)=$ $\left(j_{n-1}, j_{n}\right)$ and $\left(i_{n-1}, i_{n}\right)=\left(j_{n}, j_{n-1}\right)$. In the first case, $\left(i_{n-1}, i_{n}\right)=\left(j_{n-1}, j_{n}\right)$, which cannot happen in a nontrivial way in dimensions 2 and 3 , we see that $\left(j_{0}, j_{1}, \ldots, j_{n-2}\right)$ is an even permutation of $\left(i_{0}, i_{1}, \ldots, i_{n-2}\right)$. Hence $\left(j_{0}, j_{1}, \ldots, j_{n-1}\right)$ is an even permutation of $\left(i_{0}, i_{1}, \ldots, i_{n-1}\right)$. Let $P$ be the $n \times n$ permutation matrix such that $\left(j_{0}, \ldots, j_{n-1}\right)=\left(i_{0}, \ldots, i_{n-1}\right) P$. Let $\mathbf{u}^{T}=(0,1, \ldots, 1) \in\left(\mathbb{R}^{n}\right)^{*}$, and 


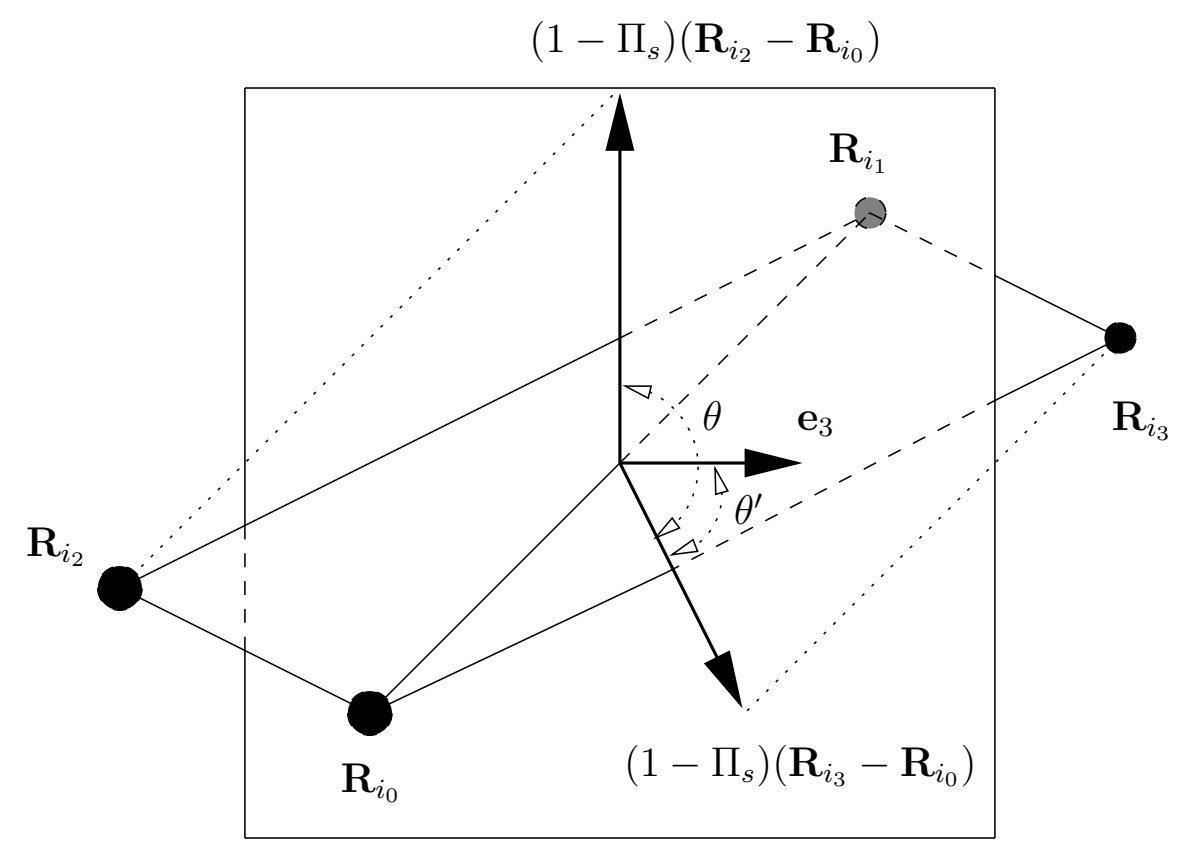

FiguRE 5 . The geometry of wedge angles in dimension $n=3$. In this case $e^{*}=\left[i_{0}, i_{1}, i_{2}, i_{3}\right], s=\left\{i_{0}, i_{1}\right\}, v_{1}=\left\{i_{0}, i_{1}, i_{2}\right\}$, and $v_{2}=\left\{i_{0}, i_{1}, i_{3}\right\}$. The square lies in a plane perpendicular to the line through $\mathbf{R}_{i_{0}}$ and $\mathbf{R}_{i_{1}}$. Both of the vectors $\left(1-\Pi_{s}\right)\left(\mathbf{R}_{i_{2}}-\mathbf{R}_{i_{0}}\right)$ and $\left(1-\Pi_{s}\right)\left(\mathbf{R}_{i_{3}}-\mathbf{R}_{i_{0}}\right)$ lie in this plane since $1-\Pi_{s}$ is an orthogonal projection into this plane. $C_{e}(R)=\cos (\theta)$ and $S_{e^{*}}(R)=\cos \left(\theta^{\prime}\right)=$ $\sin (\theta)>0$ in this picture.

$G=I-\hat{\mathbf{e}}_{1} \mathbf{u}^{T}$ an $n \times n$ Gauss transformation; note that $G^{-1}=I+\hat{\mathbf{e}}_{1} \mathbf{u}^{T}$. Then we have that

$$
\begin{aligned}
&\left(\begin{array}{ccccc}
1 & 0 & \ldots & 0 & 0 \\
\mathbf{R}_{j_{0}} & \mathbf{R}_{j_{1}}-\mathbf{R}_{j_{0}} & \ldots & \mathbf{R}_{j_{n-1}}-\mathbf{R}_{j_{0}} & \mathbf{e}_{n}
\end{array}\right) \\
& \quad=\left(\begin{array}{ccccc}
1 & 1 & \ldots & 1 & 0 \\
\mathbf{R}_{j_{0}} & \mathbf{R}_{j_{1}} & \ldots & \mathbf{R}_{j_{n-1}} & \mathbf{e}_{n}
\end{array}\right)\left(\begin{array}{cc}
G & \boldsymbol{\theta} \\
\boldsymbol{\theta}^{T} & 1
\end{array}\right) \\
&=\left(\begin{array}{ccccc}
1 & 1 & \ldots & 1 & 0 \\
\mathbf{R}_{i_{0}} & \mathbf{R}_{i_{1}} & \ldots & \mathbf{R}_{i_{n-1}} & \mathbf{e}_{n}
\end{array}\right)\left(\begin{array}{cc}
P & \boldsymbol{\theta} \\
\boldsymbol{\theta}^{T} & 1
\end{array}\right)\left(\begin{array}{cc}
G & \boldsymbol{\theta} \\
\boldsymbol{\theta}^{T} & 1
\end{array}\right) \\
&=\left(\begin{array}{ccccc}
1 & 0 & \ldots & 0 & 0 \\
\mathbf{R}_{i_{0}} & \mathbf{R}_{i_{1}}-\mathbf{R}_{i_{0}} & \ldots & \mathbf{R}_{i_{n-1}}-\mathbf{R}_{i_{0}} & \mathbf{e}_{n}
\end{array}\right)\left(\begin{array}{cc}
G^{-1} & \boldsymbol{\theta} \\
\boldsymbol{\theta}^{T} & 1
\end{array}\right)\left(\begin{array}{cc}
P G & \boldsymbol{\theta} \\
\boldsymbol{\theta}^{T} & 1
\end{array}\right) .
\end{aligned}
$$

Since $\operatorname{det}\left(G^{-1} P G\right)=\operatorname{det}(P)=1$ we see that

$$
\operatorname{det}\left(\mathbf{R}_{j_{1}}-\mathbf{R}_{j_{0}}, \ldots, \mathbf{R}_{j_{n-1}}-\mathbf{R}_{j_{0}}, \mathbf{e}_{n}\right)=\operatorname{det}\left(\mathbf{R}_{i_{1}}-\mathbf{R}_{i_{0}}, \ldots, \mathbf{R}_{i_{n-1}}-\mathbf{R}_{i_{0}}, \mathbf{e}_{n}\right)>0 .
$$

Also $\hat{\mathbf{e}}_{1}^{T}\left(I+\hat{\mathbf{e}}_{1} \mathbf{u}^{T}\right) P\left(I-\hat{\mathbf{e}}_{1} \mathbf{u}^{T}\right)=\left(\hat{\mathbf{e}}_{1}^{T}+\mathbf{u}^{T}\right) P\left(I-\hat{\mathbf{e}}_{1} \mathbf{u}^{T}\right)=\left(\hat{\mathbf{e}}_{1}^{T}+\mathbf{u}^{T}\right)\left(I-\hat{\mathbf{e}}_{1} \mathbf{u}^{T}\right)=$ $\hat{\mathbf{e}}_{1}^{T}+\mathbf{u}^{T}-\left(\hat{\mathbf{e}}_{1}^{T} \hat{\mathbf{e}}_{1}\right) \mathbf{u}^{T}-\left(\mathbf{u}^{T} \hat{\mathbf{e}}_{1}\right) \mathbf{u}^{T}=\hat{\mathbf{e}}_{1}^{T}$. Therefore $G^{-1} P G=\left(\begin{array}{cc}1 & \boldsymbol{\theta}^{T} \\ \mathbf{v} & H\end{array}\right)$, where $H$ 
is an $(n-1) \times(n-1)$ matrix with unit determinant. Since

$$
\left(\mathbf{R}_{j_{0}}, \mathbf{R}_{j_{1}}-\mathbf{R}_{j_{0}}, \ldots, \mathbf{R}_{j_{n-1}}-\mathbf{R}_{j_{0}}\right)=\left(\mathbf{R}_{i_{0}}, \mathbf{R}_{i_{1}}-\mathbf{R}_{i_{0}}, \ldots, \mathbf{R}_{i_{n-1}}-\mathbf{R}_{i_{0}}\right)\left(\begin{array}{cc}
1 & \boldsymbol{\theta}^{T} \\
\mathbf{v} & H
\end{array}\right)
$$

we see that

$$
\left(\mathbf{R}_{j_{1}}-\mathbf{R}_{j_{0}}, \ldots, \mathbf{R}_{j_{n-1}}-\mathbf{R}_{j_{0}}\right)=\left(\mathbf{R}_{i_{1}}-\mathbf{R}_{i_{0}}, \ldots, \mathbf{R}_{i_{n-1}}-\mathbf{R}_{i_{0}}\right) H
$$

and hence the same vector $\mathbf{e}_{n}$ works for both representatives of the orientational equivalence class. Since we have already seen that $\left(1-\Pi_{s}\right)\left(\mathbf{R}_{j_{n}}-\mathbf{R}_{j_{0}}\right)=(1-$ $\left.\Pi_{s}\right)\left(\mathbf{R}_{i_{n}}-\mathbf{R}_{i_{0}}\right)$, we have that $S_{e^{*}}(R)$ is independent of the representative of the orientational equivalence class in this case.

Now consider the case where $\left(i_{n-1}, i_{n}\right)=\left(j_{n}, j_{n-1}\right)$. Therefore $\left(j_{0}, j_{1}, \ldots, j_{n-2}\right)$ is an odd permutation of $\left(i_{0}, i_{1}, \ldots, i_{n-2}\right)$. Let $B=\left(\mathbf{R}_{i_{1}}-\mathbf{R}_{i_{0}}, \ldots, \mathbf{R}_{i_{n-2}}-\mathbf{R}_{i_{0}}\right)$. Using an argument similar to that given in the first case we have that there is an $(n-$ $2) \times(n-2)$ matrix $K$ such that $\operatorname{det} K=-1$ and $\left(\mathbf{R}_{j_{1}}-\mathbf{R}_{j_{0}}, \ldots, \mathbf{R}_{j_{n-2}}-\mathbf{R}_{j_{0}}\right)=B K$. There exists a vector $\mathbf{z} \in \mathbb{R}^{n-1}$ such that $\mathbf{R}_{i_{0}}-\mathbf{R}_{j_{0}}=B \mathbf{z}$. Therefore $\mathbf{R}_{j_{n-1}}-\mathbf{R}_{j_{0}}=$ $\mathbf{R}_{i_{n}}-\mathbf{R}_{i_{0}}+B \mathbf{z}$ and $\mathbf{R}_{j_{n}}-\mathbf{R}_{j_{0}}=\mathbf{R}_{i_{n-1}}-\mathbf{R}_{i_{0}}+B \mathbf{z}$. Consequently $\left(1-\Pi_{s}\right)\left(\mathbf{R}_{j_{n-1}}-\right.$ $\left.\mathbf{R}_{j_{0}}\right)=\left(1-\Pi_{s}\right)\left(\mathbf{R}_{i_{n}}-\mathbf{R}_{i_{0}}\right)$ and $\left(1-\Pi_{s}\right)\left(\mathbf{R}_{j_{n}}-\mathbf{R}_{j_{0}}\right)=\left(1-\Pi_{s}\right)\left(\mathbf{R}_{i_{n-1}}-\mathbf{R}_{i_{0}}\right)$, and hence $\tilde{\mathbf{e}}_{n-1}=\mathbf{v}$, and $\tilde{\mathbf{v}}=\mathbf{e}_{n-1}$. Since $\tilde{\mathbf{e}}_{n-1}=\mathbf{v}=C_{s}(R) \mathbf{e}_{n-1}+S_{e^{*}}(R) \mathbf{e}_{n}$ it is reasonable to define $\tilde{\mathbf{e}}_{n}=S_{e^{*}}(R) \mathbf{e}_{n-1}-C_{e}(R) \mathbf{e}_{n}$, since then we would have $\tilde{\mathbf{e}}_{n-1} \cdot \tilde{\mathbf{e}}_{n}=0$ and $\tilde{\mathbf{e}}_{n} \cdot \tilde{\mathbf{v}}=\left[S_{e^{*}}(R) \mathbf{e}_{n-1}-C_{e}(R) \mathbf{e}_{n}\right] \cdot \mathbf{e}_{n-1}=S_{e^{*}}(R)$ as desired. Since $\tilde{\mathbf{e}}_{n} \cdot \tilde{\mathbf{e}}_{n}=S_{e^{*}}(R)^{2}+C_{e}(R)^{2}=\mathbf{v} \cdot \mathbf{v}=1$ we see that $\tilde{\mathbf{e}}_{n}$ is a unit vector. To finish showing that this choice for $\tilde{\mathbf{e}}_{n}$ has all the properties specified for it in the definition, we compute

$$
\begin{aligned}
\left(\mathbf{R}_{j_{1}}-\mathbf{R}_{j_{0}}, \ldots, \mathbf{R}_{j_{n-1}}-\mathbf{R}_{j_{0}}, \tilde{\mathbf{e}}_{n}\right) & \\
& =\left(\mathbf{R}_{i_{1}}-\mathbf{R}_{i_{0}}, \ldots, \mathbf{R}_{i_{n-2}}-\mathbf{R}_{i_{0}}, \mathbf{R}_{j_{n-1}}-\mathbf{R}_{j_{0}}, \tilde{\mathbf{e}}_{n}\right)\left(\begin{array}{ccc}
K & \boldsymbol{\theta} & \boldsymbol{\theta} \\
\boldsymbol{\theta}^{T} & 1 & 0 \\
\boldsymbol{\theta}^{T} & 0 & 1
\end{array}\right) \\
& =\left(\mathbf{e}_{1}, \ldots, \mathbf{e}_{n-2}, \mathbf{R}_{j_{n-1}}-\mathbf{R}_{j_{0}}, \tilde{\mathbf{e}}_{n}\right)\left(\begin{array}{ccc}
U & \boldsymbol{\theta} & \boldsymbol{\theta} \\
\boldsymbol{\theta}^{T} & 1 & 0 \\
\boldsymbol{\theta}^{T} & 0 & 1
\end{array}\right)\left(\begin{array}{ccc}
K & \boldsymbol{\theta} & \boldsymbol{\theta} \\
\boldsymbol{\theta}^{T} & 1 & 0 \\
\boldsymbol{\theta}^{T} & 0 & 1
\end{array}\right) \\
& =\left(\mathbf{e}_{1}, \ldots, \mathbf{e}_{n-2}, \tilde{\mathbf{e}}_{n-1}, \tilde{\mathbf{e}}_{n}\right)\left(\begin{array}{ccc}
I & \mathbf{y} & \boldsymbol{\theta} \\
\boldsymbol{\theta}^{T} & l & 0 \\
\boldsymbol{\theta}^{T} & 0 & 1
\end{array}\right)\left(\begin{array}{ccc}
U K & \boldsymbol{\theta} & \boldsymbol{\theta} \\
\boldsymbol{\theta}^{T} & 1 & 0 \\
\boldsymbol{\theta}^{T} & 0 & 1
\end{array}\right) \\
& =\left(\mathbf{e}_{1}, \ldots, \mathbf{e}_{n-2}, \mathbf{e}_{n-1}, \mathbf{e}_{n}\right)\left(\begin{array}{ccc}
I & \boldsymbol{\theta} & \boldsymbol{\theta} \\
\boldsymbol{\theta}^{T} & C_{e}(R) & S_{e^{*}}(R) \\
\boldsymbol{\theta}^{T} & S_{e^{*}}(R) & -C_{e}(R)
\end{array}\right)\left(\begin{array}{ccc}
U K & \mathbf{y} & \boldsymbol{\theta} \\
\boldsymbol{\theta}^{T} & l & 0 \\
\boldsymbol{\theta}^{T} & 0 & 1
\end{array}\right),
\end{aligned}
$$

where $\mathbf{x}=\mathbf{R}_{j_{n-1}}-\mathbf{R}_{j_{0}}, \mathbf{y}=\left(\mathbf{e}_{1}, \ldots, \mathbf{e}_{n-2}\right)^{T} \mathbf{x}$, and $l=\left\|\left(1-\Pi_{s}\right) \mathbf{x}\right\|$. Thus $\left(\mathbf{R}_{j_{1}}-\right.$ $\left.\mathbf{R}_{j_{0}}, \ldots, \mathbf{R}_{j_{n-1}}-\mathbf{R}_{j_{0}}, \tilde{\mathbf{e}}_{n}\right)$ is positively oriented.

Thus we define $Z_{e^{*}}(R):=C_{e}(R)+i S_{e^{*}}(R) \in S^{1}=\{z \in \mathbb{C}|| z \mid=1\}$. Clearly if $e^{*}$ is replaced by the opposite orientational equivalence class $-e^{*}$ with the same underlying $n$-simplex $e$ then $S_{-e^{*}}(R)=-S_{e^{*}}(R)$. We will use the coordinate $Z_{e^{*}}(R)$ rather than choosing a branch for the angle $\varphi$, where $Z_{e^{*}}(R)=e^{i \varphi}$. We consider $S^{1}$ to be a one dimensional real manifold. 
We define the parameter domain $\mathcal{D}_{P}\left(\Gamma^{*}\right)$ to be

$$
\mathcal{D}_{P}\left(\Gamma^{*}\right):=(0, \infty)^{\Gamma^{1}} \times\left[\prod_{k=2}^{n-1}(-1,1)^{\Gamma^{k}}\right] \times\left(S^{1}\right)^{\Gamma^{n}} .
$$

When $R \in \mathcal{B}(\Gamma)$ is given then, taking the values at $R$ of all the coordinate functions we have defined together, an element of $\mathcal{D}_{P}\left(\Gamma^{*}\right)$, also called a parameter specification, is defined:

$$
\left(e \in \Gamma^{1} \mapsto L_{e}(R),\left\{e \in \Gamma^{k} \mapsto C_{e}(R)\right\}_{k=2}^{n-1}, e^{*} \in \Gamma_{*}^{n} \mapsto Z_{e^{*}}(R)\right) .
$$

Thus we can think of elements of $\mathcal{D}_{P}\left(\Gamma^{*}\right)$ as being a labeling of each of the edges of each of the trees of the given Z-system $\Gamma^{*}$ with appropriate numerical coordinate values. Thus a pair $\left(\Gamma^{*}, \gamma\right)$ consisting of a Z-system and an element $\gamma$ of $\mathcal{D}_{P}\left(\Gamma^{*}\right)$ will be called a labeled Z-system.

3.2. Sites and Conforming Poses. Suppose $r=\left(i_{0}, \ldots, i_{n-1}\right) \in \mathcal{N}^{n}$ is such that $\left|\left\{i_{0}, \ldots, i_{n-1}\right\}\right|=n$. We call $r$ a site. $i_{k}$ is called the $k$ th element of the site $r$, $k=0,1, \ldots, n-1$. Let $s_{k}(r):=\left\{i_{0}, \ldots, i_{k}\right\}$ for all $0 \leq k \leq n-1$. The set of abstract simplices $\left\{s_{0}(r), \ldots, s_{n-1}(r)\right\}$ is said to be the flag associated to the site $r$; a site and its flag contain exactly the same information, but in different forms. Given a site $r$ and $R \in \mathcal{B}_{s_{n-1}(r)}$ we can define the pose at the site $r$ conforming to the configuration $R$ as follows:

$$
\begin{aligned}
\mathbf{e}_{0}= & \mathbf{R}_{i_{0}}, \quad \mathbf{e}_{1}=\frac{\mathbf{R}_{i_{1}}-\mathbf{R}_{i_{0}}}{\left\|\mathbf{R}_{i_{1}}-\mathbf{R}_{i_{0}}\right\|}, \quad \mathbf{e}_{2}=\frac{\left(1-\mathbf{e}_{1} \mathbf{e}_{1}^{T}\right)\left(\mathbf{R}_{i_{2}}-\mathbf{R}_{i_{0}}\right)}{\left\|\left(1-\mathbf{e}_{1} \mathbf{e}_{1}^{T}\right)\left(\mathbf{R}_{i_{2}}-\mathbf{R}_{i_{0}}\right)\right\|}, \ldots, \\
\mathbf{e}_{n-1}= & \frac{\left(1-\sum_{k=1}^{n-2} \mathbf{e}_{k} \mathbf{e}_{k}^{T}\right)\left(\mathbf{R}_{i_{n-1}}-\mathbf{R}_{i_{0}}\right)}{\left\|\left(1-\sum_{k=1}^{n-2} \mathbf{e}_{k} \mathbf{e}_{k}^{T}\right)\left(\mathbf{R}_{i_{n-1}}-\mathbf{R}_{i_{0}}\right)\right\|}, \\
\mathbf{e}_{n}= & \text { the unique unit vector in } \mathbb{R}^{n} \text { perpendicular to } \mathbf{e}_{1}, \ldots, \mathbf{e}_{n-1} \text { such that } \\
& \left(\mathbf{e}_{1}, \ldots, \mathbf{e}_{n}\right) \text { is positively oriented. }
\end{aligned}
$$

Each of these vectors is well-defined since the simplex $R_{s_{n-1}(r)}$ is geometrically independent for $R \in \mathcal{B}_{s_{n-1}(r)}$. Let $E_{r}(R):=\left(\mathbf{e}_{0}, \mathbf{e}_{1}, \ldots, \mathbf{e}_{n}\right) \in \mathcal{P}$ denote this pose. Notice that $\Pi_{s_{j}(r)}=\sum_{k=1}^{j-1} \mathbf{e}_{k} \mathbf{e}_{k}^{T}$, for $j=1, \ldots, n-1$. If $(\mathbf{b}, A) \in G_{a}$ then it is clear that $E_{r}((\mathbf{b}, A) \cdot R)=(\mathbf{b}, A) E_{r}(R)$. The following diagram commutes:

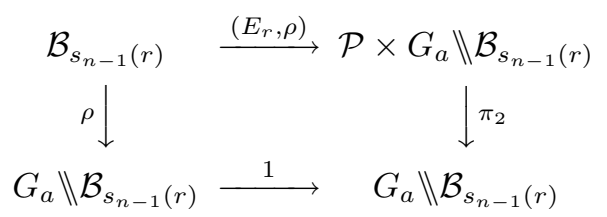

This shows that choice of a site $r$ determines a local trivialization in the principal bundle $\rho: \mathcal{B} \rightarrow G_{a} \backslash \mathcal{B}$. (Here we identify $G_{a}$ and $\mathcal{P}$ in the obvious way.)

- Let vert $\mathcal{S}(\Gamma)$ denote the set of all sites $r$ such that $s_{k}(r) \in \Gamma^{k}$ for $0 \leq k \leq n-1$. Such sites are said to be associated with or from the unoriented Z-system $\Gamma$. Note that vert $\mathcal{S}(\Gamma)$ does not depend on the choice of $\Gamma^{n}$.

- For $1 \leq k \leq n-1$ let edge $_{k} \mathcal{S}(\Gamma)$ denote the set of all two element subsets $\left\{\left(i_{0}, \ldots, i_{n-1}\right),\left(\overline{j_{0}}, \ldots, j_{n-1}\right)\right\}$ of vert $\mathcal{S}(\Gamma)$ such that $\left(j_{0}, \ldots, j_{n-1}\right)$ can be obtained from $\left(i_{0}, \ldots, i_{n-1}\right)$ by transposition of elements $i_{k-1}$ and $i_{k}$. 
- If $\Gamma^{*}$ is a Z-system, with underlying unoriented Z-system $\Gamma$, then let edge ${ }_{n} \mathcal{S}\left(\Gamma^{*}\right)$ (resp. edge ${ }_{n} \mathcal{S}(\Gamma)$ ) denote the set of all ordered pairs

$$
\left(\left(i_{0}, \ldots, i_{n-2}, i\right),\left(i_{0}, \ldots, i_{n-2}, i^{\prime}\right)\right)
$$

of distinct elements of vert $\mathcal{S}(\Gamma)$ (resp. two element subsets

$$
\left\{\left(i_{0}, \ldots, i_{n-2}, i\right),\left(i_{0}, \ldots, i_{n-2}, i^{\prime}\right)\right\}
$$

of vert $\mathcal{S}(\Gamma))$ such that $\left[i_{0}, \ldots, i_{n-2}, i, i^{\prime}\right] \in \Gamma_{*}^{n}\left(\right.$ resp. $\left.\left\{i_{0}, \ldots, i_{n-2}, i, i^{\prime}\right\} \in \Gamma^{n}\right)$.

- Define edge $\mathcal{S}(\Gamma):=\cup_{k=1}^{n} \operatorname{edge}_{k} \mathcal{S}(\Gamma)$.

- The data (vert $\mathcal{S}(\Gamma)$, edge $\mathcal{S}(\Gamma)$ ) forms a traditional graph called the undirected site graph $\mathcal{S}(\Gamma)$. If $\left\{r_{1}, r_{2}\right\}$ is an edge of the undirected site graph, then the associated pair of flags $\left\{s_{0}\left(r_{1}\right), \ldots, s_{n-1}\left(r_{1}\right)\right\},\left\{s_{0}\left(r_{2}\right), \ldots, s_{n-1}\left(r_{2}\right)\right\}$ has the property that for some $0 \leq k \leq n-1$ we have $s_{k}\left(r_{1}\right) \neq s_{k}\left(r_{2}\right)$ and $s_{j}\left(r_{1}\right)=s_{j}\left(r_{2}\right)$ for all $0 \leq j \leq n-1, j \neq k$. This uniform condition on the flags shows that the set of edges of the undirected site graph is naturally defined.

- Define edge $\mathcal{S}\left(\Gamma^{*}\right):=\left[\cup_{k=1}^{n-1} \operatorname{edge}_{k} \mathcal{S}(\Gamma)\right] \cup \operatorname{edge}_{n} \mathcal{S}\left(\Gamma^{*}\right)$.

- The data (vert $\mathcal{S}(\Gamma)$, edge $\mathcal{S}\left(\Gamma^{*}\right)$ ) forms a traditional graph with certain edges being directed, called the site graph $\mathcal{S}\left(\Gamma^{*}\right)$.

- Let $\mathcal{G}(\Gamma)$ be the set of all ordered pairs $\left(\left(i_{0}, \ldots, i_{n-2}, i\right),\left(i_{0}, \ldots, i_{n-2}, i^{\prime}\right)\right)$ of sites in vert $\mathcal{S}(\Gamma)$. Let this set $\mathcal{G}(\Gamma)$ be equipped with the following partially defined binary operation: $\left(r_{1}, r_{2}\right)\left(r_{2}, r_{3}\right)=\left(r_{1}, r_{3}\right)$. Thus the operation $\left(r_{1}, r_{2}\right)\left(r_{3}, r_{4}\right)$ is undefined unless $r_{2}=r_{3}$. With this definition, $\mathcal{G}(\Gamma)$ becomes what is called a pair groupoid.

- The pair (vert $\mathcal{S}(\Gamma),\left[\cup_{k=1}^{n-1} \operatorname{edge}_{k} \mathcal{S}(\Gamma)\right] \cup \mathcal{G}(\Gamma)$ ) is called the site network of $\Gamma$ (see Figure 6). Note that the site network does not depend on the choice of $\Gamma_{*}^{n}$ or even of $\Gamma^{n}$.

Lemma. If $\Gamma$ is an $n$-dimensional unoriented $Z$-system on $\mathcal{N}$ then the undirected site graph $\mathcal{S}(\Gamma)$ is connected.

Proof. Suppose $r, r^{\prime}$ are distinct sites in vert $\mathcal{S}(\Gamma)$. We must construct a sequence $r=r_{0}, r_{1}, \ldots, r_{m}=r^{\prime}$ of distinct sites in vert $\mathcal{S}(\Gamma)$ such that for all $k=1, \ldots, m$ the pair $\left\{r_{k-1}, r_{k}\right\} \in$ edge $\mathcal{S}(\Gamma)$. This represents a path from $r$ to $r^{\prime}$ in the undirected site graph. Note that there is a unique path in the tree $\left(\Gamma^{k}, \Gamma^{k+1}\right)$ connecting $s_{k}(r)$ to $s_{k}\left(r^{\prime}\right), k=0,1, \ldots, n-1$. The remark after the lemma of section 2.7 shows how these paths relate to one another.

Suppose the sites $r_{0}, \ldots, r_{j} \in \operatorname{vert} \mathcal{S}(\Gamma)$ have been chosen, $j \geq 0$, and $s_{k}\left(r_{j}\right)$ is on the path in $\left(\Gamma^{k}, \Gamma^{k+1}\right)$ connecting $s_{k}(r)$ to $s_{k}\left(r^{\prime}\right), 0 \leq k \leq n-1$. Let $U=\left\{k \mid 0 \leq k \leq n-1, s_{k}\left(r_{j}\right) \neq s_{k}\left(r^{\prime}\right)\right\}$, and for $k \in U$ let $s_{k}^{\prime} \in \Gamma^{k}$ be the next vertex on the path in $\left(\Gamma^{k}, \Gamma^{k+1}\right)$ connecting $s_{k}(r)$ to $s_{k}\left(r^{\prime}\right)$ after $s_{k}\left(r_{j}\right)$. Let $D=\cup_{k \in U}\{k, k+1\}$ and define $\iota: D \rightarrow\{0,1\}$ by the rule:

$$
\iota(k)= \begin{cases}0 & k \in U, s_{k-1}\left(r_{j}\right) \subset s_{k}^{\prime}, \\ 1 & k-1 \in U, s_{k-1}^{\prime} \subset s_{k}\left(r_{j}\right)\end{cases}
$$

In conjunction with the above we define $s_{-1}\left(r_{j}\right)=\emptyset$ and $s_{n}\left(r_{j}\right)=s_{n-1}\left(r_{j}\right) \cup s_{n-1}^{\prime}$ when $n-1 \in U$. To insure that $\iota(k)$ is defined for every $k \in D$ suppose $k \in U$ but $k-1 \notin U$. Then $s_{k-1}\left(r_{j}\right)=s_{k-1}\left(r^{\prime}\right)$ and the remark of section 2.7 implies that $s_{k-1}\left(r_{j}\right) \subset s_{k}^{\prime}$ and hence $\iota(k)=0$. Likewise suppose $k \notin U$ and $k-1 \in U$. Then $s_{k}\left(r_{j}\right)=s_{k}\left(r^{\prime}\right)$ and the remark of section 2.7 implies that $s_{k-1}^{\prime} \subset s_{k}\left(r_{j}\right)$ and hence $\iota(k)=1$. To insure that $\iota(k)$ is well-defined, suppose $k-1, k \in U$. From the remark 


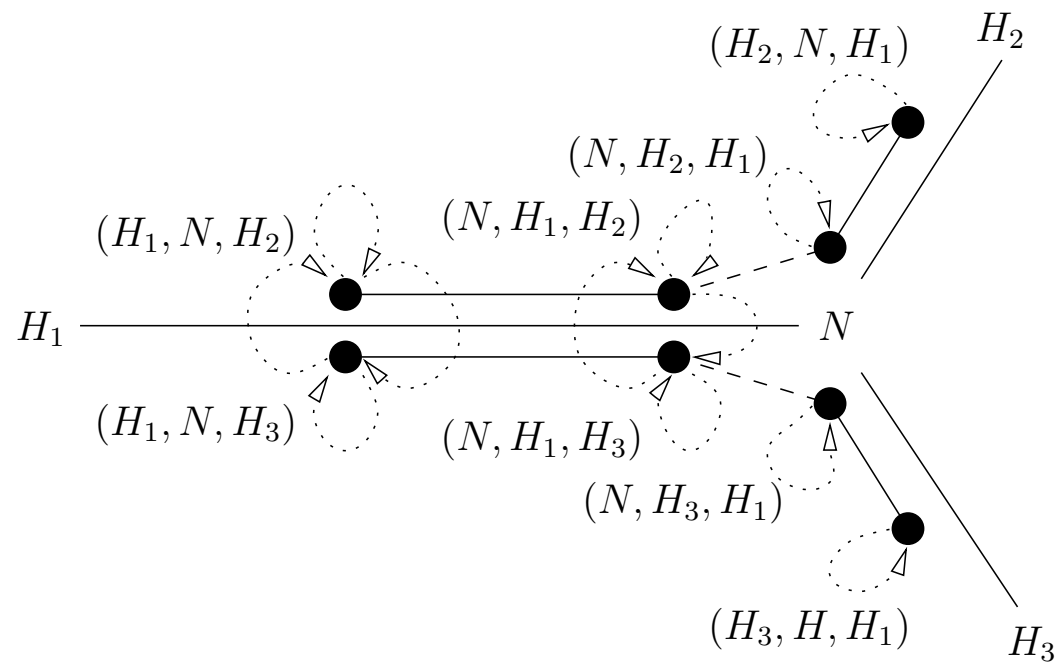

Figure 6 . The site network for ammonia, superimposed on the molecular graph for the molecule. The Z-system has $\mathcal{N}=\left\{N, H_{1}\right.$, $\left.H_{2}, H_{3}\right\}, \Gamma^{1}=\left\{\left\{N, H_{1}\right\},\left\{N, H_{2}\right\},\left\{N, H_{3}\right\}\right\}, \Gamma^{2}=\left\{\left\{H_{1}, N, H_{2}\right\}\right.$, $\left.\left\{H_{1}, N, H_{3}\right\}\right\}$, and $\Gamma_{*}^{3}=\left\{\left[N, H_{1}, H_{2}, H_{3}\right]\right\}$. Sites are indicated by dark circles, and labeled with the corresponding ordered triples of atom names. Edges in the site graph are indicated by solid and dashed lines. Elements of the pair groupoid are indicated by dotted curving arrows.

of section 2.7 we see that $s_{k}\left(r_{j}\right) \cap s_{k}^{\prime}$ is either $s_{k-1}\left(r_{j}\right)$ or $s_{k-1}^{\prime}$, but not both. In the first case we have $\iota(k)=0$ and in the second case $\iota(k)=1$.

If $U=\emptyset$ then $r_{j}=r^{\prime}$ and we are done. Otherwise suppose $k_{1} \leq k_{2}$ and $k_{2}-k_{1}$ is as large as possible subject to the condition that $k \in U$ for all $k_{1} \leq k \leq k_{2}$. Then $k \in D$ for all $k_{1} \leq k \leq k_{2}+1$ and $\iota\left(k_{1}\right)=0$ and $\iota\left(k_{2}+1\right)=1$. Then there exists an integer $k^{*}$ such that $k_{1} \leq k^{*} \leq k_{2}, \iota\left(k^{*}\right)=0$, and $\iota\left(k^{*}+1\right)=1$. Thus $k^{*} \in U$, $s_{k^{*}-1}\left(r_{j}\right) \subset s_{k^{*}}^{\prime}$, and $s_{k^{*}}^{\prime} \subset s_{k^{*}+1}\left(r_{j}\right)$. Now define the site $r_{j+1}$, whose flag satisfies

$$
s_{k}\left(r_{j+1}\right)= \begin{cases}s_{k}\left(r_{j}\right) & 0 \leq k \leq n-1, k \neq k^{*}, \\ s_{k^{*}}^{\prime} & k=k^{*}\end{cases}
$$

Clearly $r_{j+1}$ is a site associated with $\Gamma$ and $\left\{r_{j}, r_{j+1}\right\}$ is an edge of the undirected site graph $\mathcal{S}(\Gamma)$. Clearly $s_{k}\left(r_{j+1}\right)$ is on the path in $\left(\Gamma^{k}, \Gamma^{k+1}\right)$ connecting $s_{k}(r)$ to $s_{k}\left(r^{\prime}\right), 0 \leq k \leq n-1$.

Thus we have given an inductive construction of a path in the undirected site graph connecting $r$ to $r^{\prime}$. This construction must terminate once all the paths in $\left(\Gamma^{k}, \Gamma^{k+1}\right)$ connecting $s_{k}(r)$ to $s_{k}\left(r^{\prime}\right), 0 \leq k \leq n-1$, have been traversed.

3.3. Coordinate Transformations. The site graph associated to a Z-system may seem to be an unnecessary construct, but it greatly illuminates the features of the coordinates we have defined. To see this, define the following $(n+1) \times(n+1)$ 
matrix-valued functions:

$$
\begin{aligned}
T_{1}(L)= & \left(\begin{array}{cccc}
1 & 0 & \boldsymbol{\theta}^{T} & 0 \\
L & -1 & \boldsymbol{\theta}^{T} & 0 \\
\boldsymbol{\theta} & \boldsymbol{\theta} & I & \boldsymbol{\theta} \\
0 & 0 & \boldsymbol{\theta}^{T} & -1
\end{array}\right), \quad L>0 ; \\
T_{k}(C)= & \left(\begin{array}{ccccc}
I_{1} & \boldsymbol{\theta}_{1} & \boldsymbol{\theta}_{1} & \Theta & \boldsymbol{\theta}_{1} \\
\boldsymbol{\theta}_{1}^{T} & C & S & \boldsymbol{\theta}_{2}^{T} & 0 \\
\boldsymbol{\theta}_{1}^{T} & S & -C & \boldsymbol{\theta}_{2}^{T} & 0 \\
\Theta^{T} & \boldsymbol{\theta}_{2} & \boldsymbol{\theta}_{2} & I_{2} & \boldsymbol{\theta}_{2} \\
\boldsymbol{\theta}_{1}^{T} & 0 & 0 & \boldsymbol{\theta}_{2}^{T} & -1
\end{array}\right),
\end{aligned}
$$

where $I_{1}$ is a $(k-1) \times(k-1)$ identity matrix,

for $C \in(-1,1), S=\sqrt{1-C^{2}}, 2 \leq k \leq n-1$;

$$
T_{n}(Z)=\left(\begin{array}{ccc}
I & \boldsymbol{\theta} & \boldsymbol{\theta} \\
\boldsymbol{\theta}^{T} & C & -S \\
\boldsymbol{\theta}^{T} & S & C
\end{array}\right), \quad Z=C+i S \in S^{1} .
$$

Note that each of the mappings $T_{k}$ takes values in $G_{p}$ and is injective for all $1 \leq k \leq$ $n$, and $T_{1}(L)^{-1}=T_{1}(L)$ and $T_{k}(C)^{-1}=T_{k}(C)$ whenever $2 \leq k<n$. The mapping $T_{n}: S^{1} \rightarrow G_{p}$ is a group homomorphism, so that $T_{n}(Z)^{-1}=T_{n}(\bar{Z})$. Note that for $n \geq 3$ we also have the identity: $T_{n}(Z) T_{1}(L)=T_{1}(L) T_{n}(\bar{Z})=\left[T_{n}(Z) T_{1}(L)\right]^{-1}$.

Now suppose $R \in \mathcal{B}(\Gamma)$ is given. To each site $r \in$ vert $\mathcal{S}(\Gamma)$ we assign the pose $E_{r}(R)$ at that site. Thus the vertices of the site graph become labeled with poses. Now for every edge $\left\{r, r^{\prime}\right\} \in\left[\cup_{k=1}^{n-1} \operatorname{edge}_{k} \mathcal{S}(\Gamma)\right]$ or $\left(r, r^{\prime}\right) \in \operatorname{edge}_{n} \mathcal{S}\left(\Gamma^{*}\right)$ of the site graph, there is a unique matrix $\mathcal{A}_{r, r^{\prime}}(R) \in G_{p}$ such that $E_{r}(R) \mathcal{A}_{r, r^{\prime}}(R)=E_{r^{\prime}}(R)$. Because of the special structure of the poses this matrix always turns out to be one of the above three types.

Theorem. Suppose $\Gamma^{*}$ is a Z-system and $R \in \mathcal{B}(\Gamma)$. Suppose $r, r^{\prime}$ are sites of $\Gamma$. Let $\mathcal{A}_{r, r^{\prime}}(R)$ denote the unique matrix in $G_{p}$ such that $E_{r}(R) \mathcal{A}_{r, r^{\prime}}(R)=E_{r^{\prime}}(R)$.

(1) If $\left\{r, r^{\prime}\right\} \in$ edge $_{1} \mathcal{S}(\Gamma)$ and $e=s_{1}(r)=s_{1}\left(r^{\prime}\right)$ then $\mathcal{A}_{r, r^{\prime}}(R)=T_{1}\left(L_{e}(R)\right)$.

(2) If $\left\{r, r^{\prime}\right\} \in$ edge $_{k} \mathcal{S}(\Gamma), 2 \leq k \leq n-1$, and $e=s_{k}(r)=s_{k}\left(r^{\prime}\right)$, then $\mathcal{A}_{r, r^{\prime}}(R)=T_{k}\left(C_{e}(R)\right)$.

(3) If $r=\left(i_{0}, \ldots, i_{n-2}, i_{n-1}\right)$ and $r^{\prime}=\left(i_{0}, \ldots, i_{n-2}, i_{n}\right)$ and $e^{*}=\left[i_{0}, \ldots, i_{n-2}\right.$, $\left.i_{n-1}, i_{n}\right] \in \Gamma_{*}^{n}$, i.e. $\left(r, r^{\prime}\right) \in$ edge $e_{n} \mathcal{S}\left(\Gamma^{*}\right)$, then $\mathcal{A}_{r, r^{\prime}}(R)=T_{n}\left(Z_{e^{*}}(R)\right)$.

Proof. (1) We begin by considering an edge such as $e=\left\{r=\left(i_{0}, i_{1}, i_{2}, \ldots, i_{n-1}\right)\right.$, $\left.r^{\prime}=\left(i_{1}, i_{0}, i_{2}, \ldots, i_{n-1}\right)\right\}$, where $r, r^{\prime} \in$ vert $\mathcal{S}(\Gamma)$. Define $s=\left\{i_{0}, i_{1}\right\}$, which determines an edge in the tree $\left(\Gamma^{0}, \Gamma^{1}\right)$ between the 0 -simplices $\left\{i_{0}\right\}$ and $\left\{i_{1}\right\}$. If $E_{r}(R)=\left(\mathbf{e}_{0}, \mathbf{e}_{1}, \ldots, \mathbf{e}_{n}\right)$, then

$$
E_{r}(R) T_{1}\left(L_{s}(R)\right)=\left(\mathbf{e}_{0}+L_{s}(R) \mathbf{e}_{1},-\mathbf{e}_{1}, \mathbf{e}_{2}, \ldots, \mathbf{e}_{n-1},-\mathbf{e}_{n}\right) .
$$

Our definitions immediately imply that this is the pose $E_{r^{\prime}}(R)$.

(2) Now suppose $\left\{r=\left(i_{0}, \ldots, i_{n-1}\right), r^{\prime}=\left(j_{0}, \ldots, j_{n-1}\right)\right\} \in$ edge $\mathcal{S}(\Gamma)$ where for some $2 \leq k \leq n-1$ we have

$$
\begin{aligned}
\left(i_{0}, \ldots, i_{k-2}\right) & =\left(j_{0}, \ldots, j_{k-2}\right), \\
\left(i_{k+1}, \ldots, i_{n-1}\right) & =\left(j_{k+1}, \ldots, j_{n-1}\right), \text { and } \\
\left(i_{k-1}, i_{k}\right) & =\left(j_{k}, j_{k-1}\right) .
\end{aligned}
$$


$s_{k}(r)=\left\{i_{0}, \ldots, i_{k}\right\}$ is equal to $s_{k}\left(r^{\prime}\right)=\left\{j_{0}, \ldots, j_{k}\right\}$, and corresponds to an edge $e$ in the tree $\left(\Gamma^{k-1}, \Gamma^{k}\right)$ with vertices $v_{1}=s_{k-1}(r)$ and $v_{2}=s_{k-1}\left(r^{\prime}\right)$ in $\Gamma^{k-1}$. Let $u=v_{1} \cap v_{2}=s_{k-2}(r)=s_{k-2}\left(r^{\prime}\right) \in \Gamma^{k-2}$. Let $E_{r}(R)=\left(\mathbf{e}_{0}, \mathbf{e}_{1}, \ldots, \mathbf{e}_{n}\right)$ and $E_{r^{\prime}}(R)=\left(\mathbf{e}_{0}^{\prime}, \mathbf{e}_{1}^{\prime}, \ldots, \mathbf{e}_{n}^{\prime}\right)$. Then for $C=C_{e}(R)$ note that

$$
E_{r}(R) T_{k}(C)=\left(\mathbf{e}_{0}, \ldots, \mathbf{e}_{k-2}, \mathbf{e}_{k-1} C+\mathbf{e}_{k} S, \mathbf{e}_{k-1} S-\mathbf{e}_{k} C, \mathbf{e}_{k+1}, \ldots, \mathbf{e}_{n-1},-\mathbf{e}_{n}\right) .
$$

Since $\left(i_{0}, \ldots, i_{k-2}\right)=\left(j_{0}, \ldots, j_{k-2}\right)$ it is clear that $\mathbf{e}_{0}^{\prime}=\mathbf{e}_{0}, \ldots, \mathbf{e}_{k-2}^{\prime}=\mathbf{e}_{k-2}$. We claim that $\mathbf{e}_{k-1}^{\prime}=\mathbf{e}_{k-1} C+\mathbf{e}_{k} S$. To see this, note that $1-\Pi_{v_{1}}=\left(1-\mathbf{e}_{k-1} \mathbf{e}_{k-1}^{T}\right)(1-$ $\left.\Pi_{u}\right)$. Therefore $\mathbf{e}_{k}$ is the unit vector in the direction of $\left(1-\Pi_{v_{1}}\right)\left(\mathbf{R}_{i_{k}}-\mathbf{R}_{i_{0}}\right)=$ $\left(1-\mathbf{e}_{k-1} \mathbf{e}_{k-1}^{T}\right)\left(1-\Pi_{u}\right)\left(\mathbf{R}_{i_{k}}-\mathbf{R}_{i_{0}}\right)$. Since $j_{k-1}=i_{k}$ it follows that $\mathbf{e}_{k-1}^{\prime}$ is the unit vector in the direction of $\left(1-\Pi_{u}\right)\left(\mathbf{R}_{i_{k}}-\mathbf{R}_{i_{0}}\right)$, and thus that $\mathbf{e}_{k}$ is the unit vector in the direction of $\left(1-\mathbf{e}_{k-1} \mathbf{e}_{k-1}^{T}\right) \mathbf{e}_{k-1}^{\prime}$. Thus there is a positive constant $\alpha$ such that $\mathbf{e}_{k} \alpha=\mathbf{e}_{k-1}^{\prime}-\mathbf{e}_{k-1}\left(\mathbf{e}_{k-1} \cdot \mathbf{e}_{k-1}^{\prime}\right)$. But $\mathbf{e}_{k-1} \cdot \mathbf{e}_{k-1}^{\prime}=C_{e}(R)=C$. Thus $\mathbf{e}_{k-1}^{\prime}=\mathbf{e}_{k-1} C+\mathbf{e}_{k} \alpha$. Since $\mathbf{e}_{k-1}^{\prime}$ is a unit vector and $\left\{\mathbf{e}_{k-1}, \mathbf{e}_{k}\right\}$ is an orthonormal set we have that $C^{2}+\alpha^{2}=1$. Since $\alpha>0$ we have $\alpha=\sqrt{1-C^{2}}=S$. Thus $\mathbf{e}_{k-1}^{\prime}=\mathbf{e}_{k-1} C+\mathbf{e}_{k} S$, as claimed. In the same vein we claim that $\mathbf{e}_{k}^{\prime}=\mathbf{e}_{k-1} S-\mathbf{e}_{k} C$. To see this, note that $1-\Pi_{v_{2}}=\left(1-\mathbf{e}_{k-1}^{\prime}\left(\mathbf{e}_{k-1}^{\prime}\right)^{T}\right)\left(1-\Pi_{u}\right)$. $\mathbf{e}_{k}^{\prime}$ is the unit vector in the direction of $\left(1-\Pi_{v_{2}}\right)\left(\mathbf{R}_{j_{k}}-\mathbf{R}_{j_{0}}\right)=\left(1-\mathbf{e}_{k-1}^{\prime}\left(\mathbf{e}_{k-1}^{\prime}\right)^{T}\right)\left(1-\Pi_{u}\right)\left(\mathbf{R}_{i_{k-1}}-\mathbf{R}_{i_{0}}\right)$, or of $\left(1-\mathbf{e}_{k-1}^{\prime}\left(\mathbf{e}_{k-1}^{\prime}\right)^{T}\right) \mathbf{e}_{k-1}$. Hence there is a positive constant $\beta$ such that $\mathbf{e}_{k}^{\prime} \beta=$ $\mathbf{e}_{k-1}-\mathbf{e}_{k-1}^{\prime}\left(\mathbf{e}_{k-1}^{\prime} \cdot \mathbf{e}_{k-1}\right)=\mathbf{e}_{k-1}-\mathbf{e}_{k-1}^{\prime} C_{e}(R)$. Using our known expression for $\mathbf{e}_{k-1}^{\prime}$, we can easily derive the claim from this. Since $\left(i_{k+1}, \ldots, i_{n-1}\right)=\left(j_{k+1}, \ldots, j_{n-1}\right)$ and $s_{j}(r)=s_{j}\left(r^{\prime}\right)$ for $j=k, \ldots, n-2$, we see that $\mathbf{e}_{k+1}^{\prime}=\mathbf{e}_{k+1}, \ldots, \mathbf{e}_{n-1}^{\prime}=\mathbf{e}_{n-1}$. Finally, $\mathbf{e}_{n}^{\prime}$ is either $\mathbf{e}_{n}$ or $-\mathbf{e}_{n}$, and it must be the latter since $\operatorname{det}\left(\begin{array}{cc}C & S \\ S & -C\end{array}\right)=-1$. Thus we have shown that $E_{r}(R) T_{k}\left(C_{e}(R)\right)=E_{r^{\prime}}(R)$.

(3) Now consider a pair $\left(r, r^{\prime}\right) \in$ edge $_{n} \mathcal{S}\left(\Gamma^{*}\right)$ of the form $r=\left(i_{0}, \ldots, i_{n-2}, i_{n-1}\right)$, $r^{\prime}=\left(i_{0}, \ldots, i_{n-2}, i_{n}\right)$, where $e^{*}=\left[i_{0}, \ldots, i_{n-1}, i_{n}\right] \in \Gamma_{*}^{n}, v=\left\{i_{0}, \ldots, i_{n-2}, i_{n-1}\right\} \in$ $\Gamma^{n-1}, v^{\prime}=\left\{i_{0}, \ldots, i_{n-2}, i_{n}\right\} \in \Gamma^{n-1}, e=v \cup v^{\prime} \in \Gamma^{n}$, and $s=v \cap v^{\prime} \in \Gamma^{n-2}$. Let $E_{r}(R)=\left(\mathbf{e}_{0}, \mathbf{e}_{1}, \ldots, \mathbf{e}_{n}\right)$ and $E_{r^{\prime}}(R)=\left(\mathbf{e}_{0}^{\prime}, \mathbf{e}_{1}^{\prime}, \ldots, \mathbf{e}_{n}^{\prime}\right)$. Clearly we have $\mathbf{e}_{k}^{\prime}=\mathbf{e}_{k}$ for $k=0,1, \ldots, n-2$. Then our definitions show that $C_{e}(R)=\mathbf{e}_{n-1} \cdot \mathbf{e}_{n-1}^{\prime}$ and $S_{e^{*}}(R)=\mathbf{e}_{n} \cdot \mathbf{e}_{n-1}^{\prime}$. Since $\mathbf{e}_{n-1}^{\prime}$ is in the two-dimensional space perpendicular to the span of $\left\{\mathbf{e}_{1}, \ldots, \mathbf{e}_{n-2}\right\}$, and $\left\{\mathbf{e}_{n-1}, \mathbf{e}_{n}\right\}$ is an orthonormal basis of this subspace, then we have that $\mathbf{e}_{n-1}^{\prime}=\mathbf{e}_{n-1} C_{e}(R)+\mathbf{e}_{n} S_{e^{*}}(R)$. Also the vector $-\mathbf{e}_{n-1} S_{e^{*}}(R)+$ $\mathbf{e}_{n} C_{e}(R)$ is a unit vector perpendicular to the span of $\left\{\mathbf{e}_{1}^{\prime}, \ldots, \mathbf{e}_{n-2}^{\prime}, \mathbf{e}_{n-1}^{\prime}\right\}$ and

$$
\begin{aligned}
\operatorname{det}\left(\mathbf{e}_{1}^{\prime}, \ldots,\right. & \left.\mathbf{e}_{n-2}^{\prime}, \mathbf{e}_{n-1}^{\prime},-\mathbf{e}_{n-1} S_{e^{*}}(R)+\mathbf{e}_{n} C_{e}(R)\right) \\
& =\operatorname{det}\left(\mathbf{e}_{1}, \ldots, \mathbf{e}_{n-2}, \mathbf{e}_{n-1} C_{e}(R)+\mathbf{e}_{n} S_{e^{*}}(R),-\mathbf{e}_{n-1} S_{e^{*}}(R)+\mathbf{e}_{n} C_{e}(R)\right) \\
& =\operatorname{det}\left(\mathbf{e}_{1}, \ldots, \mathbf{e}_{n}\right) \operatorname{det}\left(\begin{array}{cc}
C_{e}(R) & -S_{e^{*}}(R) \\
S_{e^{*}}(R) & C_{e}(R)
\end{array}\right)=1
\end{aligned}
$$

Hence $\mathbf{e}_{n}^{\prime}=-\mathbf{e}_{n-1} S_{e^{*}}(R)+\mathbf{e}_{n} C_{e}(R)$. We have shown that $E_{r}(R) T_{n}\left(C_{e}(R)+\right.$ $\left.i S_{e^{*}}(R)\right)=E_{r^{\prime}}(R)$.

Thus given $R \in \mathcal{B}(\Gamma)$ we can label each edge of $\mathcal{S}\left(\Gamma^{*}\right)$ with an element $\mathcal{A}_{r, r^{\prime}}(R) \in$ $G_{p}$ such that $E_{r}(R) \mathcal{A}_{r, r^{\prime}}(R)=E_{r^{\prime}}(R)$. If $(\mathbf{b}, A) \in G_{a}$ then

$$
\begin{aligned}
E_{r}((\mathbf{b}, A) \cdot R) \mathcal{A}_{r, r^{\prime}}(R) & =(\mathbf{b}, A) \cdot E_{r}(R) \mathcal{A}_{r, r^{\prime}}(R)=(\mathbf{b}, A) \cdot E_{r^{\prime}}(R)=E_{r^{\prime}}((\mathbf{b}, A) \cdot R) \\
& =E_{r}((\mathbf{b}, A) \cdot R) \mathcal{A}_{r, r^{\prime}}((\mathbf{b}, A) \cdot R),
\end{aligned}
$$


and hence $\mathcal{A}_{r, r^{\prime}}((\mathbf{b}, A) \cdot R)=\mathcal{A}_{r, r^{\prime}}(R)$. The labeling of the edges of the site graph $\mathcal{S}\left(\Gamma^{*}\right)$ with elements of the group $G_{p}$ resembles the cocycle construction in nonabelian sheaf cohomology [5]. Graphs whose edges are labeled with elements of a group have been called gain graphs [92].

Since the numbers $L, C$, and $Z$ can be uniquely recovered from the matrices $T_{1}(L), T_{k}(C)$, and $T_{n}(Z)$, and every element of $\Gamma^{k}, 1 \leq k \leq n$, can be associated with at least one element of edge $\mathcal{S}(\Gamma)$, this theorem gives another way to recover the coordinate functions $L_{e}(R), C_{e}(R)$, and $Z_{e^{*}}(R)$ from the configuration $R$. The relation $\mathcal{A}_{r, r^{\prime}}((\mathbf{b}, A) \cdot R)=\mathcal{A}_{r, r^{\prime}}(R)$ implies (although this is easy enough to check directly from the definitions) that

$$
\begin{aligned}
L_{e}((\mathbf{b}, A) \cdot R) & =L_{e}(R) & & e \in \Gamma^{1}, \\
C_{e}((\mathbf{b}, A) \cdot R) & =C_{e}(R) & & e \in \Gamma^{k}, 2 \leq k \leq n-1, \\
Z_{e^{*}}((\mathbf{b}, A) \cdot R) & =Z_{e^{*}}(R) & & e^{*} \in \Gamma_{*}^{n},
\end{aligned}
$$

for all $(\mathbf{b}, A) \in G_{a}$ and all $R \in \mathcal{B}(\Gamma)$. Since the left action of $G_{a}$ on $\mathcal{B}(\Gamma)$ is fixed point free and proper, $\mathcal{D}_{C}(\Gamma)=G_{a} \| \mathcal{B}(\Gamma)$ is a smooth manifold, and the projection mapping $\rho: \mathcal{B}(\Gamma) \rightarrow \mathcal{D}_{C}(\Gamma)$ is a smooth submersion (see Proposition 4.1.23 on page 266 of [2]). By "passage to quotients" the (well-defined) mapping $\hat{L}_{e}: \mathcal{D}_{C}(\Gamma) \rightarrow(0, \infty): G_{a} R \mapsto L_{e}(R)$ is smooth, for each $e \in \Gamma^{1}$. Likewise the mappings $\hat{C}_{e}: \mathcal{D}_{C}(\Gamma) \rightarrow(-1,1): G_{a} R \mapsto C_{e}(R)$ and $\hat{Z}_{e^{*}}: \mathcal{D}_{C}(\Gamma) \rightarrow S^{1}: G_{a} R \mapsto$ $Z_{e^{*}}(R)$ are smooth.

3.4. Labeling the Site Network. Part (3) of the above theorem tells us how to label a pair $\left(r, r^{\prime}\right) \in$ edge $_{n} \mathcal{S}\left(\Gamma^{*}\right)$ with an element $a_{r r^{\prime}}(R)=Z_{e^{*}}(R)$ of the group $S^{1}$, so that $E_{r}(R) T_{n}\left(a_{r r^{\prime}}(R)\right)=E_{r^{\prime}}(R)$. However, edge ${ }_{n} \mathcal{S}\left(\Gamma^{*}\right) \subset \mathcal{G}(\Gamma)$ and we will now show that the labeling can be uniquely extended to a mapping $a .(R): \mathcal{G}(\Gamma) \rightarrow$ $S^{1}:\left(r, r^{\prime}\right) \mapsto a_{r r^{\prime}}(R)$ such that $E_{r}(R) T_{n}\left(a_{r r^{\prime}}(R)\right)=E_{r^{\prime}}(R)$ holds for all $\left(r, r^{\prime}\right) \in$ $\mathcal{G}(\Gamma)$, or equivalently, $a_{r_{1} r_{3}}(R)=a_{r_{1} r_{2}}(R) a_{r_{2} r_{3}}(R)$ for all $\left(r_{1}, r_{2}\right),\left(r_{2}, r_{3}\right) \in \mathcal{G}(\Gamma)$. Mappings $a .(R)$ with this property are said to be $S^{1}$-valued cocycles, or groupoid homomorphisms. The mapping $a .(R): \mathcal{G}(\Gamma) \rightarrow S^{1}$ is useful when relating two Zsystems which differ only in the edges of the highest level tree; this situation can arise when Z-systems are tethered (see section 6).

To see how this extended labeling should be done we proceed as follows. We should label all pairs $(r, r)$ with the number 1, i.e. $a_{r r}(R)=1$. Furthermore, if $\left(r, r^{\prime}\right) \in$ edge $_{n} \mathcal{S}\left(\Gamma^{*}\right)$ then we should define $a_{r^{\prime} r}(R)=a_{r r^{\prime}}(R)^{-1}$. But these instructions might not label all the pairs in $\mathcal{G}(\Gamma)$, although they do suffice for the pair groupoid of ammonia (see Figure 6). So suppose $\left(r, r^{\prime}\right) \in \mathcal{G}(\Gamma)$ is one of the pairs not yet labeled. Let $r=\left(i_{0}, \ldots, i_{n-2}, i\right), r^{\prime}=\left(i_{0}, \ldots, i_{n-2}, i^{\prime}\right)$; since $r$ and $r^{\prime}$ are sites we have $v=\left\{i_{0}, \ldots, i_{n-2}, i\right\} \in \Gamma^{n-1}, v^{\prime}=\left\{i_{0}, \ldots, i_{n-2}, i^{\prime}\right\} \in \Gamma^{n-1}$, and $s=\left\{i_{0}, \ldots, i_{n-2}\right\} \in \Gamma^{n-2}$. We are assuming that $v \cup v^{\prime} \notin \Gamma^{n}$ and $i \neq i^{\prime}$, so that $s=v \cap v^{\prime}$. We need the following result when $k=n$.

Lemma. Suppose $\Gamma$ is an unoriented Z-system, $1 \leq k \leq n$, and $v$ and $v^{\prime}$ are distinct vertices in the tree $\left(\Gamma^{k-1}, \Gamma^{k}\right)$, where $v \cup v^{\prime} \notin \Gamma^{k}$ and $s=v \cap v^{\prime} \in \Gamma^{k-2}$. Let $\left(v=v_{0}, e_{1}, v_{1}, \ldots, v_{m-1}, e_{m}, v_{m}=v^{\prime}\right)$ be the unique path $(m \geq 2)$ connecting them. (The distinct vertices $v_{j} \in \Gamma^{k-1}$ and the distinct edges $e_{j} \in \Gamma^{k}$ are such that $e_{j}$ is incident on $v_{j-1}$ and $v_{j}$.) Then $v_{j-1} \cap v_{j}=s$ for all $j=1, \ldots, m$.

Proof. If $k=1$ then $\Gamma^{-1}=\{\emptyset\}$, and so the assertion of the lemma is trivial. So suppose $k \geq 2$. Consider the list $\left(v_{0} \cap v_{1}, v_{1} \cap v_{2}, \ldots, v_{m-1} \cap v_{m}, s\right)$ of simplices in 
$\Gamma^{k-2}$. By the remark after the lemma of section 2.7 we have that the unique path in the tree $\left(\Gamma^{k-2}, \Gamma^{k-1}\right)$ connecting $s$ to $s$ must pass through all the vertices in this list. Hence they must all be equal to $s$. Note that this implies that $J=0$ in the notation of that lemma.

Each vertex $v_{j}$ in the list $v_{0}, v_{1}, \ldots, v_{m}$ determines a site $r_{j}=\left(i_{0}, \ldots, i_{n-2}, i(j)\right)$ where $\{i(j)\}=v_{j} \backslash s$. Furthermore, for each $j=1, \ldots, m$, the label $a_{r_{j-1} r_{j}}(R)$ has been previously assigned, since $e_{j}=v_{j-1} \cup v_{j}=\left\{i_{0}, \ldots, i_{n-2}, i(j-1), i(j)\right\} \in \Gamma^{n}$. Therefore we define

$$
a_{r r^{\prime}}(R)=a_{r_{0} r_{1}}(R) a_{r_{1} r_{2}}(R) \ldots a_{r_{m-1} r_{m}}(R) .
$$

This is a complete and unique way to define the labels so that the result is a groupoid homomorphism.

Thus a labeled Z-system induces a labeling of the edges of the site network with appropriate elements of $G_{p}$. This pattern of labeling is consistent with a labeling of the sites with poses arising from a choice of configuration $R \in \mathcal{B}(\Gamma)$. A labeling of the edges of the site network is called balanced if the product in $G_{p}$ of the labels of the edges of any cycle in the site network is the identity. Every labeling arising from a configuration is balanced. It would be interesting to give an effective algebraic characterization of the class of labelings of the site network arising from configurations. Perhaps this would give a theoretically attractive alternative way to understand internal coordinates avoiding a choice of $\Gamma^{n}$ or $\Gamma_{*}^{n}$.

The labeled site graph and the labeled site network provide a convenient formalism for the detailed study of molecular shapes [33], [40], [78]; c.f. sections 6.5, 6.6, and 6.7. Both structures are compactly coded by and most easily manipulated in terms of the underlying Z-system.

3.5. Polyspherical Coordinate Mapping. If a distinguished site $r$ is chosen for an unoriented Z-system $\Gamma$ it will be called a root of $\Gamma$, because $s_{k}(r)$ is thought of as a choice of root vertex for the tree $\left(\Gamma^{k}, \Gamma^{k+1}\right)$ for all $k=0, \ldots, n-1$.

Definition. Suppose $\Gamma^{*}$ is a Z-system and $r$ is a root for $\Gamma$. Then define the mapping $\eta: \mathcal{B}(\Gamma) \rightarrow \mathcal{P} \times \mathcal{D}_{P}\left(\Gamma^{*}\right)$ by the rule

$$
R \mapsto\left(E_{r}(R), e \in \Gamma^{1} \mapsto L_{e}(R),\left\{e \in \Gamma^{k} \mapsto C_{e}(R)\right\}_{k=2}^{n-1}, e^{*} \in \Gamma_{*}^{n} \mapsto Z_{e^{*}}(R)\right) .
$$

We will call this the polyspherical trivialization associated to $\left(\Gamma^{*}, r\right)$. Define also the mapping $\hat{\eta}: \mathcal{D}_{C}(\Gamma) \rightarrow \mathcal{D}_{P}\left(\Gamma^{*}\right)$ by the rule:

$$
\mathcal{O} \mapsto\left(e \in \Gamma^{1} \mapsto \hat{L}_{e}(\mathcal{O}),\left\{e \in \Gamma^{k} \mapsto \hat{C}_{e}(\mathcal{O})\right\}_{k=2}^{n-1}, e^{*} \in \Gamma_{*}^{n} \mapsto \hat{Z}_{e^{*}}(\mathcal{O})\right) .
$$

We will call this the polyspherical coordinate mapping associated to $\Gamma^{*}$. Thus $\eta=$ $\left(E_{r}, \hat{\eta} \circ \rho\right)$.

$\eta$ and $\hat{\eta}$ are clearly smooth mappings. In fact $\eta$ is algebraic.

It may not yet be clear why we use the term "polyspherical" in conjunction with these mappings. This will become clear in the next section where we learn how to invert the mapping $\eta$.

\section{The Main Theorems}

4.1. The Coordinatization Theorem. A comparison of the dimensions of $\mathcal{B}(\Gamma)$ and $\mathcal{P} \times \mathcal{D}_{P}\left(\Gamma^{*}\right)$ suggests that the mapping $\eta$ defined in the previous section might be invertible. $\mathcal{B}(\Gamma)$ is a dense open subset of $\left(\mathbb{R}^{n}\right)^{\mathcal{N}}$ and so has dimension $n N$. $\mathcal{P}$ 
is diffeomorphic to $\mathbb{R}^{n} \times \mathrm{SO}(n)$, and so has dimension $n+n(n-1) / 2=n(n+1) / 2$. $(\mathrm{SO}(n)$ has the same dimension as its Lie algebra, namely the set of all $n \times n$ real antisymmetric matrices.) Since $\left|\Gamma^{0}\right|=N$ and $\left(\Gamma^{0}, \Gamma^{1}\right)$ is a tree we have that $\left|\Gamma^{1}\right|=N-1$. Similarly $\left|\Gamma^{k}\right|=N-k$, for $k=1, \ldots, n$. Thus $\mathcal{D}_{P}\left(\Gamma^{*}\right)$ has dimension $\sum_{k=1}^{n}(N-k)=n N-n(n+1) / 2$. Therefore $\mathcal{P} \times \mathcal{D}_{P}\left(\Gamma^{*}\right)$ also has dimension $n N$, as claimed.

Theorem. Suppose $N \geq n \geq 2, \Gamma^{*}$ is an $n$-dimensional Z-system on the set $\mathcal{N}, r$ is a root for $\Gamma$, and $\eta: \mathcal{B}(\Gamma) \rightarrow \mathcal{P} \times \mathcal{D}_{P}\left(\Gamma^{*}\right)$ is the polyspherical trivialization defined in the previous section. Then $\eta$ is a diffeomorphism. Furthermore the polyspherical coordinate mapping $\hat{\eta}: \mathcal{D}_{C}(\Gamma) \rightarrow \mathcal{D}_{P}\left(\Gamma^{*}\right)$ associated to $\Gamma^{*}$ is also a diffeomorphism.

Proof. We will proceed by constructing the inverse $\zeta: \mathcal{P} \times \mathcal{D}_{P}\left(\Gamma^{*}\right) \rightarrow \mathcal{B}(\Gamma)$ to $\eta$ via induction on $N$. We start with the case $N=n$. So let $(E, \gamma) \in \mathcal{P} \times \mathcal{D}_{P}\left(\Gamma^{*}\right)$ be given, where $E=\left(\mathbf{e}_{0}, \mathbf{e}_{1}, \ldots, \mathbf{e}_{n}\right) \in \mathcal{P}$ and

$$
\gamma=\left(e \in \Gamma^{1} \mapsto L_{e},\left\{e \in \Gamma^{k} \mapsto C_{e}\right\}_{k=2}^{n-1}\right) \in \mathcal{D}_{P}\left(\Gamma^{*}\right) .
$$

When $N=n$ we have $\Gamma^{n}=\emptyset$, so we have not included any parameters of type $Z_{e^{*}}$ in the definition of $\gamma$. Let $r=\left(i_{0}, i_{1}, \ldots, i_{n-1}\right)$ be the root site. Define $\mathbf{R}_{i_{0}}=\mathbf{e}_{0}$, and $\mathbf{R}_{i_{1}}=\mathbf{R}_{i_{0}}+\mathbf{e}_{1} L_{\left\{i_{0}, i_{1}\right\}}$. Because $\gamma \in \mathcal{D}_{P}\left(\Gamma^{*}\right)$ we have $L_{\left\{i_{0}, i_{1}\right\}}>0$, and hence the 1simplex $\left\{\mathbf{R}_{i_{0}}, \mathbf{R}_{i_{1}}\right\}$ is geometrically independent and $\operatorname{span}\left\{\mathbf{R}_{i_{1}}-\mathbf{R}_{i_{0}}\right\}=\operatorname{span}\left\{\mathbf{e}_{1}\right\}$. Now suppose $\mathbf{R}_{i_{j}}$ has been defined for all $0 \leq j \leq k-1$, where $2 \leq k \leq n-1$. Assume that the $(k-1)$-simplex $\left\{\mathbf{R}_{i_{0}}, \ldots, \mathbf{R}_{i_{k-1}}\right\}$ is geometrically independent and span $\left\{\mathbf{R}_{i_{1}}-\mathbf{R}_{i_{0}}, \ldots, \mathbf{R}_{i_{k-1}}-\mathbf{R}_{i_{0}}\right\}=$ span $\left\{\mathbf{e}_{1}, \ldots, \mathbf{e}_{k-1}\right\}$. We wish to define $\mathbf{R}_{i_{k}}$ so that these properties are extended.

$s_{k}^{1}=s_{k}(r)=\left\{i_{0}, i_{1}, \ldots, i_{k}\right\} \in \Gamma^{k}$ is incident on two $(k-1)$-simplices in $\Gamma^{k-1}$, one of which is $s_{k-1}^{0}=s_{k-1}(r)=\left\{i_{0}, \ldots, i_{k-1}\right\}$ and the other we will denote by $s_{k-1}^{1}$; the intersection of these two being denoted by $s_{k-2}^{0} \in \Gamma^{k-2}$. Define $j_{k-1}$ by the relation $s_{k-1}^{0} \backslash s_{k-2}^{0}=\left\{j_{k-1}\right\}$. Notice that $s_{k-1}^{1} \backslash s_{k-2}^{0}=\left\{i_{k}\right\}$.

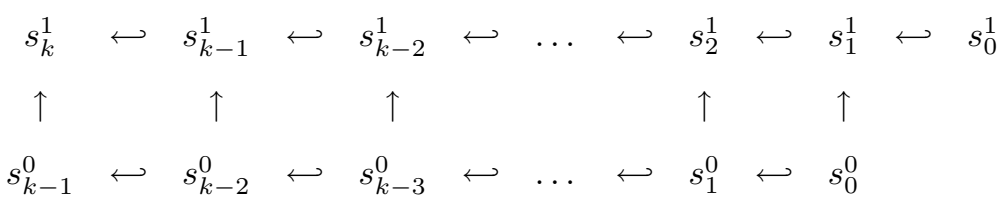

If the smallest simplex in this array is a 0-simplex then we stop, otherwise we continue as follows: $s_{k-1}^{1}$ is incident on $s_{k-2}^{0}$ and one other $(k-2)$-simplex, call it $s_{k-2}^{1} \in \Gamma^{k-2}$. The intersection of $s_{k-2}^{0}$ and $s_{k-2}^{1}$ is a $(k-3)$-simplex called $s_{k-3}^{0} \in$ $\Gamma^{k-3}$. Define $j_{k-2}$ such that $s_{k-2}^{0} \backslash s_{k-3}^{0}=\left\{j_{k-2}\right\}$. Notice that $s_{k-2}^{1} \backslash s_{k-3}^{0}=\left\{i_{k}\right\}$. When this process stops we will have that $s_{0}^{0}$ and $s_{0}^{1}=\left\{i_{k}\right\}$ are 0 -simplices. Let $s_{0}^{0}=\left\{j_{0}\right\}$. Then $s_{h}^{0}=\left\{j_{0}, \ldots, j_{h}\right\}$ and $s_{h}^{1}=\left\{j_{0}, \ldots, j_{h-1}, i_{k}\right\}$, for $h=0, \ldots, k$. Thus $\left\{j_{0}, \ldots, j_{k-1}\right\}=s_{k-1}^{0}=\left\{i_{0}, \ldots, i_{k-1}\right\}$, and hence $\mathbf{R}_{j_{0}}, \ldots, \mathbf{R}_{j_{k-1}}$ are already defined. Thus the following are sites of our Z-system:

$$
\begin{aligned}
r_{k} & =\left(j_{0}, \ldots, j_{k-2}, j_{k-1}, i_{k}, i_{k+1}, \ldots, i_{n-1}\right) \\
\times & T_{k}\left(C_{s_{k}^{1}}\right) \\
r_{k-1} & =\left(j_{0}, \ldots, j_{k-2}, i_{k}, j_{k-1}, i_{k+1}, \ldots, i_{n-1}\right) \\
& \vdots \\
r_{2} & =\left(j_{0}, j_{1}, i_{k}, j_{2}, \ldots, j_{k-1}, i_{k+1}, \ldots, i_{n-1}\right)
\end{aligned}
$$




$$
\begin{array}{cc}
\quad \times & T_{2}\left(C_{s_{2}^{1}}\right) \\
r_{1}=\left(j_{0}, i_{k}, j_{1}, \ldots, j_{k-1}, i_{k+1}, \ldots, i_{n-1}\right) & \\
\times & T_{1}\left(L_{s_{1}^{1}}\right) \\
r_{0}=\left(i_{k}, j_{0}, j_{1}, \ldots, j_{k-1}, i_{k+1}, \ldots, i_{n-1}\right) &
\end{array}
$$

Each one is obtained from its predecessor by a single interchange of adjacent entries. Thus $\left\{r_{h}, r_{h-1}\right\}, h=1, \ldots, k$ is an edge of the undirected site graph. Define $E_{r_{k}}=\left(\mathbf{e}_{0}^{\prime}, \mathbf{e}_{1}^{\prime}, \ldots, \mathbf{e}_{k-1}^{\prime}, \mathbf{e}_{k}, \ldots, \mathbf{e}_{n-1}, \pm \mathbf{e}_{n}\right)$, where

$$
\mathbf{e}_{0}^{\prime}=\mathbf{R}_{j_{0}}, \quad \mathbf{e}_{h}^{\prime}=\frac{\left(1-\Pi_{s_{h-1}\left(r_{k}\right)}\right)\left(\mathbf{R}_{j_{h}}-\mathbf{R}_{j_{0}}\right)}{\left\|\left(1-\Pi_{s_{h-1}\left(r_{k}\right)}\right)\left(\mathbf{R}_{j_{h}}-\mathbf{R}_{j_{0}}\right)\right\|}, \quad h=1, \ldots, k-1,
$$

and where the sign is chosen to assure that this is indeed a pose. The orthogonal projection operators $\Pi_{s}$ are defined in section 3.1. Note that

$$
\begin{aligned}
\operatorname{span}\left\{\mathbf{e}_{1}^{\prime}, \ldots, \mathbf{e}_{k-1}^{\prime}\right\} & =\operatorname{span}\left\{\mathbf{R}_{j_{1}}-\mathbf{R}_{j_{0}}, \ldots, \mathbf{R}_{j_{k-1}}-\mathbf{R}_{j_{0}}\right\} \\
& =\operatorname{span}\left\{\mathbf{R}_{i_{1}}-\mathbf{R}_{i_{0}}, \ldots, \mathbf{R}_{i_{k-1}}-\mathbf{R}_{i_{0}}\right\} \\
& =\operatorname{span}\left\{\mathbf{e}_{1}, \ldots, \mathbf{e}_{k-1}\right\}
\end{aligned}
$$

and hence $\mathbf{e}_{k}, \ldots, \mathbf{e}_{n}$ are perpendicular to this span. Consider the product

$$
E_{r_{0}}=E_{r_{k}} \mathcal{A}_{r_{k} r_{k-1}} \ldots \mathcal{A}_{r_{2} r_{1}} \mathcal{A}_{r_{1} r_{0}}
$$

where $\mathcal{A}_{r_{1} r_{0}}=T_{1}\left(L_{s_{1}^{1}}\right)$ and $\mathcal{A}_{r_{h} r_{h-1}}=T_{h}\left(C_{s_{h}^{1}}\right), h=2, \ldots, k$. This should be a pose whose origin is located at $\mathbf{R}_{i_{k}}$, the quantity we are trying to define. Thus define $\mathbf{R}_{i_{k}}=E_{r_{0}}(1,0, \ldots, 0)^{T}$ or

$$
\begin{aligned}
\mathbf{R}_{i_{k}}=E_{r_{k}} T_{k}\left(C_{s_{k}^{1}}\right) T_{k-1}\left(C_{s_{k-1}^{1}}\right) \ldots T_{2}\left(C_{s_{2}^{1}}\right) T_{1}\left(L_{s_{1}^{1}}\right)\left(\begin{array}{c}
1 \\
0 \\
\vdots \\
0
\end{array}\right) \\
=\left(\mathbf{e}_{0}^{\prime}, \mathbf{e}_{1}^{\prime}, \ldots, \mathbf{e}_{k-1}^{\prime}, \mathbf{e}_{k}, \ldots, \mathbf{e}_{n-1}, \pm \mathbf{e}_{n}\right)\left(\begin{array}{c}
1 \\
C_{s_{2}^{1}} L_{s_{1}^{1}} \\
C_{s_{3}^{1}} S_{s_{2}^{1}} L_{s_{1}^{1}} \\
\vdots \\
C_{s_{k}^{1}} S_{s_{k-1}^{1}} \ldots S_{s_{2}^{1}} L_{s_{1}^{1}} \\
S_{s_{k}^{1}} S_{s_{k-1}^{1}}^{1} \ldots S_{s_{2}^{1}} L_{s_{1}^{1}} \\
0 \\
\vdots \\
0
\end{array}\right)
\end{aligned}
$$

In the above, we are using the notation $S_{s}=\sqrt{1-C_{s}^{2}}$. Because $\gamma \in \mathcal{D}_{P}\left(\Gamma^{*}\right)$ it follows that the component of $\mathbf{e}_{k}$ is nonzero, which implies that the simplex $\left\{\mathbf{R}_{i_{0}}, \ldots, \mathbf{R}_{i_{k}}\right\}$ is geometrically independent. By definition

$$
\mathbf{R}_{i_{k}}-\mathbf{R}_{j_{0}} \in \operatorname{span}\left\{\mathbf{e}_{1}^{\prime}, \ldots, \mathbf{e}_{k-1}^{\prime}, \mathbf{e}_{k}\right\}=\operatorname{span}\left\{\mathbf{e}_{1}, \ldots, \mathbf{e}_{k-1}, \mathbf{e}_{k}\right\} .
$$

Since $\mathbf{R}_{j_{0}}-\mathbf{R}_{i_{0}} \in \operatorname{span}\left\{\mathbf{e}_{1}^{\prime}, \ldots, \mathbf{e}_{k-1}^{\prime}\right\}=\operatorname{span}\left\{\mathbf{e}_{1}, \ldots, \mathbf{e}_{k-1}\right\}$, we have that $\mathbf{R}_{i_{k}}-$ $\mathbf{R}_{i_{0}}=\mathbf{R}_{i_{k}}-\mathbf{R}_{j_{0}}+\left(\mathbf{R}_{j_{0}}-\mathbf{R}_{i_{0}}\right) \in \operatorname{span}\left\{\mathbf{e}_{1}, \ldots, \mathbf{e}_{k-1}, \mathbf{e}_{k}\right\}$. Thus span $\left\{\mathbf{R}_{i_{1}}-\right.$ $\left.\mathbf{R}_{i_{0}}, \ldots, \mathbf{R}_{i_{k}}-\mathbf{R}_{i_{0}}\right\} \subset$ span $\left\{\mathbf{e}_{1}, \ldots, \mathbf{e}_{k}\right\}$. Since these subspaces have the same 
dimension, they are in fact equal. Clearly $\mathbf{R}_{i_{k}}$ does not depend on the vectors $\mathbf{e}_{k+1}, \ldots, \mathbf{e}_{n}$ at all.

Thus by induction all $n$ points $\left(\mathbf{R}_{i_{0}}, \ldots, \mathbf{R}_{i_{n-1}}\right)$ have now been defined and the resulting $(n-1)$-simplex is geometrically independent. This list of $n$ points defines the value of the mapping $\zeta$ on the argument $(E, \gamma)$. Clearly $\zeta$ is a smooth function. By the theorem of section 3.3 we have that $\eta \circ \zeta$ and $\zeta \circ \eta$ are identity mappings.

Now suppose for $N \geq n+1$ that a smooth inverse $\zeta$ exists for the polyspherical coordinate mapping $\eta$ for all $n$-dimensional Z-systems on any set of $N-1$ elements. Suppose $\mathcal{N}=\{1, \ldots, N\}$ and $\Gamma^{*}$ is an $n$-dimensional Z-system on $\mathcal{N}$, with its trivialization domain $\mathcal{B}(\Gamma)$, parameter domain $\mathcal{D}_{P}\left(\Gamma^{*}\right)$, root site $r=\left(i_{0}, \ldots, i_{n-1}\right)$, and smooth polyspherical trivialization mapping $\eta: \mathcal{B}(\Gamma) \rightarrow \mathcal{P} \times \mathcal{D}_{P}\left(\Gamma^{*}\right)$. The tree $\left(\Gamma^{n-1}, \Gamma^{n}\right)$ has leaves, which are vertices with degree one (having only one edge incident on them). Since $N \geq n+1$ we have $\left|\Gamma^{n-1}\right|=N-(n-1) \geq 2$. Thus, since every tree with at least two vertices has at least two leaves, we can choose a leaf vertex $s_{n-1}^{1} \in \Gamma^{n-1}$ which is not the root vertex $s_{n-1}(r)=\left\{i_{0}, \ldots, i_{n-1}\right\} \in \Gamma^{n-1}$. Let $s_{n}^{1} \in \Gamma^{n}$ denote the single edge incident on this leaf, and let $s_{n-1}^{0} \in \Gamma^{n-1}$ denote the other vertex on which this edge is incident. Define $s_{n-2}^{0}=s_{n-1}^{0} \cap s_{n-1}^{1} \in \Gamma^{n-2}$. Note that $s_{n-1}^{1}$ is an edge in the tree $\left(\Gamma^{n-2}, \Gamma^{n-1}\right)$ incident on the vertex $s_{n-2}^{0}$; hence there is exactly one other vertex $s_{n-2}^{1} \in \Gamma^{n-2}$ on which it is also incident. If the smallest of these simplices is a 0 -simplex then we stop the process, but otherwise we continue the process by defining $s_{n-3}^{0}=s_{n-2}^{0} \cap s_{n-2}^{1} \in \Gamma^{n-3}$ and noting that $s_{n-2}^{1}$ is an edge in the tree $\left(\Gamma^{n-3}, \Gamma^{n-2}\right)$ incident on the vertex $s_{n-3}^{0}$, and therefore also on exactly on other vertex $s_{n-3}^{1} \in \Gamma^{n-3}$. Continuing this process until the smallest simplices are 0 -simplices we obtain the following scheme:

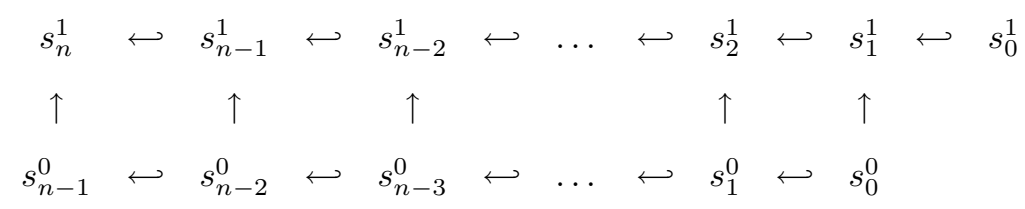

Suppose $s_{k-1}^{1}$ is a leaf vertex in the tree $\left(\Gamma^{k-1}, \Gamma^{k}\right)$ for some $2 \leq k \leq n$. We claim that $s_{k-2}^{1}$ is a leaf vertex in the tree $\left(\Gamma^{k-2}, \Gamma^{k-1}\right)$. Suppose by way of contradiction that $s_{k-2}^{1}$ is a vertex of degree at least two in the tree $\left(\Gamma^{k-2}, \Gamma^{k-1}\right)$, i.e. there is an edge $s \in \Gamma^{k-1}$ which is incident on $s_{k-2}^{1}$ and is distinct from $s_{k-1}^{1}$. Suppose $s=s_{k-1}^{0}$. Then $s_{k-2}^{0}=s_{k-1}^{0} \cap s_{k-1}^{1}=s \cap s_{k-1}^{1}=s_{k-2}^{1}$, which is a contradiction. So $s \neq s_{k-1}^{0}$. If $s \cup s_{k-1}^{1} \in \Gamma^{k}$ then $s_{k-1}^{1}$ would have two distinct edges incident on it, namely $s_{k}^{1}$ and $s \cup s_{k-1}^{1}$, contradicting the assumption that $s_{k-1}^{1}$ is a leaf. Therefore $s \cup s_{k-1}^{1} \notin \Gamma^{k}$. Consider the unique path in the tree $\left(\Gamma^{k-1}, \Gamma^{k}\right)$ connecting $s$ to $s_{k-1}^{1}$. This path must have length at least two, and must contain the edge $s_{k}^{1}$. By the lemma from section 3.4 the intersection of the pair of vertices for each edge of this path must be the same element of $\Gamma^{k-2}$. This element can be computed as the intersection of the two vertices of the edge $s_{k}^{1}$, namely $s_{k-1}^{0} \cap s_{k-1}^{1}=s_{k-2}^{0}$. But this would require that $s_{k-2}^{0} \subset s$, i.e. $s$ is incident on $s_{k-2}^{0}$, which is impossible, since $s$ would coincide with $s_{k-1}^{1}$, both being incident on $s_{k-2}^{0}$ and $s_{k-2}^{1}$. This contradiction shows that $s_{k-2}^{1}$ has degree one as a vertex in $\left(\Gamma^{k-2}, \Gamma^{k-1}\right)$, as claimed.

Thus each of $s_{n-1}^{1}, \ldots, s_{1}^{1}, s_{0}^{1}$ are leaf vertices in their respective trees. Define $\tilde{\Gamma}^{j}=\Gamma^{j} \backslash\left\{s_{j}^{1}\right\}$ for all $j=0,1, \ldots, n$. In each case we have deleted a leaf vertex and the single edge which was incident on it, so $\left(\tilde{\Gamma}^{j-1}, \tilde{\Gamma}^{j}\right)$ is a tree for all $j=1, \ldots, n$. 
If $v_{1}, v_{2} \in \tilde{\Gamma}^{j-1}$ satisfy $v_{1} \cup v_{2} \in \tilde{\Gamma}^{j}$ then $v_{1} \cap v_{2} \in \Gamma^{j-2}$. Since $v_{1} \neq s_{j-1}^{1}$ and $v_{2} \neq s_{j-1}^{1}$ and $s_{j-1}^{1}$ is the only simplex in $\Gamma^{j-1}$ which is incident on $s_{j-2}^{1}$, we have that $v_{1} \cap v_{2} \neq s_{j-2}^{1}$ and hence $v_{1} \cap v_{2} \in \tilde{\Gamma}^{j-2}$. Thus $\tilde{\Gamma}^{*}=\{\emptyset\} \cup \tilde{\Gamma}^{0} \cup \tilde{\Gamma}^{1} \cup \cdots \cup \tilde{\Gamma}_{*}^{n}$ is an $n$-dimensional Z-system on the set $\tilde{\mathcal{N}}=\mathcal{N} \backslash s_{0}^{1}$. If $s_{0}^{1} \subset s_{n-1}(r)$ then one, say $\tilde{s}_{n-2}$, of the two $(n-2)$-simplices in $\Gamma^{n-2}$ on which $s_{n-1}(r)$ is incident must satisfy $s_{0}^{1} \subset \tilde{s}_{n-2}$. Continuing this sort of argument, we show that $s_{0}^{1} \subset \tilde{s}_{1} \subset$ $\cdots \subset \tilde{s}_{n-2} \subset s_{n-1}(r)$. Since the only 1 -simplex in $\Gamma^{1}$ incident on $s_{0}^{1}$ is $s_{1}^{1}$ we must have $\tilde{s}_{1}=s_{1}^{1}$. Since the only 2 -simplex in $\Gamma^{2}$ incident on $s_{1}^{1}$ is $s_{2}^{1}$ we must have $\tilde{s}_{2}=s_{2}^{1}$. Continuing this argument we find that $s_{n-1}(r)=s_{n-1}^{1}$. This contradicts our original choice of the leaf $s_{n-1}^{1} \neq s_{n-1}(r)$. Therefore $s_{0}^{1} \not \subset s_{n-1}(r)$. Thus for all $0 \leq j \leq n-1$ we have $s_{j}(r) \neq s_{j}^{1}$, since $s_{0}^{1} \subset s_{j}^{1}$ but $s_{0}^{1} \not \subset s_{j}(r)$. Therefore $r$ is a root for the Z-system $\tilde{\Gamma}^{*}$.

Define $\mathcal{B}(\tilde{\Gamma})=\left\{\tilde{R}: \tilde{\mathcal{N}} \rightarrow \mathbb{R}^{n} \mid\right.$ for all $s \in \tilde{\Gamma}^{n-1}$ the simplex $R_{s}$ is geometrically independent $\}$. The restriction mapping maps $\left(\mathbb{R}^{n}\right)^{\mathcal{N}} \rightarrow\left(\mathbb{R}^{n}\right)^{\tilde{\mathcal{N}}}: R \mapsto \tilde{R}$. If $R \in \mathcal{B}(\Gamma)$ then clearly its restriction $\tilde{R}$ is in $\mathcal{B}(\tilde{\Gamma})$. Define $\mathcal{D}_{P}\left(\tilde{\Gamma}^{*}\right)=(0, \infty)^{\tilde{\Gamma}^{1}} \times$ $\left[\prod_{k=2}^{n-1}(-1,1)^{\tilde{\Gamma}^{k}}\right] \times\left(S^{1}\right)^{\tilde{\Gamma}_{*}^{n}}$. Clearly the restriction mapping $\gamma \mapsto \tilde{\gamma}$ maps $\mathcal{D}_{P}\left(\Gamma^{*}\right)$ onto $\mathcal{D}_{P}\left(\tilde{\Gamma}^{*}\right)$. If $\tilde{\eta}: \mathcal{B}(\tilde{\Gamma}) \rightarrow \mathcal{P} \times \mathcal{D}_{P}\left(\tilde{\Gamma}^{*}\right)$ is the polyspherical trivialization mapping for the rooted Z-system $\left(\tilde{\Gamma}^{*}, r\right)$ then we claim the following diagram commutes:

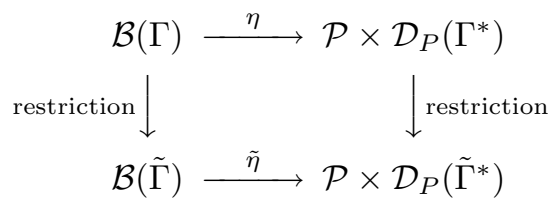

This is because the only simplices which contain $s_{0}^{1}=\{i\}$ as a subset are the ones which are excluded from $\tilde{\Gamma}^{j}$. Hence the labels of all the simplices in $\tilde{\Gamma}^{j}$ can be computed without knowing $\mathbf{R}_{i}$, and these computations are performed in exactly the same manner, whether by $\eta$ or by $\tilde{\eta}$. By the induction hypothesis there is a smooth inverse $\tilde{\zeta}: \mathcal{P} \times \mathcal{D}_{P}\left(\tilde{\Gamma}^{*}\right) \rightarrow \mathcal{B}(\tilde{\Gamma})$ to the mapping $\tilde{\eta}$.

Let $(E, \gamma) \in \mathcal{P} \times \mathcal{D}_{P}\left(\Gamma^{*}\right)$ be given, where

$$
\gamma=\left(e \in \Gamma^{1} \mapsto L_{e},\left\{e \in \Gamma^{k} \mapsto C_{e}\right\}_{k=2}^{n-1}, e^{*} \in \Gamma_{*}^{n} \mapsto Z_{e^{*}}\right) \in \mathcal{D}_{P}\left(\Gamma^{*}\right) .
$$

We wish to define $\zeta(E, \gamma) \in \mathcal{B}(\Gamma)$ such that the mapping $\zeta$ is a smooth inverse to the polyspherical coordinate mapping $\eta$. Define the site $r_{n}=\left(j_{0}, j_{1}, \ldots, j_{n-1}\right)$ such that $\left\{j_{0}, \ldots, j_{k}\right\}=s_{k}^{0} \in \tilde{\Gamma}^{k}$ for all $k=0,1, \ldots, n-1 . r_{n}$ is a site of the Z-system $\tilde{\Gamma}^{*}$. If $\tilde{R}=\tilde{\zeta}(E, \tilde{\gamma})$, then the pose $E_{r_{n}}(\tilde{R})=\left(\tilde{\mathbf{e}}_{0}, \tilde{\mathbf{e}}_{1}, \ldots, \tilde{\mathbf{e}}_{n}\right)$ is well-defined, and a smooth function of $(E, \gamma)$. To define $R=\zeta(E, \gamma)$ we augment $\tilde{R}$ by defining $\mathbf{R}_{i}$. Note that $s_{k}^{1}=\left\{j_{0}, \ldots, j_{k-1}, i\right\}$ for all $k=0,1, \ldots, n$. Thus we have the sequence of sites:

$$
\begin{array}{rlr}
r_{n} & =\left(j_{0}, j_{1}, j_{2}, j_{3}, \ldots, j_{n-2}, j_{n-1}\right) & \\
\times & T_{n}\left(\left(Z_{\left(s_{n}^{1}\right)^{*}}\right)^{a}\right) \\
r_{n-1} & =\left(j_{0}, j_{1}, j_{2}, j_{3}, \ldots, j_{n-2}, i\right) & \\
\vdots & & \\
r_{2} & =\left(j_{0}, j_{1}, i, j_{2}, \ldots, j_{n-3}, j_{n-2}\right) & \\
& \times & T_{2}\left(C_{s_{2}^{1}}\right)
\end{array}
$$




$$
\begin{aligned}
& r_{1}=\underset{\times}{\left(j_{0}, i, j_{1}, j_{2}, \ldots, j_{n-3}, j_{n-2}\right)} \\
& \quad \times \\
& r_{0}=\left(i, j_{0}, j_{1}, j_{2}, \ldots, j_{n-3}, j_{n-2}\right)
\end{aligned}
$$

$$
T_{1}\left(L_{s_{1}^{1}}\right)
$$

Define $E_{r_{0}}=E_{r_{n}}(\tilde{R}) \mathcal{A}_{r_{n} r_{n-1}} \ldots \mathcal{A}_{r_{2} r_{1}} \mathcal{A}_{r_{1} r_{0}}$, where $\mathcal{A}_{r_{1} r_{0}}=T_{1}\left(L_{s_{1}^{1}}\right), \mathcal{A}_{r_{k} r_{k-1}}=$ $T_{k}\left(C_{s_{k}^{1}}\right), k=2, \ldots, n-1$, and $\mathcal{A}_{r_{n} r_{n-1}}=T_{n}\left(\left(Z_{\left(s_{n}^{1}\right)^{*}}\right)^{a}\right)$, where

$$
a= \begin{cases}1 & \text { if }\left[j_{0}, \ldots, j_{n-2}, j_{n-1}, i\right]=\left(s_{n}^{1}\right)^{*} \\ -1 & \text { if }\left[j_{0}, \ldots, j_{n-2}, i, j_{n-1}\right]=\left(s_{n}^{1}\right)^{*}\end{cases}
$$

Thus $a=1$ if $\left(r_{n}, r_{n-1}\right) \in$ edge $_{n} \mathcal{S}\left(\Gamma^{*}\right)$ and $a=-1$ otherwise. We define $\mathbf{R}_{i}=$ $E_{r_{0}}(1,0, \ldots, 0)^{T}$. Thus

$$
\mathbf{R}_{i}=\left(\tilde{\mathbf{e}}_{0}, \tilde{\mathbf{e}}_{1}, \ldots, \tilde{\mathbf{e}}_{n}\right)\left(\begin{array}{c}
1 \\
C_{s_{2}^{1}} L_{s_{1}^{1}} \\
C_{s_{3}^{1}} S_{s_{2}^{1}} L_{s_{1}^{1}} \\
\vdots \\
C_{s_{n}^{1}} S_{s_{n-1}^{1}} \ldots S_{s_{2}^{1}} L_{s_{1}^{1}} \\
a S_{\left(s_{n}^{1}\right) *} S_{s_{n-1}^{1}} \ldots S_{s_{2}^{1}} L_{s_{1}^{1}}
\end{array}\right) .
$$

This is clearly a well-defined smooth function of $(E, \gamma)$. We need to check that $R_{s_{n-1}^{1}}$ is geometrically independent. Certainly $R_{s_{n-2}^{0}}$ is geometrically independent, since $s_{n-2}^{0} \in \tilde{\Gamma}^{n-2}$ and $\tilde{R} \in \mathcal{B}(\tilde{\Gamma})$. The parts of the above expression for $\mathbf{R}_{i}$ which depend on $\tilde{\mathbf{e}}_{0}, \ldots, \tilde{\mathbf{e}}_{n-2}$ describe a point on the codimension two hyperplane in $\mathbb{R}^{n}$ containing $R_{s_{n-2}^{0}}$. Thus $R_{s_{n-1}^{1}}$ is geometrically independent if and only if the sum of the squares of the last two components of the above column vector is positive, i.e. $S_{s_{n-1}^{1}}^{2} \ldots S_{s_{2}^{1}}^{2} L_{s_{1}^{1}}^{2}>0$. This follows from the fact that $\gamma \in \mathcal{D}_{P}\left(\Gamma^{*}\right)$. Thus, finally, the smooth map $\zeta$ is defined on all $\mathcal{P} \times \mathcal{D}_{P}\left(\Gamma^{*}\right)$ and takes values in $\mathcal{B}(\Gamma)$. Furthermore it is immediate that $\zeta$ is the inverse of $\eta$ : certainly this is true for $\tilde{\zeta}$ and $\tilde{\eta}$, but also in light of the theorem of section 3.3 the above expressions for the Cartesian coordinates of $\mathbf{R}_{i}$ in terms of $n$-dimensional spherical coordinates exactly invert our definitions of those coordinates in terms of Cartesian coordinates. This finishes the proof that $\eta$ is a diffeomorphism.

A smooth inverse to $\hat{\eta}$ can be found by composing $\zeta$ and the projection map $\rho$, and then factoring this through the projection $\pi_{2}: \mathcal{P} \times \mathcal{D}_{P}\left(\Gamma^{*}\right) \rightarrow \mathcal{D}_{P}\left(\Gamma^{*}\right)$. Thus $\hat{\eta}$ is also a diffeomorphism.

The formula $(*)$ explains why we describe our coordinates as "polyspherical" coordinates, for $\mathbf{R}_{i}$ is located relative to the pose $E_{r_{n}}(\tilde{R})$ using $n$-dimensional spherical coordinates, and there are many different poses used to position different points.

$\hat{\eta}$ is almost a chart $\phi$ in the manifold $G_{a} \| \mathcal{B}$; it remains only to order the coordinates and to choose a branch of the argument, such as $(-\pi, \pi)$, and then the resulting chart $\phi$ will have values in $\mathcal{D}_{P}(\phi)=(0, \infty)^{N-1} \times(-1,1)^{\sum_{k=2}^{n-1} N-k} \times$ $(-\pi, \pi)^{N-n} \subset \mathbb{R}^{n N-n(n+1) / 2}$. The domain of this chart is $\mathcal{D}_{C}(\phi)=\left\{\mathcal{O} \in \mathcal{D}_{C}(\Gamma) \mid\right.$ $\hat{Z}_{e^{*}}(\mathcal{O}) \neq-1$ for all $\left.e^{*} \in \Gamma_{*}^{n}\right\}$. We will loosely speak of $\hat{\eta}$ itself as being a coordinate chart. 
Note also that the orbit space $\mathcal{D}_{C}(\Gamma)$ itself acquires the structure of a principal fiber bundle with abelian structure group $\left(S^{1}\right)^{\Gamma_{*}^{n}}$, a fact which is not so obvious from the definition of $\mathcal{D}_{C}(\Gamma)$.

4.2. Scope of Z-system Coordinates. Thus our results so far provide us with a family, indexed by different Z-systems $\Gamma^{*}$, of smooth coordinate charts $\hat{\eta}$ on $\mathcal{D}_{C}(\Gamma)$. It remains to see if the domains of these charts cover all of $G_{a} \| \mathcal{B}$.

Theorem. Suppose $N \geq n \geq 2$ and $R \in \mathcal{B}$. Then there exists an $n$ dimensional unoriented Z-system $\Gamma$ on the set $\mathcal{N}$ such that $R \in \mathcal{B}(\Gamma)$, and hence $G_{a} R \in \mathcal{D}_{C}(\Gamma)$.

Proof. Without loss of generality let $\mathcal{N}=\{1,2, \ldots, N\}$. Without loss of generality assume that $s_{n}=\{1, \ldots, n\}$ and $\left\{\mathbf{R}_{1}, \ldots, \mathbf{R}_{n}\right\}$ is geometrically independent. We intend to define $\Gamma^{n-1}=\left\{s_{n}, s_{n+1}, \ldots, s_{N}\right\}$, and then extend it to an entire Zsystem; if $N=n$ then $\Gamma^{n-1}$ is now defined; otherwise we have only defined the first element of $\Gamma^{n-1}$.

If $s$ is any $n-1$ simplex such that $R_{s}$ is geometrically independent, and if for any $k$ simplex $t \subset s$ we denote by $\operatorname{span}\left(R_{t}\right)$ the $k$ dimensional affine subspace of $\mathbb{R}^{n}$ containing $R_{t}$ (and $\left.\operatorname{span}(\emptyset)=\emptyset\right)$, then we claim that $\operatorname{span}\left(R_{t_{1}}\right) \cap \operatorname{span}\left(R_{t_{2}}\right)=$ $\operatorname{span}\left(R_{t_{1} \cap t_{2}}\right)$. Clearly we have $\operatorname{span}\left(R_{t_{1} \cap t_{2}}\right) \subset \operatorname{span}\left(R_{t_{1}}\right) \cap \operatorname{span}\left(R_{t_{2}}\right)$. Now suppose $\tilde{\mathbf{R}} \in \operatorname{span}\left(R_{t_{1}}\right) \cap \operatorname{span}\left(R_{t_{2}}\right)$, i.e. $\left(\begin{array}{c}1 \\ \tilde{\mathbf{R}}\end{array}\right)=\sum_{i \in t_{1}} \alpha_{i}\left(\begin{array}{c}1 \\ \mathbf{R}_{i}\end{array}\right)=\sum_{i \in t_{2}} \beta_{i}\left(\begin{array}{c}1 \\ \mathbf{R}_{i}\end{array}\right)$. Then $\sum_{i \in t_{1} \backslash t_{2}} \alpha_{i}\left(\begin{array}{c}1 \\ \mathbf{R}_{i}\end{array}\right)+\sum_{i \in t_{1} \cap t_{2}}\left(\alpha_{i}-\beta_{i}\right)\left(\begin{array}{c}1 \\ \mathbf{R}_{i}\end{array}\right)-\sum_{i \in t_{2} \backslash t_{1}} \beta_{i}\left(\begin{array}{c}1 \\ \mathbf{R}_{i}\end{array}\right)=\left(\begin{array}{c}0 \\ \boldsymbol{\theta}\end{array}\right)$. Since $t_{1} \cup t_{2} \subset s$, the set $\left\{\left(\begin{array}{c}1 \\ \mathbf{R}_{i}\end{array}\right) \mid i \in t_{1} \cup t_{2}\right\}$ is linearly independent, and hence we have that $\alpha_{i}=0$ for $i \in t_{1} \backslash t_{2}, \beta_{i}=0$ for $i \in t_{2} \backslash t_{1}$, and $\alpha_{i}=\beta_{i}$ for $i \in t_{1} \cap t_{2}$. This implies that $\tilde{\mathbf{R}} \in \operatorname{span}\left(R_{t_{1} \cap t_{2}}\right)$, and hence the claim is established. An immediate consequence of the claim is that $\cap_{i \in s} \operatorname{span}\left(R_{s \backslash\{i\}}\right)=\emptyset$.

Suppose $N \geq n+1$ and $(n-1)$-simplices $s_{n}, s_{n+1}, \ldots, s_{N-1}$ have been defined such that $R_{s_{n}}, R_{s_{n+1}}, \ldots, R_{s_{N-1}}$ are all geometrically independent, and $\{j\}=s_{j} \backslash$ $s_{j-1}$ for all $j=n+1, \ldots, N-1$. Since $\cap_{i \in s_{N-1}} \operatorname{span}\left(R_{s_{N-1} \backslash\{i\}}\right)=\emptyset$ there is an $i \in s_{N-1}$ such that $\mathbf{R}_{N} \notin \operatorname{span}\left(R_{s_{N-1} \backslash\{i\}}\right)$. Thus define $s_{N}=\left(s_{N-1} \backslash\{i\}\right) \cup\{N\}$. Clearly $R_{s_{N}}$ is geometrically independent, and $\{N\}=s_{N} \backslash s_{N-1}$. Furthermore $s_{j} \cap\left(s_{l+1} \backslash s_{l}\right)=\emptyset$ for all $n \leq j \leq l \leq N-1$. Define $n$ simplices $\tau_{n+1}, \ldots, \tau_{N}$ by the rule: $\tau_{j}=s_{j-1} \cup s_{j}, j=n+1, \ldots, N$. Define $\Gamma^{n-1}=\left\{s_{n}, \ldots, s_{N}\right\}$ and $\Gamma^{n}=\left\{\tau_{n+1}, \ldots, \tau_{N}\right\} .\left(\Gamma^{n-1}, \Gamma^{n}\right)$ clearly defines a linear tree with $N-n$ edges.

We need to show that these can be extended to an unoriented Z-system $\Gamma$. Clearly, according to the definition of section $2.7,\left(s_{n}, \tau_{n+1}, s_{n+1}, \ldots, \tau_{N}, s_{N}\right)$ is an $n$-fence of length $N-n$. Using the lemma of section 2.7 we can inductively define a sequence of fences of decreasing orders and lengths. Let $\left(\Gamma_{j}^{j-1}, \Gamma_{j}^{j}\right)$ be derived from the fence of order $j$, where $\Gamma_{j}^{j-1}$ is the set of $(j-1)$-simplices and $\Gamma_{j}^{j}$ is the set of $j$-simplices. We will eventually define $\Gamma^{j}$ so that each of the $j$-simplices in these fences will be members of $\Gamma^{j}$ for $j=1, \ldots, n$. Let $\kappa$ denote the order of the last fence in this sequence and $l$ denote its length. We have either $\kappa=1$ or $l=0$ (or possibly both). If $\kappa=1, l>0$ then define $\mathcal{N}_{1}=\cup_{s \in \Gamma_{1}^{0}} s$, which has $l+1$ elements. In general if $l=0$, then $\Gamma_{\kappa}^{\kappa-1}$ has a single element, which we denote by $\mathcal{N}_{\kappa}$, and $\Gamma_{\kappa}^{\kappa}=\emptyset$. For example when $N=n$ we have $\kappa=n$ and $\Gamma^{n-1}=\Gamma_{n}^{n-1}=\left\{s_{n}\right\}$, where $\left(s_{n}\right)$ is an $n$-fence with length $l=0$. When $l=0, \kappa>1$ it is always possible 
to choose sets $\Gamma_{\kappa}^{0}, \Gamma_{\kappa}^{1}, \ldots, \Gamma_{\kappa}^{\kappa-2}$ such that $\left(\Gamma_{\kappa}^{1}, \ldots, \Gamma_{\kappa}^{\kappa-1}, \Gamma_{\kappa}^{\kappa}\right)$ is a $\kappa$-dimensional Zsystem on a set $\mathcal{N}_{\kappa}$ of $\kappa$ elements, where $\Gamma_{\kappa}^{0}=\left(\begin{array}{c}\mathcal{N}_{\kappa} \\ 1\end{array}\right)$ and $\Gamma_{\kappa}^{\kappa-1}=\left\{\mathcal{N}_{\kappa}\right\}$. (This can be done by choosing spanning trees in line graphs as in section 2.) Of course if $\kappa=1, l=0$ there is nothing to do. If $\kappa=2, l=0$ then $\mathcal{N}_{2}=\{a, b\}$ is a 1 -simplex, and $\Gamma_{2}^{0}=\{\{a\},\{b\}\}$. If $\kappa \geq 3, l=0$ then there are some choices to make.

Our strategy beyond this point is to inductively (for $k=\kappa, \ldots, n$ ) extend the sets $\Gamma_{k}^{0}, \Gamma_{k}^{1}, \ldots, \Gamma_{k}^{k}$ by adding leaves to each of the trees involved to obtain the sets $\Gamma_{k+1}^{0}, \Gamma_{k+1}^{1}, \ldots, \Gamma_{k+1}^{k}$. Then we adjoin the set $\Gamma_{k+1}^{k+1}$ to this list, so that the top level tree is a linear chain. We assume that $\mathcal{N}_{k}=\cup_{s \in \Gamma_{k}^{0}} s \subset\{1, \ldots, N\}$ and $\Gamma_{k}^{j} \subset\left(\begin{array}{c}\mathcal{N}_{k} \\ j+1\end{array}\right), j=0,1, \ldots, k$. We also assume that $\left(\Gamma_{k}^{j-1}, \Gamma_{k}^{j}\right)$ is a tree for $j=1, \ldots, k$ and whenever $s, s^{\prime} \in \Gamma_{k}^{j}$ are such that $s \cup s^{\prime} \in \Gamma_{k}^{j+1}$ then $s \cap s^{\prime} \in \Gamma_{k}^{j-1}$. This extension involves using information from the $k+1$-fence $\left(\Gamma_{k+1}^{k}, \Gamma_{k+1}^{k+1}\right)$. Let $\Gamma_{k+1}^{k}=\left\{s_{0}, s_{1}, \ldots, s_{m}\right\}$ and $\Gamma_{k+1}^{k+1}=\left\{\tau_{1}, \ldots, \tau_{m}\right\}$; we use all the notation introduced in section 2.7 to define from the $k+1$-fence $\left(s_{0}, \tau_{1}, s_{1}, \ldots, s_{m-1}, \tau_{m}, s_{m}\right)$ the $k$-fence $\left(t_{0}, s_{k_{1}-1}, t_{1}, \ldots, s_{k_{J}-1}, t_{J}\right)$. Thus $\Gamma_{k}^{k-1}=\left\{t_{0}, t_{1}, \ldots, t_{J}\right\}$ and $\Gamma_{k}^{k}=$ $\left\{s_{k_{1}-1}, \ldots, s_{k_{J}-1}\right\}$. Define also $k_{J+1}=m+2$. There is a mapping $g: \Gamma_{k+1}^{k} \backslash \Gamma_{k}^{k} \rightarrow$ $\Gamma_{k}^{k-1}$ defined as follows.

(1) $s_{0} \in \Gamma_{k+1}^{k} \backslash \Gamma_{k}^{k}$ and $g\left(s_{0}\right):=t_{0}=s_{0} \cap s_{1}$

(2) Also if $0 \leq j \leq J$ is such that $k_{j+1}-2 \geq k_{j}$ and $k_{j} \leq l \leq k_{j+1}-2$ then $s_{l} \in \Gamma_{k+1}^{k} \backslash \Gamma_{k}^{k}$, and $g\left(s_{l}\right):=t_{j}$.

If $s \in \Gamma_{k+1}^{k} \backslash \Gamma_{k}^{k}$ then we always have $t=g(s) \subset s$; let $s \backslash g(s)=\{i(s)\}$. We will be able to insure that we are always adding leaves provided we can show that the mapping $\Gamma_{k+1}^{k} \backslash \Gamma_{k}^{k} \rightarrow \mathcal{N}: s \mapsto i(s)$ is injective and its range is disjoint from $\mathcal{N}_{k}$.

This is a consequence of the disjointness of our fences. Suppose $s \in \Gamma_{k+1}^{k} \backslash \Gamma_{k}^{k}$ and $i(s) \in \mathcal{N}_{k}$. If $m=0$ then $\Gamma_{k}^{k-1}=\left\{\mathcal{N}_{k}\right\}$ and hence $g(s)=\mathcal{N}_{k}$, which is impossible since $i(s) \notin g(s)$. If $m>0$ there exists an $s^{\prime} \in \Gamma_{k}^{k}$ such that $i(s) \in s^{\prime}$ and which is as close to $s$ in the sequence $\left\{s_{0}, s_{1}, \ldots, s_{m}\right\}$ as possible. If $s^{\prime \prime}$ is adjacent to $s^{\prime}$ in the sequence $\left\{s_{0}, s_{1}, \ldots, s_{m}\right\}$ in the direction of $s$ then $i(s) \notin s^{\prime \prime}$ unless $s^{\prime \prime}=s$. If $s \neq s^{\prime \prime}$ then $i(s) \in s \cap\left(s^{\prime} \backslash s^{\prime \prime}\right)$, which contradicts our disjointness properties. If $s=s^{\prime \prime}$ then $s$ and $s^{\prime}$ are adjacent in the sequence $\left\{s_{0}, s_{1}, \ldots, s_{m}\right\}$ and since $s \in \Gamma_{k+1}^{k} \backslash \Gamma_{k}^{k}$ we have that $s \cap s^{\prime}$ must equal $g(s)$. Thus $i(s) \in g(s)$, which is impossible. Hence we cannot have $i(s) \in \mathcal{N}_{k}$.

Now suppose $s, s^{\prime} \in \Gamma_{k+1}^{k} \backslash \Gamma_{k}^{k}, s \neq s^{\prime}$, and $i(s)=i\left(s^{\prime}\right)$. If $s$ and $s^{\prime}$ are adjacent in the sequence $\left\{s_{0}, s_{1}, \ldots, s_{m}\right\}$ then $i(s)=i\left(s^{\prime}\right) \in s \cap s^{\prime}=g(s)=g\left(s^{\prime}\right)$, a contradiction. If $s$ and $s^{\prime}$ are not adjacent then let $s^{\prime \prime} \in \Gamma_{k+1}^{k}$ be adjacent to $s^{\prime}$ and between $s$ and $s^{\prime}$ in the sequence $\left\{s_{0}, s_{1}, \ldots, s_{m}\right\}$. Clearly $i\left(s^{\prime}\right) \notin s^{\prime \prime}$ because otherwise $i\left(s^{\prime}\right) \in s^{\prime} \cap s^{\prime \prime}=g\left(s^{\prime}\right)$, a contradiction. Therefore $i\left(s^{\prime}\right) \in s^{\prime} \backslash s^{\prime \prime}$, and hence $i(s)=i\left(s^{\prime}\right) \in s \cap\left(s^{\prime} \backslash s^{\prime \prime}\right)$, which contradicts our disjointness properties. Thus we must have $i(s) \neq i\left(s^{\prime}\right)$, which establishes the claimed injectivity.

So for any $s \in \Gamma_{k+1}^{k} \backslash \Gamma_{k}^{k}$ and $t^{k-1}(s):=g(s) \in \Gamma_{k}^{k-1}$ we can choose (in an arbitrary manner) a sequence $t^{j}(s) \in \Gamma_{k}^{j}$ for $j=0,1, \ldots, k-2$ such that $t^{0}(s) \subset$ $t^{1}(s) \subset \cdots \subset t^{k-1}(s)$. (Let us agree that $t^{-1}(s)=\emptyset$.) If $s \backslash t^{k-1}(s)=\{i(s)\}$ then $\{i(s)\} \subset t^{0}(s) \cup\{i(s)\} \subset \cdots \subset t^{k-2}(s) \cup\{i(s)\} \subset s$ is a sequence of simplices which are distinct from any in $\Gamma_{k}^{j}, j=0,1, \ldots, k$. Thus we define

$$
\Gamma_{k+1}^{j}=\Gamma_{k}^{j} \cup\left\{t^{j-1}(s) \cup\{i(s)\} \mid s \in \Gamma_{k+1}^{k} \backslash \Gamma_{k}^{k}\right\}, \quad 0 \leq j \leq k-1,
$$




$$
\mathcal{N}_{k+1}=\mathcal{N}_{k} \cup\left\{i(s) \mid s \in \Gamma_{k+1}^{k} \backslash \Gamma_{k}^{k}\right\} .
$$

When the new tree $\left(\Gamma_{k+1}^{j-1}, \Gamma_{k+1}^{j}\right)$ is compared with the old tree $\left(\Gamma_{k}^{j-1}, \Gamma_{k}^{j}\right)$ we see for each $s$ that a new vertex $t^{j-2}(s) \cup\{i(s)\}$ has been added, and a new edge $t^{j-1}(s) \cup\{i(s)\}$ has also been added connecting the new vertex to the old vertex $t^{j-1}(s)$. Thus the addition of these new simplices are in every case the addition of leaves, with the consequence that after these additions we have trees at each level. Since $\left(t^{j-2}(s) \cup\{i(s)\}\right) \cap t^{j-1}(s)=t^{j-2}(s)$, the intersection condition is satisfied. Hence $\Gamma_{k+1}^{0}, \ldots, \Gamma_{k+1}^{k+1}$ is an extended system of sets satisfying the same hypotheses that $\Gamma_{k}^{0}, \ldots, \Gamma_{k}^{k}$ satisfied.

Thus we ascend the hierarchy of fences, using each one as the basis of an extension as we have described. When completed we have the sets $\Gamma_{n}^{0}, \Gamma_{n}^{1}, \ldots, \Gamma_{n}^{n}$, which is an unoriented Z-system on the set $\mathcal{N}_{n}$. Since $\Gamma_{n}^{n-1}=\Gamma^{n-1}$ and $\Gamma_{n}^{n}=\Gamma^{n}$, by the lemma of section 2.6 we must have $\mathcal{N}_{n}=\{1, \ldots, N\}$. Clearly then we have defined a Z-system $\Gamma$ such that $R \in \mathcal{B}(\Gamma)$.

Thus Z-systems index coordinate charts in an atlas which covers the manifold $G_{a} \backslash \mathcal{B}$, over which $\mathcal{B}$ is a principal bundle with structure group $G_{a}$. For other mathematical results on the principal bundle $\rho: \mathcal{B} \rightarrow G_{a} \Downarrow \mathcal{B}$ see [30] (where $G_{a}=$ $\left.\mathbb{R}^{n} \times \mathrm{O}(n)\right)$, and [50].

\section{Z-MATRICES}

5.1. Unlabeled Z-matrices Defined. Three dimensional Z-matrices were discussed in the introduction. Here we give a rigorous mathematical definition of labeled and unlabeled Z-matrices in the $n$-dimensional case.

Definition. An (unlabeled) $n$-dimensional $Z$-matrix on the set $\mathcal{N}$ is a mapping

$$
\alpha:\left\{(i, j) \in \mathbb{Z}^{2} \mid 0 \leq j \leq n, j<i \leq N\right\} \rightarrow \mathcal{N},
$$

where $N=|\mathcal{N}| \geq n$, with the following properties:

(1) $\mathcal{N}=\{\alpha(i, 0) \mid 1 \leq i \leq N\}$.

(2) For every $1 \leq k \leq n$ and for every $k<i \leq N$ there exists $k \leq i^{\prime}<i$ such that $\{\alpha(i, j) \mid 1 \leq j \leq k\}=\left\{\alpha\left(i^{\prime}, j\right) \mid 0 \leq j \leq k-1\right\}$.

The precise mathematical definition of the $n$-dimensional Z-matrix is apparently new to this work. For a different looking definition in the 3 dimensional case, see [70]. A different use of the term Z-matrix is in [75]. It may be that some of the conditions in the above definition can be omitted without changing the concept; we have not attempted a minimal axiomatization of Z-matrices.

An example of an unlabeled 3-dimensional Z-matrix on the set $\mathcal{N}=\left\{C, O, H_{1}\right.$, $\left.H_{2}, H_{3}, H\right\}$ is as follows.

$$
\begin{array}{lcccc} 
& j=0 & j=1 & j=2 & j=3 \\
i=1 & C & & & \\
i=2 & O & C & & \\
i=3 & H_{1} & C & O & \\
i=4 & H_{2} & C & O & H_{1} \\
i=5 & H_{3} & C & O & H_{1} \\
i=6 & H & O & C & H_{1}
\end{array}
$$


This (unlabeled) Z-matrix is for the molecule methanol, so refer to Figures 2 and 3. A labeled form (precisely defined below) of this Z-matrix was listed in the introduction.

5.2. Relating Z-matrices and Z-systems. The question naturally arises about the exact relation between Z-matrices and Z-systems.

Theorem. Let $Z$ mat $(\mathcal{N})$ denote the set of all (unlabeled) Z-matrices $\alpha$ on $\mathcal{N}$. Let Zsys $(\mathcal{N})$ denote the set of all triples $(\Gamma, r, \lambda)$, where $\Gamma$ is an unoriented $Z$ system on $\mathcal{N}, r$ is a site from $\Gamma$, and $\lambda$ is a leaf-picking order in the rooted tree $\left(\Gamma^{n-1}, \Gamma^{n}, s_{n-1}(r)\right)$. Define a mapping $\Psi: \operatorname{Zmat}(\mathcal{N}) \rightarrow \operatorname{Zsys}(\mathcal{N}): \alpha \mapsto(\Gamma, r, \lambda)$, where

$$
\begin{aligned}
\Gamma^{k} & =\{\{\alpha(i, j) \mid 0 \leq j \leq k\} \mid k+1 \leq i \leq N\}, \quad 1 \leq k \leq n, \\
r & =(\alpha(1,0), \alpha(2,0), \ldots, \alpha(n, 0)), \\
\lambda & =\left(v_{n}, \ldots, v_{N}\right) \text { is an ordering of } \Gamma^{n-1}, \text { where } \\
& v_{i}=\{\alpha(i, j) \mid 0 \leq j \leq n-1\}, \quad n \leq i \leq N .
\end{aligned}
$$

Then $\Psi$ is a bijection.

Proof. Assume that $\alpha$ is a Z-matrix and $\Psi(\alpha)=(\Gamma, r, \lambda)$. To demonstrate that $\Gamma$ is an unoriented Z-system we first verify the following: for all $1 \leq j \leq n$ and for all $j<i \leq N$ there exists $1 \leq i^{\prime}<i$ such that $\alpha(i, j)=\alpha\left(i^{\prime}, 0\right)$. This result is a consequence of condition (2) if $j=1$, so suppose it is true for $1 \leq j \leq j^{\prime}-1 \leq n-1$. Since by condition $(2)\left\{\alpha(i, 1), \ldots, \alpha\left(i, j^{\prime}\right)\right\}=\left\{\alpha\left(i^{\prime}, 0\right), \ldots, \alpha\left(i^{\prime}, j^{\prime}-1\right)\right\}$ we can apply the induction hypothesis to obtain $1 \leq i^{\prime \prime}<i^{\prime}$ such that $\alpha\left(i, j^{\prime}\right)=\alpha\left(i^{\prime \prime}, 0\right)$. Thus the result is true for $j=j^{\prime}$ as well.

Next we must show that $\{\alpha(i, j) \mid 0 \leq j \leq k\}$ is always an abstract $k$-simplex, i.e. it cannot have fewer than $k+1$ elements. Let $2 \leq i \leq N$ be the first row in which repetitions occur (assuming by way of contradiction that they occur somewhere). Let $j \geq 0$ be as small as possible such that $\alpha(i, j)=\alpha(i, k)$ for some $j<k \leq n$. If $j=0$ then since $k \geq 1$ we must have $\alpha(i, k) \in\left\{\alpha\left(i^{\prime}, 0\right) \mid 0 \leq i^{\prime}<i\right\}$, a contradiction with condition (1). If $j \geq 1$ then by condition $(2)\{\alpha(i, 1), \ldots, \alpha(i, k)\}=$ $\left\{\alpha\left(i^{\prime}, 0\right), \ldots, \alpha\left(i^{\prime}, k-1\right)\right\}$ for some $i^{\prime}<i$, and a repetition must have occurred on an earlier row, a contradiction of the choice of $i$.

Now suppose $\{\alpha(i, j) \mid 0 \leq j \leq k\} \in \Gamma^{k}$ is a $k$-simplex. It is incident on two $(k-1)$-simplices, namely $\{\alpha(i, j) \mid 0 \leq j \leq k-1\}$ and $\{\alpha(i, j) \mid 1 \leq j \leq k\}$. We need to show that it is incident on no other $(k-1)$-simplex. Suppose $s \in \Gamma^{k-1}$ is a $(k-1)$-simplex on which $\{\alpha(i, j) \mid 0 \leq j \leq k\}$ is incident. We have $s=$ $\left\{\alpha\left(i^{\prime}, j\right) \mid 0 \leq j \leq k-1\right\}$ for some $k \leq i^{\prime} \leq N . i^{\prime}>i$ is impossible since then $\alpha\left(i^{\prime}, 0\right)=\alpha\left(i^{\prime \prime}, 0\right)$ for some $i^{\prime \prime} \leq i<i^{\prime}$ contradicting condition (1). If $i^{\prime}=i$ then $s$ is one of the two $(k-1)$-simplices we already know about. So suppose $i^{\prime}<i$. $s$ is obtained from $\{\alpha(i, j) \mid 0 \leq j \leq k\}$ by omitting a single element, which must be $\alpha(i, 0)$ since otherwise $\alpha(i, 0) \in s$ and hence $\alpha(i, 0)=\alpha\left(i^{\prime \prime}, 0\right)$ for some $i^{\prime \prime} \leq i^{\prime}<i$, contradicting condition (1). Thus $s=\{\alpha(i, j) \mid 1 \leq j \leq k\}$, which is the other of the $(k-1)$-simplices that we already knew about.

Since each $k$-simplex is incident on exactly two $(k-1)$-simplices, $\left(\Gamma^{k-1}, \Gamma^{k}\right)$ is a graph. It is easy to see that this graph is connected, since every $(k-1)$ simplex (vertex) appears on a row of the Z-matrix defining a $k$-simplex (edge) connecting it to a $(k-1)$-simplex (vertex) appearing on a previous row. This 
can only end if the previous vertex is the first $(k-1)$-simplex specified, namely $\{\alpha(k-1,0), \ldots, \alpha(k-1, k-2)\}$. A connected graph with one fewer edges than vertices must be a tree. Finally it is clear that the intersection of the two $(k-1)$ simplices $\{\alpha(i, j) \mid 0 \leq j \leq k-1\}$ and $\{\alpha(i, j) \mid 1 \leq j \leq k\}$ is the $(k-2)$-simplex $\{\alpha(i, j) \mid 1 \leq j \leq k-1\} \in \Gamma^{k-2}$ by condition (2). Thus $\Gamma=\left(\Gamma^{1}, \ldots, \Gamma_{*}^{n}\right)$ is an unoriented Z-system.

We claim that $r=(\alpha(1,0), \alpha(2,0), \ldots, \alpha(n, 0))$ is a site for the Z-system $\Gamma$. Clearly $\{\alpha(1,0), \alpha(2,0)\}=\{\alpha(2,0), \alpha(2,1)\} \in \Gamma^{1}$. Suppose $\{\alpha(i, 0) \mid 1 \leq i \leq k\}=$ $\{\alpha(k, j) \mid 0 \leq j \leq k-1\}$ for some $2 \leq k<n$. Then $\{\alpha(k+1, j) \mid 1 \leq j \leq k\}$ must equal $\{\alpha(k, j) \mid 0 \leq j \leq k-1\}$ by condition (2), and by the induction hypothesis this must equal $\{\alpha(i, 0) \mid 0 \leq i \leq k\}$. Adding the element $\alpha(k+1,0)$ to both sets shows that $\{\alpha(i, 0) \mid 1 \leq i \leq k+1\}=\{\alpha(k+1, j) \mid 0 \leq j \leq k\} \in \Gamma^{k}$. Thus $r$ is a site of $\Gamma$.

Note that $\{\alpha(N, j) \mid 0 \leq j \leq n-1\} \in \Gamma^{n-1}$ is of degree one in the graph $\left(\Gamma^{n-1}, \Gamma^{n}, \sigma\right)$, since no $n$-simplex other than $\{\alpha(N, j) \mid 0 \leq j \leq n\} \in \Gamma^{n}$ can contain the element $\alpha(N, 0)$. Thus the Z-matrix determines a leaf-picking order for the rooted tree $\left(\Gamma^{n-1}, \Gamma^{n}, \sigma\right)$, in the sense that the vertices of this tree are ordered (assigned numbers $n$ through $N$ ) starting at the root vertex $s_{n-1}(r)=$ $\{\alpha(1,0), \ldots, \alpha(n, 0)\}=\{\alpha(n, 0), \ldots, \alpha(n, n-1)\}$ with number $n$, such that for every $n<k \leq N$ the vertex $\{\alpha(k, 0), \ldots, \alpha(k, n-1)\}$ is a leaf of the tree subgraph of $\left(\Gamma^{n-1}, \Gamma^{n}\right)$ induced by the set of vertices numbered $n$ through $k$. Thus the mapping $\Psi$ is well-defined.

Now we will show that $\Psi$ is bijective, namely given $(\Gamma, r, \lambda) \in \operatorname{Zsys}(\mathcal{N})$ there is a unique Z-matrix $\alpha$ such that $\Psi(\alpha)=(\Gamma, r, \lambda)$. The key ideas for how the Z-matrix is determined have already been explained in the proof of the main theorem in section 4.1. We obviously must define $\alpha(1,0), \ldots, \alpha(n, 0)$ so that the root site is given by $r=(\alpha(1,0), \alpha(2,0), \ldots, \alpha(n, 0))$. Also we must define $\alpha(2,1)=$ $\alpha(1,0)$, so that $\{\alpha(2,0), \alpha(2,1)\}=s_{1}(r) \in \Gamma^{1}$. If $S \subset \Gamma^{0}$ then define the notation: $\Gamma_{S}^{k}=\left\{s \in \Gamma^{k} \mid s \subset S\right\}$. Notice that $\Gamma_{s_{1}(r)}^{0}=\{\{\alpha(1,0)\},\{\alpha(2,0)\}\}$ and $\Gamma_{s_{1}(r)}^{1}=\{\{\alpha(2,0), \alpha(2,1)\}\}$. Now suppose the first $k$ rows of the Z-matrix have been defined for $2 \leq k \leq n-1$ such that for all $0 \leq h \leq k-1$ we have $\Gamma_{s_{k-1}(r)}^{h}=\{\{\alpha(i, j) \mid 0 \leq \bar{j} \leq h\} \mid h+1 \leq i \leq k\}$.

$$
\begin{aligned}
& s_{0}^{0}=\{\alpha(k+1,1)\} \\
& s_{1}^{0}=\quad\{\alpha(k+1,1), \quad \alpha(k+1,2)\} \\
& \begin{array}{ccccc}
\vdots & \vdots & \vdots & \ddots & \\
s_{k-1}^{0}= & \{\alpha(k+1,1), & \alpha(k+1,2), & \cdots & \alpha(k+1, k)\}
\end{array} \\
& s_{k}^{1}=\{\alpha(k+1,0), \quad \alpha(k+1,1), \quad \alpha(k+1,2), \quad \ldots \quad \alpha(k+1, k)\} \\
& \begin{array}{ccc}
\vdots & \vdots & \vdots
\end{array} \\
& s_{2}^{1}=\{\alpha(k+1,0), \quad \alpha(k+1,1), \quad \alpha(k+1,2)\} \\
& s_{1}^{1}=\{\alpha(k+1,0), \quad \alpha(k+1,1)\} \\
& s_{0}^{1}=\{\alpha(k+1,0)\}
\end{aligned}
$$

Define $s_{k}^{1}=s_{k}(r)=\{\alpha(1,0), \ldots, \alpha(k+1,0)\} \in \Gamma^{k} . s_{k}^{1}$ is incident on two $(k-1)$ simplices, one of which is $s_{k-1}^{0}=s_{k-1}(r)=\{\alpha(1,0), \ldots, \alpha(k, 0)\} \in \Gamma^{k-1}$, and the other we denote by $s_{k-1}^{1}$. Note that $\alpha(k+1,0) \in s_{k-1}^{1}$. As in the proof of the main theorem we define $s_{k-2}^{0}=s_{k-1}^{0} \cap s_{k-1}^{1}$, and let $s_{k-2}^{1}$ be the $(k-2)$ simplex on which $s_{k-1}^{1}$ is incident besides $s_{k-2}^{0}$. Note again that $\alpha(k+1,0) \in s_{k-2}^{1}$. 
Continuing this procedure as in the proof of the main theorem we end up with two 0-simplices: $s_{0}^{1}=\{\alpha(k+1,0)\}$ and $s_{0}^{0}$. We define $\alpha(k+1,1), \ldots, \alpha(k+1, k)$ such that $s_{h}^{0}=\{\alpha(k+1,1), \ldots, \alpha(k+1, h+1)\}$ for $h=0,1, \ldots, k-1$. Since $s_{0}^{0} \subset s_{1}^{0} \subset \cdots \subset s_{k-1}^{0}=s_{k-1}(r)$ we have that $s_{h}^{0} \in \Gamma_{s_{k-1}(r)}^{h}$ for $h=0,1, \ldots, k-1$, and by the induction hypothesis this means that $s_{h}^{0}=\{\alpha(i, j) \mid 0 \leq j \leq h\}$ for some $h+1 \leq i<k+1$, verifying condition (2) for row $k+1$ of the Z-matrix. Note also that $s_{h}^{1}=\{\alpha(k+1,0), \alpha(k+1,1), \ldots, \alpha(k+1, h)\}$ for $h=0,1, \ldots, k$. Thus $\Gamma_{s_{k-1}(r)}^{h} \cup\left\{s_{h}^{1}\right\} \subset \Gamma_{s_{k}(r)}^{h}$. Thus these two sets will be equal if we can show that $\left|\Gamma_{s_{k}(r)}^{h}\right|=k-h+1$, since $\left|\Gamma_{s_{k-1}(r)}^{h}\right|=k-h$. As we argued in the proof of the second lemma in section $2\left(\Gamma_{s_{k}(r)}^{l-1}, \Gamma_{s_{k}(r)}^{l}\right)$ is an acyclic subgraph of the tree $\left(\Gamma^{l-1}, \Gamma^{l}, \sigma\right)$, and hence $\left|\Gamma_{s_{k}(r)}^{l-1}\right| \geq\left|\Gamma_{s_{k}(r)}^{l}\right|+1$. Since $\Gamma_{s_{k}(r)}^{k}=\left\{s_{k}^{1}\right\}$, and hence $\left|\Gamma_{s_{k}(r)}^{k}\right|=1$, we have $\left|\Gamma_{s_{k}(r)}^{l}\right| \geq k-l+1$ for all $l=0,1, \ldots, k$. But then $k+1 \leq\left|\Gamma_{s_{k}(r)}^{0}\right| \leq\left|s_{k}(r)\right|=k+1$. Thus $k-l+1 \leq\left|\Gamma_{s_{k}(r)}^{l}\right| \leq k-l+1$ for $l=0,1, \ldots, k$, showing the desired result. This shows that $\Gamma_{s_{k}(r)}^{h}=\{\{\alpha(i, j) \mid 0 \leq j \leq h\} \mid h+1 \leq i \leq k+1\}$ for $h=0,1, \ldots, k$. This finishes the induction step, so that the first $n$ rows of the Z-matrix $\alpha$ are defined so that condition (2) is satisfied for these rows and moreover we have $\Gamma_{s_{n-1}(r)}^{h}=\{\{\alpha(i, j) \mid 0 \leq j \leq h\} \mid h+1 \leq i \leq n\}$ for $h=0,1, \ldots, n-1$.

To define rows $n+1$ through $N$ of the Z-matrix we need to use the leaf-picking order $\lambda$ for the tree $\left(\Gamma^{n-1}, \Gamma^{n}\right)$ with root $s_{n-1}(r)$. Thus the elements of $\Gamma^{n-1}$ are assumed numbered $n$ through $N$, the root vertex being numbered $n$, so that for all $n \leq k \leq N$ the vertex numbered $k$ is a leaf in the subgraph of $\left(\Gamma^{n-1}, \Gamma^{n}\right)$ induced by the set of vertices numbered $n$ through $k$. Suppose rows 1 through $k$ of the Z-matrix have been defined, where $n \leq k \leq N-1$, such that $\Gamma_{S(k)}^{h}=\{\{\alpha(i, j) \mid$ $0 \leq j \leq h\} \mid h+1 \leq i \leq k\}$ for $h=0,1, \ldots, n$, where $S(k)=\{\alpha(1,0), \ldots, \alpha(k, 0)\}$. Assume that condition (2) is satisfied for each of these $k$ rows. Also assume that for all $n \leq i \leq k$ that $\{\alpha(i, 0), \ldots, \alpha(i, n-1)\}$ is element number $i$ in $\Gamma^{n-1}$. Let $s_{n-1}^{1}$ be element number $k+1$ in $\Gamma^{n-1}$, and since it is a leaf in the subgraph of $\left(\Gamma^{n-1}, \Gamma^{n}\right)$ induced by the set of vertices numbered $n$ through $k+1$, let the unique edge of this subgraph incident on $s_{n-1}^{1}$ be denoted by $s_{n}^{1} \in \Gamma^{n}$. Let $s_{n-1}^{0} \in \Gamma^{n-1}$ denote the other vertex on which this edge is incident. It must be one of the vertices numbered $n$ through $k$, and hence in $\Gamma_{S(k)}^{n-1}$. By the properties of Z-systems we have that $s_{n-2}^{0}=s_{n-1}^{0} \cap s_{n-1}^{1} \in \Gamma^{n-2}$. Also let $s_{n-2}^{1} \in \Gamma^{n-2}$ denote the other vertex besides $s_{n-2}^{0}$ on which the edge $s_{n-1}^{1}$ is incident. Continuing as in the proof of the main theorem we define $s_{h}^{1}$ and $s_{h}^{0}$ for $h=0,1, \ldots, n-1$. We define $\alpha(k+1,0)$ such that $s_{0}^{1}=\{\alpha(k+1,0)\}$, and we define $\alpha(k+1,1), \ldots, \alpha(k+1, n)$ such that $s_{h}^{0}=\{\alpha(k+1, j) \mid 1 \leq j \leq h+1\}$ for $h=0,1, \ldots, n-1$. We also have that $s_{h}^{1}=\{\alpha(k+1, j) \mid 0 \leq j \leq h\}$ for $h=0,1, \ldots, n$. We know that $\left|\Gamma_{S(k)}^{n}\right|=k-n$ by the induction hypothesis. $s_{n}^{1} \in \Gamma_{S(k+1)}^{n}$ is incident on element number $k+1$ of $\Gamma^{n-1}$, and hence is distinct from the edges in $\Gamma_{S(k)}^{n}$ which are incident on elements numbered $n$ through $k$ of $\Gamma^{n-1}$; so $\left|\Gamma_{S(k+1)}^{n}\right| \geq k-n+1$. Using the fact that $\left(\Gamma_{S}^{h-1}, \Gamma_{S}^{h}\right)$ is a subgraph of a tree, with the consequence that $\left|\Gamma_{S}^{h-1}\right| \geq\left|\Gamma_{S}^{h}\right|+1$, we obtain after $n$ applications that $\left|\Gamma_{S(k+1)}^{0}\right| \geq k+1$. Thus $|S(k+1)|=k+1$ and hence $\alpha(k+1,0) \notin S(k)$. Furthermore we have that $\left|\Gamma_{S(k+1)}^{h}\right|=k-h+1$, for $h=0,1, \ldots, n$. Thus $\Gamma_{S(k+1)}^{h}=\Gamma_{S(k)}^{h} \cup\left\{s_{h}^{1}\right\}$ for $h=0,1, \ldots, n$. Since $s_{h}^{0} \in \Gamma_{S(k)}^{h}$ for $0 \leq h \leq n-1$ we have that condition (2) is satisfied by row $k+1$ of the Z-matrix. 
This finishes the inductive definition of the Z-matrix. The first column of the Zmatrix has $N$ distinct elements from the set $\mathcal{N}$, which has exactly $N$ elements; so condition (1) is also satisfied.

The above construction of the Z-matrix $\alpha$ obviously satisfies $\Psi(\alpha)=(\Gamma, r, \lambda)$. Also if $\tilde{\alpha}$ is another Z-matrix and $\Psi(\tilde{\alpha})=\Psi(\alpha)=(\Gamma, r, \lambda)$, then the Z-matrices $\alpha$ and $\tilde{\alpha}$ must coincide by a simple induction argument, similar to those already given; the reader is invited to generate this argument as an exercise.

Any Z-matrix $\alpha$ on $\mathcal{N}$ determines an unoriented Z-system $\Gamma$ such that $\Psi(\alpha)=$ $(\Gamma, r, \lambda)$. But it also determines an oriented Z-system $\Gamma^{*}(\alpha)$, whose underlying unoriented Z-system is $\Gamma$, by the rule

$$
\Gamma_{*}^{n}(\alpha)=\{[\alpha(i, 0), \alpha(i, 1), \ldots, \alpha(i, n)] \mid n<i \leq N\} .
$$

Not every possible set $\Gamma_{*}^{n}$ of oriented $n$-simplices such that $\Upsilon$ is a bijection of $\Gamma_{*}^{n}$ onto $\Gamma^{n}$ is equal to $\Gamma_{*}^{n}(\alpha)$, where $\alpha=\Psi^{-1}(\Gamma, r, \lambda)$ for some site $r$ from $\Gamma$ and leaf-picking order $\lambda$ for $(\Gamma, r)$. For example, suppose $\Gamma$ is the underlying unoriented Z-system for methanol given in Figure 2. Choose the root site $r=\left(C, O, H_{1}\right)$, and the leaf-picking order $\lambda=\left(\left\{O, C, H_{1}\right\},\left\{O, C, H_{2}\right\},\left\{O, C, H_{3}\right\},\{O, C, H\}\right)$. Then $\alpha=\Psi^{-1}(\Gamma, r, \lambda)$ is the same as the Z-matrix $\alpha$ given above for methanol. We can make the following choice of $\Gamma_{*}^{3}$ and compare it with the Z-system determined by $\alpha$ :

$$
\begin{aligned}
\Gamma_{*}^{3} & =\left\{\left[C, O, H_{1}, H_{2}\right],\left[C, O, H_{1}, H_{3}\right],\left[H, O, C, H_{1}\right]\right\}, \\
\Gamma_{*}^{3}(\alpha) & =\left\{\left[H_{2}, C, O, H_{1}\right],\left[H_{3}, C, O, H_{1}\right],\left[H, O, C, H_{1}\right]\right\} .
\end{aligned}
$$

Since $\left[H_{j}, C, O, H_{1}\right]=\left[C, O, H_{j}, H_{1}\right]=-\left[C, O, H_{1}, H_{j}\right], j=2,3$, we see that $\Gamma_{*}^{3}(\alpha) \neq \Gamma_{*}^{3}$. All the improper (c.f. section 6.2) edges of the tree $\left(\Gamma^{2}, \Gamma^{3}\right)$ are always directed toward the root vertex $s_{2}(r)$ by the Z-matrix convention for any leaf-picking order. This makes $\Gamma_{*}^{3}$ unattainable regardless of the choice of the root site.

5.3. Labeled Z-matrices. The concept of a labeled Z-system has its counterpart in the concept of a labeled Z-matrix.

Definition. If $\alpha:\left\{(i, j) \in \mathbb{Z}^{2} \mid 0 \leq j \leq n, j<i \leq N\right\} \rightarrow \mathcal{N}$ is a Z-matrix, then a mapping $\beta:\left\{(i, j) \in \mathbb{Z}^{2} \mid 1 \leq j \leq n, j<i \leq N\right\} \rightarrow \mathbb{R}$ is a labeling of the Z-matrix $\alpha$ if

(1) $\beta(i, 1)>0$ for $2 \leq i \leq N$;

(2) $0<\beta(i, j)<\pi$ for $2 \leq j \leq n-1, j+1 \leq i \leq N$;

(3) $-\pi<\beta(i, n) \leq \pi$ for $n+1 \leq i \leq N$.

Suppose $\gamma \in \mathcal{D}_{P}\left(\Gamma^{*}\right)$ is a labeling for the Z-system $\Gamma^{*}$, and suppose $\alpha=\Psi(\Gamma, r, \lambda)$ for some choice of root site $r$ and leaf-picking order $\lambda$. Assume that $\Gamma_{*}^{n}=\Gamma_{*}^{n}(\alpha)$. Then we define the labeling $\beta$ of the Z-matrix associated to $\gamma$ as follows.

(1) If $2 \leq i \leq N$ then define $\beta(i, 1)=L_{e}$, where $e=\{\alpha(i, 0), \alpha(i, 1)\}$.

(2) If $2 \leq j \leq n-1$ and $j+1 \leq i \leq N$ then define $\beta(i, j)=\cos ^{-1} C_{e}$, where $e=\{\alpha(i, h) \mid 0 \leq h \leq j\}$.

(3) If $n+1 \leq k \leq N$ then define $\beta(k, n) \in(-\pi, \pi]$ such that $e^{i(-1)^{n-1} \beta(k, n)}=$ $Z_{e^{*}}$, where $e^{*}=[\alpha(k, 0), \ldots, \alpha(k, n)]$. 


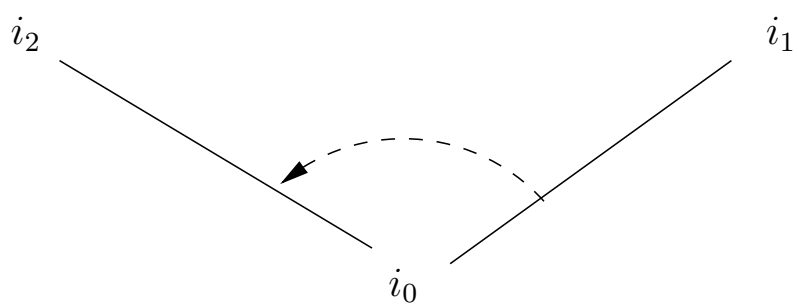

Figure 7. An oriented angle in the case $n=2$. The Z-system convention would assign a positive angle to $\left[i_{0}, i_{1}, i_{2}\right]$. The Z-matrix convention would assign a positive angle to $\left[i_{1}, i_{0}, i_{2}\right]$.

The only unexpected aspect of this definition is the factor $(-1)^{n-1}$ appearing in the equation for $\beta(k, n)$. It reflects a discrepancy in even dimensional spaces between the interpretation of an oriented $n$ simplex that is natural for Z-systems versus that which is conventional and natural for Z-matrices (see Figure 7). In Z-systems it is natural to interpret $\left[i_{0}, \ldots, i_{n}\right]$ in terms of a positive rotation of the halfhyperplane bounded by the codimension 2 subset $Y$ (spanned by $\mathbf{R}_{i_{0}}, \ldots, \mathbf{R}_{i_{n-2}}$ ) and containing $\mathbf{R}_{i_{n-1}}$ into the half-hyperplane bounded by $Y$ and containing $\mathbf{R}_{i_{n}}$. According to the usual conventions for Z-matrices this would be represented by the oriented $n$ simplex $\left[i_{n-1}, i_{0}, \ldots, i_{n-2}, i_{n}\right]$. These two oriented $n$ simplices are related by $n-1$ transpositions. Fortunately in 3 dimensions, where the Z-matrix convention is standard among chemists, the two conventions agree. Note that since $Z_{-e^{*}}(R)=\overline{Z_{e^{*}}(R)}$ we may relax the assumption $\Gamma_{*}^{n}=\Gamma_{*}^{n}(\alpha)$ in the above by introducing a negative sign in the Z-matrix label $\beta(k, n)$ when the orientation from $\Gamma_{*}^{n}$ is opposite to that from the Z-matrix.

Labelled Z-matrices are very close to usual coordinate systems where the coordinates are all assumed to be real numbers and where a specific ordering of the coordinates is specified. However, even a labelled Z-matrix does not tell us whether the coordinates should be ordered by rows or by columns. In our discussion of Z-systems we have not assigned any importance to the ordering of coordinates.

\section{Biomolecular Shape}

6.1. Terminology for Molecular Z-systems. In this section we will not always distinguish notationally between a Z-system $\Gamma^{*}$ and its underlying unoriented Zsystem $\Gamma$. We begin by explaining some terminology natural in the context of molecules. Recall from the Introduction that members of the sets $\Gamma^{0}, \Gamma^{1}, \Gamma^{2}, \Gamma^{3}$ are called atoms, bonds, triangles and tetrahedra respectively. The bonds in $\Gamma^{1}$ should not be confused with chemical bonds, although it is often convenient to choose as bonds in $\Gamma^{1}$ pairs of atom names which are chemically bonded in the molecule. We will see (in our discussion of five-membered rings) that it is sometimes convenient to include as an element in $\Gamma^{1}$ a pair of atom names corresponding to atoms which are not covalently bonded in the molecule. We call a pair $\alpha=\left\{b_{1}, b_{2}\right\}$ of bonds, where $b_{1} \cup b_{2} \in \Gamma^{2}$ and $b_{1} \cap b_{2} \in \Gamma^{0}$, an angle of $\Gamma$. Recall the lemma from section 2.6 implies that triangles in $\Gamma^{2}$ and angles of $\Gamma$ are in one-to-one correspondence, where the triangle $t$ associated to the angle $\left\{b_{1}, b_{2}\right\}$ is of course $t=b_{1} \cup b_{2}$. The common atom of the angle $\left\{b_{1}, b_{2}\right\}$ is $a=b_{1} \cap b_{2}$. We call a pair $\omega=\left\{t_{1}, t_{2}\right\}$ of triangles, where $t_{1} \cup t_{2} \in \Gamma^{3}$ and $t_{1} \cap t_{2} \in \Gamma^{1}$, a wedge of $\Gamma$. Similarly this same lemma implies 
a)

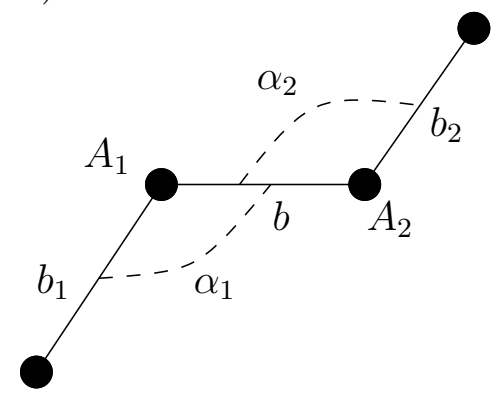

b) $\quad A_{1}=A_{2}$

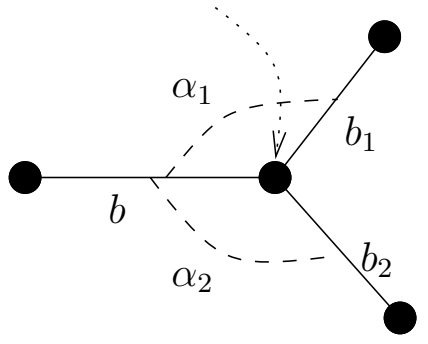

Figure 8. (a) $\omega=\left\{t_{1}, t_{2}\right\}$ is a dihedral. (b) $\omega=\left\{t_{1}, t_{2}\right\}$ is an improper. In both cases angles $\alpha_{1}, \alpha_{2}$ correspond to triangles $t_{1}, t_{2}$, and $b=t_{1} \cap t_{2}$ is the common bond shared by $t_{1}, t_{2}$.

that tetrahedra in $\Gamma^{3}$ and wedges of $\Gamma$ are in one-to-one correspondence, where the tetrahedron $d$ associated to the wedge $\left\{t_{1}, t_{2}\right\}$ is $d=t_{1} \cup t_{2}$. The common bond of the wedge $\left\{t_{1}, t_{2}\right\}$ is $b=t_{1} \cap t_{2}$.

6.2. Dihedrals and Impropers. According to the lemma from section 2.6 the only bonds which are subsets of a triangle are those in the associated angle. Hence the common bond $b$ of a wedge must be part of both of the angles associated to $t_{1}$ and $t_{2}$. So triangle $t_{i}$ is associated to angle $\alpha_{i}=\left\{b, b_{i}\right\}, i=1,2$. Let $a_{i}$ be the common atom of angle $\alpha_{i}, i=1,2$; we always have $a_{1}, a_{2} \subset b$. Tetrahedra (and also wedges) are classified into two disjoint categories. We say $d$ (or $\omega$ ) is a dihedral if $a_{1} \neq a_{2}$ so that $b=a_{1} \cup a_{2}$. We say $d$ (or $\omega$ ) is an improper if $a_{1}=a_{2}$. See Figure 8. This categorization of a tetrahedron as either a dihedral or an improper depends on the details of the Z-system; different Z-systems might cause the same tetrahedron to be categorized in opposite ways. In the example of the Z-system for methanol introduced in Figures 2 and 3, we have the dihedral $\left[H, O, C, H_{1}\right]$ and the impropers $\left[\mathrm{H}_{2}, \mathrm{C}, \mathrm{O}, \mathrm{H}_{1}\right]$ and $\left[\mathrm{H}_{3}, \mathrm{C}, \mathrm{O}, \mathrm{H}_{1}\right]$.

One important consequence of the dichotomy between dihedral and improper is their behavior in relation to orientations. There is a canonical way to assign an orientation to a tetrahedron (3-simplex) which is a dihedral. Let $d$ be a dihedral, where we adopt the notation above, and add the definitions $b_{i}=a_{i} \cup a_{i}^{\prime}, i=$ 1,2 . Then the canonical orientation of the tetrahedron $d$ is $\left[A_{1}^{\prime}, A_{1}, A_{2}, A_{2}^{\prime}\right]$, where $a_{i}=\left\{A_{i}\right\}$ and $a_{i}^{\prime}=\left\{A_{i}^{\prime}\right\}, i=1,2$. We say it is canonical because it does not depend on the ordering of the triangles $t_{1}, t_{2}$ forming the associated wedge, i.e. $\left[A_{1}^{\prime}, A_{1}, A_{2}, A_{2}^{\prime}\right]=\left[A_{2}^{\prime}, A_{2}, A_{1}, A_{1}^{\prime}\right]$ as one can easily check. We will call this the canonical dihedral orientation. Although it is not necessary to do so, it is the usual practice when building a Z-system to assign the canonical dihedral orientation to any tetrahedron which is a dihedral. When a dihedral tetrahedron is equipped with its canonical orientation it is called a torsion. As one can easily check, we have done this for the dihedral in our Z-system for methanol. The situation for impropers is quite different. An orientation needs to be chosen but neither of the two possibilities stands out as a better choice. Unlike dihedrals, the common bond $b$ is oriented, from $a_{1}=a_{2}$ toward the other atom $a_{3}=\left\{A_{3}\right\}$. But since the two triangles are not ordered, no particular orientation is determined. If we add 
information to the tetrahedron $d$ which orders the two triangles as $t_{1}, t_{2}$, then an orientation consistent with this choice is $\left[A_{1}, A_{3}, A_{1}^{\prime}, A_{2}^{\prime}\right]$. But if we reverse the order of the two triangles then we get $\left[A_{1}, A_{3}, A_{2}^{\prime}, A_{1}^{\prime}\right]$, which is clearly the opposite orientation to that obtained from the other ordering. Even though there is no canonical orientation of an improper, a choice of an orientation of an improper is equivalent to a choice of an ordering of the two triangles forming the improper wedge. This explains the arrows on the impropers in Figure 3.

One practical difference between dihedrals and impropers arises from the nature of chemical forces. Since the Z-system $\Gamma^{*}$ determines a coordinate system valid throughout the dense open set $\mathcal{D}_{C}(\Gamma)$, the potential energy of the molecule is a well-defined (except where the energy is infinite due to two non-bonded atoms occupying the same point of space) function on $\mathcal{D}_{P}\left(\Gamma^{*}\right)$. (We are assuming there are no external forces so that the potential energy is invariant under all rigid motions of the molecular configuration.) For biological molecules under most biologically relevant conditions such potential energies are not greatly elevated above their minimum possible values. Thus if the Z-system is chosen correctly we can expect that the bond lengths will be effectively constrained to be within one or two tenths of an angstrom of a minimum energy value. Likewise the bond angles are constrained to be within 0.5 to 1 degrees of a minimum energy value. Improper wedge angles are also constrained to be within 1 or so degrees of a minimum energy value. But dihedral wedge angles are not so strongly constrained under normal biologically important conditions [60]. Thus an interesting mathematical model of a biomolecule is obtained by exactly freezing the values of all the bond lengths, bond angles, and improper wedge angles, but allowing complete freedom for the dihedral wedge angles (unless they are involved in covalently bound rings). However the clarity of this situation can be marred by a poor choice of Z-system, namely if there is an overuse of dihedrals. For example, consider the methyl group in methanol, namely the group of atoms $C, H_{1}, H_{2}, H_{3}$, which is attached to the rest of the molecule via the bond $\{C, O\}$. Under normal biologically relevant conditions the methyl group is well approximated as a rigid body whose primary degree of freedom is its ability to rotate about the axis of the bond $\{C, O\}$. This one degree of freedom should correspond to a single free dihedral. Thus in our Z-system for methanol we use the single dihedral $d_{1}=\left\{H, O, C, H_{1}\right\}$ to describe the orientation of the methyl group relative to the rest of the molecule, and we use the two impropers $\left\{H_{2}, C, O, H_{1}\right\}$ and $\left\{H_{3}, C, O, H_{1}\right\}$ to fix the shape of the methyl group as a rigid body. However it is mathematically possible (but chemically inadvisable) to replace these two impropers with the two dihedrals $d_{2}=\left\{H_{2}, C, O, H\right\}$ and $d_{3}=\left\{H_{3}, C, O, H\right\}$. The rigid body constraint would in this new coordinate system translate into constraints on the differences between pairs of dihedral angles: $\varphi_{d_{2}}-\varphi_{d_{1}}=120^{\circ}$ and $\varphi_{d_{3}}-\varphi_{d_{1}}=$ $-120^{\circ}$. Thus as the methyl group rotates three dihedral angles change, but would do so in concert. Clearly it is preferable to have only one angle change during this rotation. Let $\Gamma_{d}^{3} \subset \Gamma^{3}$ denote the subclass of dihedrals. If the mapping $\Gamma_{d}^{3} \rightarrow \Gamma^{1}$, which takes a dihedral to its common bond, is injective, then the Z-system is sparing in its use of dihedrals. This can always be arranged by converting (if necessary) the extra dihedrals into impropers. Thus, if when constructing a Z-system care is taken not to overuse dihedrals, then a biomolecule (excluding the covalently bonded rings of atoms) can be approximated as a system of linked rigid bodies whose primary independent degrees of flexibility are its torsion angles [33], [24], [72]. 
6.3. Tethering. Chemical reactions give rise to interesting constructions on Zsystems. Suppose molecules described by Z-systems $\Gamma$ and $\Lambda$ approach one another in space and undergo a chemical reaction from which molecules described by Zsystems $\Sigma$ and $\Omega$ emerge. When the reaction is viewed in this way, $\Gamma$ and $\Lambda$ describe the reactants and $\Sigma$ and $\Omega$ describe the products. In order to describe the approach of the reactants toward one another in space and the early stages of the chemical reaction it is necessary to form a Z-system $\Gamma \oplus_{\mu} \Lambda$ for the reactant supermolecule. There are many ways this approach could be described, and a particular choice of how this will be done is represented by the information $\mu$ (see below). Similarly there will be a product supermolecule described by a Z-system $\Sigma \oplus_{\nu} \Omega$. Both of the Z-systems $\Gamma \oplus_{\mu} \Lambda$ and $\Sigma \oplus_{\nu} \Omega$ should be able to describe the shapes of the transition states, i.e. all such shapes should be members of $\mathcal{D}_{C}\left(\Gamma \oplus_{\mu} \Lambda\right) \cap \mathcal{D}_{C}\left(\Sigma \oplus_{\nu} \Omega\right)$. The operation of forming from $\Gamma$ and $\Lambda$ the new Z-system $\Gamma \oplus_{\mu} \Lambda$ using the information $\mu$ will be called tethering. (See Figure 9 for an important example.) We assume that $\Gamma$ is a Z-system on the set $\mathcal{N}$ of $N$ elements, and $\Lambda$ is a Z-system on the set $\mathcal{M}$ of $M$ elements, where $\mathcal{N} \cap \mathcal{M}=\emptyset$. Then $\Gamma \oplus_{\mu} \Lambda$ will be a Z-system on the set $\mathcal{N} \cup \mathcal{M}$ of $N+M$ elements. Thus tethering conserves atoms. One simple way to specify the tethering information $\mu$ is to give two sites, $\gamma$ from $\Gamma$ and $\lambda$ from $\Lambda$, i.e. $\mu=\{\gamma, \lambda\}$. Thus we must specify a triple $\gamma=\left(i_{0}, i_{1}, i_{2}\right)$ of elements of $\mathcal{N}$ such that $\left\{i_{0}\right\} \in \Gamma^{0}$ (necessarily true), $\left\{i_{0}, i_{1}\right\} \in \Gamma^{1}$, and $\left\{i_{0}, i_{1}, i_{2}\right\} \in \Gamma^{2}$; this defines a site from $\Gamma$. Likewise we must specify a triple $\lambda=\left(j_{0}, j_{1}, j_{2}\right)$ of elements of $\mathcal{M}$ such that $\left\{j_{0}\right\} \in \Lambda^{0}$ (necessarily true), $\left\{j_{0}, j_{1}\right\} \in \Lambda^{1}$, and $\left\{j_{0}, j_{1}, j_{2}\right\} \in \Lambda^{2}$. Clearly we will have $\left(\Gamma \oplus_{\mu} \Lambda\right)^{0}=\Gamma^{0} \cup \Lambda^{0}$. We define

$$
\begin{aligned}
& \left(\Gamma \oplus_{\mu} \Lambda\right)^{1}=\Gamma^{1} \cup \Lambda^{1} \cup\left\{\left\{i_{0}, j_{0}\right\}\right\} \\
& \left(\Gamma \oplus_{\mu} \Lambda\right)^{2}=\Gamma^{2} \cup \Lambda^{2} \cup\left\{\left\{i_{0}, i_{1}, j_{0}\right\},\left\{j_{0}, j_{1}, i_{0}\right\}\right\} \\
& \left(\Gamma \oplus_{\mu} \Lambda\right)^{3}=\Gamma^{3} \cup \Lambda^{3} \cup\left\{\left\{i_{0}, i_{1}, i_{2}, j_{0}\right\},\left\{i_{0}, i_{1}, j_{0}, j_{1}\right\},\left\{j_{0}, j_{1}, j_{2}, i_{0}\right\}\right\} \\
& \left(\Gamma \oplus_{\mu} \Lambda\right)_{*}^{3}=\Gamma_{*}^{3} \cup \Lambda_{*}^{3} \cup\left\{\left[j_{0}, i_{0}, i_{1}, i_{2}\right],\left[i_{1}, i_{0}, j_{0}, j_{1}\right],\left[i_{0}, j_{0}, j_{1}, j_{2}\right]\right\} .
\end{aligned}
$$

The new bond $\left\{i_{0}, j_{0}\right\}$ is called the tether. In regard to orientations we note that the central tetrahedron must be a dihedral, so we choose its orientation to be canonical, i.e. $\left[i_{1}, i_{0}, j_{0}, j_{1}\right]$. The other two tetrahedra could be either dihedrals or impropers, but we assign their orientations as follows: $\left[j_{0}, i_{0}, i_{1}, i_{2}\right]$ and $\left[i_{0}, j_{0}, j_{1}, j_{2}\right]$. In the dihedral case, this is the canonical orientation. But in the improper case we have effectively decided that the new triangle should be rotated into the old triangle.

Lemma. $\Gamma \oplus_{\mu} \Lambda$ is a well-defined Z-system.

Proof. Conditions (3) and (4) in the definition of Z-system are verified by inspection. Since $\left|\Gamma^{1}\right|=N-1$ and $\left|\Lambda^{1}\right|=M-1$ we have that $\left|\left(\Gamma \oplus_{\mu} \Lambda\right)^{1}\right|=$ $(N-1)+(M-1)+1=N+M-1$. Since the graph $\left(\left(\Gamma \oplus_{\mu} \Lambda\right)^{0},\left(\Gamma \oplus_{\mu} \Lambda\right)^{1}\right)$ is obviously connected, it must be a tree. By similar counting arguments we see that both $\left(\left(\Gamma \oplus_{\mu} \Lambda\right)^{1},\left(\Gamma \oplus_{\mu} \Lambda\right)^{2}\right)$ and $\left(\left(\Gamma \oplus_{\mu} \Lambda\right)^{2},\left(\Gamma \oplus_{\mu} \Lambda\right)^{3}\right)$ are trees. Hence condition (5) also holds true.

It should be clear that $\Gamma \oplus_{\mu} \Lambda=\Lambda \oplus_{\mu} \Gamma$. Furthermore if $\gamma$ is a site in $\Gamma, \lambda_{1}, \lambda_{2}$ are sites in $\Lambda$, and $\delta$ is a site in another Z-system $\Delta$, then we have an associative property:

$$
\Gamma \oplus_{\left\{\gamma, \lambda_{1}\right\}}\left(\Lambda \oplus_{\left\{\lambda_{2}, \delta\right\}} \Delta\right)=\left(\Gamma \oplus_{\left\{\gamma, \lambda_{1}\right\}} \Lambda\right) \oplus_{\left\{\lambda_{2}, \delta\right\}} \Delta
$$



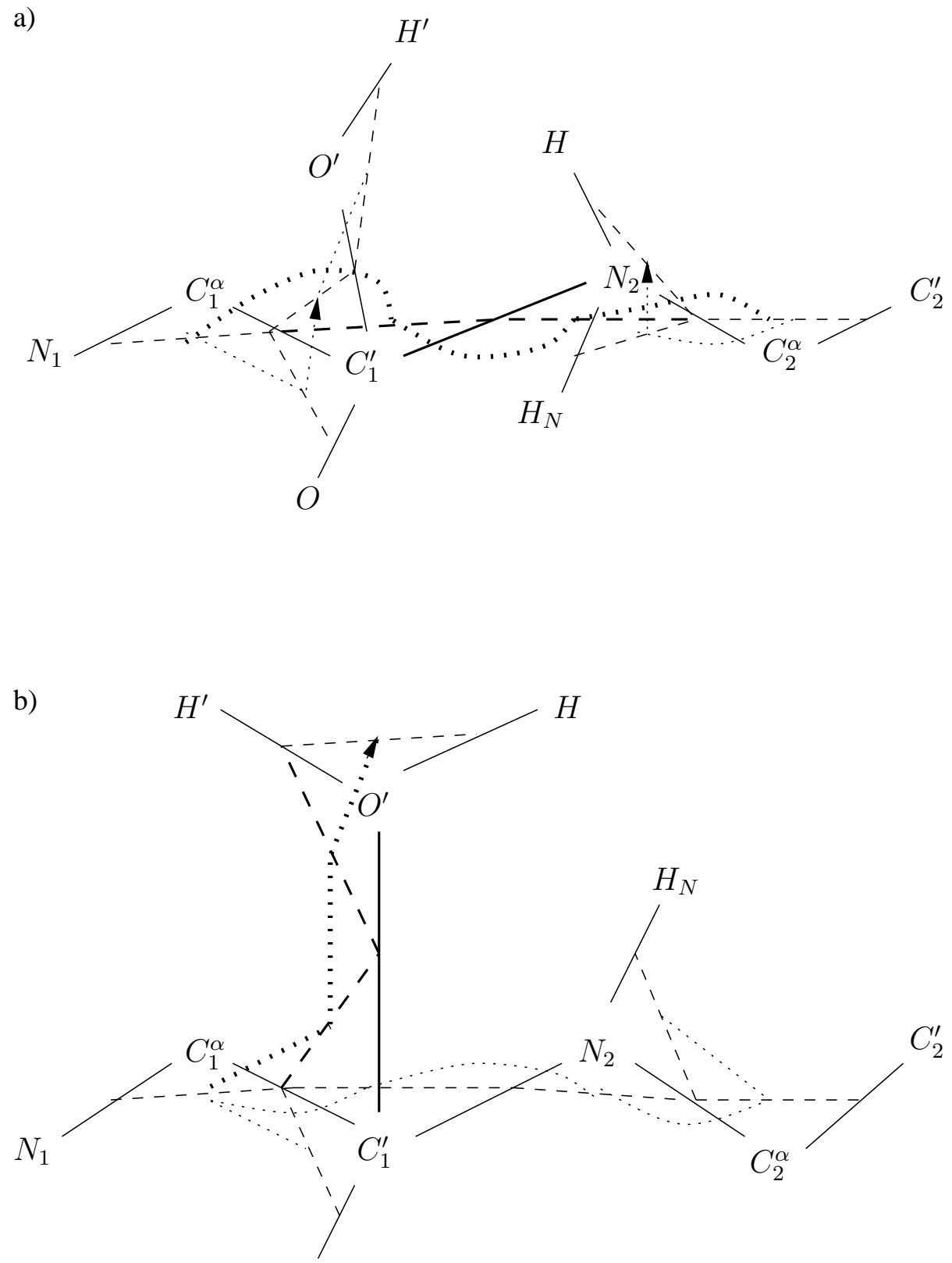

$O$

Figure 9. a) A tethered Z-system for the reactant supermolecule: two amino acids approach one another. b) A tethered Z-system for the product supermolecule: a water molecule moves away from a dipeptide. This example is illustrative; see [47] and [21] for more realistic reaction mechanisms. The tethers (bonds) are shown as dark lines, the tether angles as dark dashed lines, and the tether wedges are shown as dark dotted curves or lines. 
Tethering can also be done with labeled Z-systems, where the numerical labels of the six added simplices specify the relative position and orientation of the two molecules. This is an interesting symmetrical way to do this.

Figure 9 shows that the tethering process can produce Z-systems which overuse dihedrals. The set of wedges can be changed to remove this problem and the new wedges can be assigned labels according to the theory in section 3.4 so that the shape is not changed.

6.4. Gluing. Z-systems for large biomolecules are extremely laborious to generate from scratch. As compact as a Z-matrix is, it nevertheless has one row for each atom, and biomolecules can easily have thousands of atoms. The details of the structure of these molecules cannot be neglected if a mechanistic understanding of biological processes is to be achieved so we must find ways to deal with the huge quantity of information in large Z-systems. One idea is to build up large Z-systems from smaller Z-systems by gluing the smaller pieces together. Biomolecules lend themselves to this approach because they are polymers, synthesized from many copies of smaller molecules called monomers. But as we have seen, mimicking the chemical reactions involved in joining these monomers together is rather complicated in its detail. It is desirable to have a gluing operation which can bypass the chemical processes and go straight to the final result. In fact, when one builds plastic models of molecules one uses exactly such a gluing operation. It is interesting that Z-systems are perfectly suited to such an operation.

Suppose Z-systems $\Gamma$ and $\Lambda$ on disjoint sets $\mathcal{N}$ and $\mathcal{M}$ respectively, and with sites $\gamma=\left(i_{0}, i_{1}, i_{2}\right) \in \operatorname{vert} \mathcal{S}(\Gamma)$ and $\lambda=\left(j_{0}, j_{1}, j_{2}\right) \in$ vert $\mathcal{S}(\Lambda)$ are given. We require that $\left\{i_{0}\right\}$ is a leaf vertex in the tree $\left(\Gamma^{0}, \Gamma^{1}\right)$, and that $\left\{j_{0}\right\}$ is a leaf vertex in the tree $\left(\Lambda^{0}, \Lambda^{1}\right)$. We intend to define a new Z-system $\Gamma *_{\mu} \Lambda$, where $\mu=\{\gamma, \lambda\}$. It will be a Z-system on the set $\mathcal{L}=\left(\mathcal{N} \backslash\left\{i_{0}\right\}\right) \cup\left(\mathcal{M} \backslash\left\{j_{0}\right\}\right)$, which (together with the mappings $\left.\iota_{\mathcal{N}}, \iota_{\mathcal{M}}\right)$ is the pushout of the following diagram:

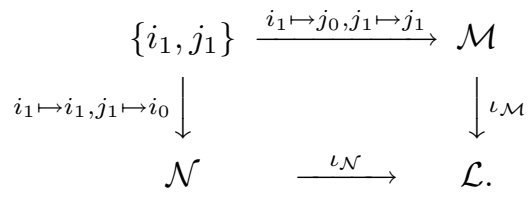

Given any set $S$ and any mappings $f: \mathcal{M} \rightarrow S, g: \mathcal{N} \rightarrow S$ such that $f\left(j_{0}\right)=g\left(i_{1}\right)$ and $f\left(j_{1}\right)=g\left(i_{0}\right)$ there exists a unique mapping $h: \mathcal{L} \rightarrow S$ such that $f=h \circ \iota_{\mathcal{M}}$ and $g=h \circ \iota_{\mathcal{N}}$. This pushout property requires the definitions:

$$
\iota_{\mathcal{N}}(i)=\left\{\begin{array}{ll}
i & i \in \mathcal{N} \backslash\left\{i_{0}\right\} \\
j_{1} & i=i_{0}
\end{array}, \quad \iota_{\mathcal{M}}(j)=\left\{\begin{array}{ll}
j & j \in \mathcal{M} \backslash\left\{j_{0}\right\} \\
i_{1} & j=j_{0}
\end{array},\right.\right.
$$

and hence $h(l)=\left\{\begin{array}{ll}f(l) & l \in \mathcal{M} \backslash\left\{j_{0}\right\} \\ g(l) & l \in \mathcal{N} \backslash\left\{i_{0}\right\}\end{array}\right.$. (We describe these mappings in such an elaborate manner to suggest how the construction might be generalized to the case $n \neq 3$, where instead of gluing along a 1 simplex, one glues along an $n-2$ simplex. The upper horizontal and left vertical mappings of the pushout rectangle would be required to be monomorphisms of Z-systems.) Now we define

$$
\begin{aligned}
& \left(\Gamma *_{\mu} \Lambda\right)^{1}=\left\{\iota_{\mathcal{N}}(b) \mid b \in \Gamma^{1}\right\} \cup\left\{\iota_{\mathcal{M}}(b) \mid b \in \Lambda^{1}\right\}, \\
& \left(\Gamma *_{\mu} \Lambda\right)^{2}=\left\{\iota_{\mathcal{N}}(t) \mid t \in \Gamma^{2}\right\} \cup\left\{\iota_{\mathcal{M}}(t) \mid t \in \Lambda^{2}\right\},
\end{aligned}
$$




$$
\left(\Gamma *_{\mu} \Lambda\right)_{*}^{3}=\left\{\iota_{\mathcal{N}}\left(\tau^{*}\right) \mid \tau^{*} \in \Gamma_{*}^{3}\right\} \cup\left\{\iota_{\mathcal{M}}\left(\tau^{*}\right) \mid \tau^{*} \in \Lambda_{*}^{3}\right\} \cup\left\{\left[i_{2}, i_{1}, j_{1}, j_{2}\right]\right\} .
$$

The mappings $\iota_{\mathcal{N}}$ and $\iota_{\mathcal{M}}$ take oriented 3 simplices into oriented 3 simplices in the obvious manner. The new 3 simplex $\left\{i_{2}, i_{1}, j_{1}, j_{2}\right\}$ is a dihedral, and it is given the canonical orientation $\left[i_{2}, i_{1}, j_{1}, j_{2}\right]$.

Lemma. The above definitions define a Z-system $\Gamma *_{\mu} \Lambda$ on $\mathcal{L}$.

Proof. Since $\left\{i_{0}\right\}$ is a leaf vertex in the tree $\left(\Gamma^{0}, \Gamma^{1}\right)$ the bond $\left\{i_{0}, i_{1}\right\}$ is the only bond in $\Gamma^{1}$ incident on $\left\{i_{0}\right\}$. So if $b \in \Gamma^{1} \backslash\left\{\left\{i_{0}, i_{1}\right\}\right\}$ then $\iota_{\mathcal{N}}(b)=b$. Likewise since $\left\{j_{0}\right\}$ is a leaf vertex in the tree $\left(\Lambda^{0}, \Lambda^{1}\right)$ the bond $\left\{j_{0}, j_{1}\right\}$ is the only bond in $\Lambda^{1}$ incident on $\left\{j_{0}\right\}$. So if $b \in \Lambda^{1} \backslash\left\{\left\{j_{0}, j_{1}\right\}\right\}$ then $\iota_{\mathcal{M}}(b)=b$. Clearly $\iota_{\mathcal{N}}\left(\left\{i_{0}, i_{1}\right\}\right)=$ $\iota_{\mathcal{M}}\left(\left\{j_{0}, j_{1}\right\}\right)=\left\{i_{1}, j_{1}\right\}$. Thus

$$
\left(\Gamma *_{\mu} \Lambda\right)^{1}=\left(\Gamma^{1} \backslash\left\{\left\{i_{0}, i_{1}\right\}\right\}\right) \cup\left(\Lambda^{1} \backslash\left\{\left\{j_{0}, j_{1}\right\}\right\}\right) \cup\left\{\left\{i_{1}, j_{1}\right\}\right\} .
$$

It is easy to see that $\left(\left(\Gamma *_{\mu} \Lambda\right)^{0},\left(\Gamma *_{\mu} \Lambda\right)^{1}\right)$ is a connected graph. Since $\left(\Gamma *_{\mu} \Lambda\right)^{0}=\left(\begin{array}{l}\mathcal{L} \\ 1\end{array}\right)$ has $(N-1)+(M-1)=N+M-2$ elements, and $\left(\Gamma *{ }_{\mu} \Lambda\right)^{1}$ has $(N-2)+(M-2)+1=$ $N+M-3$ elements, this graph must be a tree.

To see that condition (3) is true for $k=2$, let $t \in\left(\Gamma *_{\mu} \Lambda\right)^{2}$ and consider the set $\tilde{\sigma}(t)=\left\{b \in\left(\Gamma *_{\mu} \Lambda\right)^{1} \mid b \subset t\right\}$. We must show that $\tilde{\sigma}(t)$ has exactly two elements. We may assume that $t=\iota_{\mathcal{N}}\left(t^{\prime}\right)$ for $t^{\prime} \in \Gamma^{2}$, since the argument for $t^{\prime} \in \Lambda^{2}$ is completely analogous. Suppose first of all that $i_{0} \notin t^{\prime}$. Thus $t^{\prime} \subset \mathcal{N} \backslash\left\{i_{0}\right\}$. Therefore $t^{\prime}=t$. There exist exactly two bonds $b_{1}, b_{2} \in \Gamma^{1}$ such that $b_{1} \subset t$ and $b_{2} \subset t$. Therefore $b_{1}, b_{2} \subset \mathcal{N} \backslash\left\{i_{0}\right\}$ and hence $b_{1}, b_{2} \in\left(\Gamma^{1} \backslash\left\{\left\{i_{0}, i_{1}\right\}\right\}\right) \subset\left(\Gamma *_{\mu} \Lambda\right)^{1}$. Thus $\left\{b_{1}, b_{2}\right\} \subset \tilde{\sigma}(t)$. Now suppose $b \in \tilde{\sigma}(t)$, i.e. $b \in\left(\Gamma *_{\mu} \Lambda\right)^{1}$ and $b \subset t$. Since $b \subset \mathcal{N} \backslash\left\{\left\{i_{0}\right\}\right\}$ we have $b \in \Gamma^{1} \backslash\left\{\left\{i_{0}, i_{1}\right\}\right\}$. Thus $b \in\left\{b_{1}, b_{2}\right\}$ and $\tilde{\sigma}(t)=\left\{b_{1}, b_{2}\right\}$, as desired. On the other hand if $i_{0} \in t^{\prime}$ then $b_{1}=\left\{i_{0}, i_{1}\right\}$ (being the only bond in $\Gamma^{1}$ incident on $\left.\left\{i_{0}\right\}\right)$ satisfies $b_{1} \subset t^{\prime}$. There is exactly one other bond $b_{2} \in \Gamma^{1}$ such that $b_{2} \subset t^{\prime}$, and we must have $i_{0} \notin b_{2}$. Thus $b_{2}=\left\{i_{1}, i\right\}$, where $i \in \mathcal{N} \backslash\left\{i_{0}, i_{1}\right\}$. Therefore $\iota_{\mathcal{N}}\left(b_{1}\right)=\left\{j_{1}, i_{1}\right\}$ and $\iota_{\mathcal{N}}\left(b_{2}\right)=b_{2}$ are two members of $\tilde{\sigma}(t) .\left\{j_{1}, i\right\} \notin \tilde{\sigma}(t)$ since $\left\{i_{0}, i\right\} \notin \Gamma^{1}$. Hence $\tilde{\sigma}(t)=\left\{\left\{j_{1}, i_{1}\right\},\left\{i_{1}, i\right\}\right\}$, as desired. The sets $\left\{\iota_{\mathcal{M}}(t) \mid t \in \Gamma^{2}\right\}$ and $\left\{\iota_{\mathcal{N}}(t) \mid t \in \Lambda^{2}\right\}$ are disjoint, hence $\left(\Gamma *_{\mu} \Lambda\right)^{2}$ has $(N-2)+(M-2)=N+M-4$ elements. The graph $\left(\left(\Gamma *_{\mu} \Lambda\right)^{1},\left(\Gamma *_{\mu} \Lambda\right)^{2}\right)$ is clearly connected, so it must be a tree. Thus condition (5), $k=2$ is verified.

Again we must show that condition (3) with $k=3$ is true, so let $d \in\left(\Gamma *_{\mu} \Lambda\right)^{3}$ and consider the set $\tilde{\sigma}(d)=\left\{t \in\left(\Gamma *_{\mu} \Lambda\right)^{2} \mid t \subset d\right\}$. We must show this set has exactly two elements. First suppose $d=\left\{i_{2}, i_{1}, j_{1}, j_{2}\right\}$. Clearly $\left\{t, t^{\prime}\right\} \subset \tilde{\sigma}(d)$ where $t=\left\{i_{2}, i_{1}, j_{1}\right\}=\iota_{\mathcal{N}}\left(\left\{i_{0}, i_{1}, i_{2}\right\}\right)$ and $t^{\prime}=\left\{i_{1}, j_{1}, j_{2}\right\}=\iota_{\mathcal{M}}\left(\left\{j_{0}, j_{1}, j_{2}\right\}\right)$. If $t^{\prime \prime} \in \tilde{\sigma}(d)$ then either $t^{\prime \prime}=\iota_{\mathcal{N}}(\tilde{t})$ for $\tilde{t} \in \Gamma^{2}$ or $t^{\prime \prime}=\iota_{\mathcal{M}}(\tilde{t})$ for $\tilde{t} \in \Lambda^{2}$. Thus either $\tilde{t}=\left\{i_{0}, i_{1}, i_{2}\right\}$ or $\tilde{t}=\left\{j_{0}, j_{1}, j_{2}\right\}$, implying that $t^{\prime \prime} \in\left\{t, t^{\prime}\right\}$, and hence $\tilde{\sigma}(d)=\left\{t, t^{\prime}\right\}$ as desired. Now suppose that $d=\iota_{\mathcal{N}}\left(d^{\prime}\right)$ where $d^{\prime} \in \Gamma^{3}$. (The case where $d=\iota_{\mathcal{M}}\left(d^{\prime}\right)$ where $d^{\prime} \in \Lambda^{3}$ is argued analogously.) Let $\tilde{\sigma}\left(d^{\prime}\right)=\left\{t, t^{\prime}\right\}$. Then $\left\{\iota_{\mathcal{N}}(t), \iota_{\mathcal{N}}\left(t^{\prime}\right)\right\} \subset \tilde{\sigma}(d)$. On the other hand if $t^{\prime \prime} \in\left(\Gamma *_{\mu} \Lambda\right)^{2}$ and $t^{\prime \prime} \subset d$ then we must have $t^{\prime \prime}=\iota_{\mathcal{N}}(\tilde{t})$ for $\tilde{t} \in \Gamma^{2}$. Since $\iota_{\mathcal{N}}$ is an injection and $\iota_{\mathcal{N}}(\tilde{t}) \subset \iota_{\mathcal{N}}\left(d^{\prime}\right)$, it follows that $\tilde{t} \subset d^{\prime}$, and hence $\tilde{t} \in\left\{t, t^{\prime}\right\}$. Thus $\tilde{\sigma}(d)=\left\{\iota_{\mathcal{N}}(t), \iota_{\mathcal{N}}\left(t^{\prime}\right)\right\}$, as desired. In both cases $d$ is the union of the two triangles in $\tilde{\sigma}(d)$, so $d$ is uniquely determined by $\tilde{\sigma}(d)$, finishing the proof of condition (3) with $k=3$. The union defining $\left(\Gamma *_{\mu} \Lambda\right)^{3}$ is a disjoint union, so $\left(\Gamma *_{\mu} \Lambda\right)^{3}$ has $(N-3)+(M-3)+1=N+M-5$ elements. The tetrahedron $\left\{i_{2}, i_{1}, j_{1}, j_{2}\right\}$ connects the triangle $\iota_{\mathcal{N}}\left(\left\{i_{0}, i_{1}, i_{2}\right\}\right)=\left\{i_{2}, i_{1}, j_{1}\right\}$ to the triangle $\iota_{\mathcal{M}}\left(\left\{j_{0}, j_{1}, j_{2}\right\}\right)=\left\{i_{1}, j_{1}, j_{2}\right\}$, so the graph $\left(\left(\Gamma *_{\mu} \Lambda\right)^{2},\left(\Gamma *_{\mu} \Lambda\right)^{3}\right)$ is 


$\begin{array}{cccccccc}C & & & & & & \\ H_{*} & C & & & & O & & \\ H_{1} & C & H_{*} & & & H & O & \\ H_{2} & C & H_{*} & H_{1} & & H^{*} & O & H \\ H_{3} & C & H_{*} & H_{1} & & & & \end{array}$

FiguRE 10. Unlabeled Z-matrices for methane and water.

connected and hence a tree. Condition (5) with $k=3$ in the definition of Z-system is now verified.

Since $\left\{i_{2}, i_{1}, j_{1}\right\} \cup\left\{i_{1}, j_{1}, j_{2}\right\}=\left\{i_{2}, i_{1}, j_{1}, j_{2}\right\}$ and $\left\{i_{2}, i_{1}, j_{1}\right\} \cap\left\{i_{1}, j_{1}, j_{2}\right\}=$ $\left\{i_{1}, j_{1}\right\} \in\left(\Gamma *_{\mu} \Lambda\right)^{1}$, we see the intersection property (condition (4)) is satisfied in this case. Elsewhere it is a consequence of the intersection property in each of the Z-systems $\Gamma$ and $\Lambda$. So $\Gamma *_{\mu} \Lambda$ is an unoriented Z-system.

Gluing is also commutative and associative under the same assumptions (distinct sites) as for tethering together with the always necessary leaf assumptions.

This gluing operation on Z-systems can be illustrated by the example of combining methane and water to make methanol. Let unlabeled Z-matrices determining Z-systems $\Gamma$ and $\Lambda$ for methane and water respectively be given in Figure 10 . As discussed in section 5.2 we use a Z-matrix to define a Z-system, where we ignore the root site and the leaf-picking order. We choose the site $\gamma=\left(H_{*}, C, H_{1}\right)$ in methane, and the site $\lambda=\left(H^{*}, O, H\right)$ in water. We have $\mathcal{N}=\left\{C, H_{*}, H_{1}, H_{2}, H_{3}\right\}$ and $\mathcal{M}=\left\{O, H, H^{*}\right\}$ and the mappings

$$
\begin{aligned}
& \begin{array}{c|cccccccc}
A & C & H_{*} & H_{1} & H_{2} & H_{3} & O & H & H^{*} \\
--- & -- & -- & -- & -- & -- & -- & -- & --
\end{array} \\
& \begin{array}{l|llllllll}
\iota_{\mathcal{N}}(A) & C & O & H_{1} & H_{2} & H_{3} & & & \\
\iota_{\mathcal{M}}(A) & & & & & & O & H & C
\end{array}
\end{aligned}
$$

The result of gluing is the Z-system $\Gamma *_{\mu} \Lambda$ on the set $\mathcal{L}=\left\{C, H_{1}, H_{2}, H_{3}, O, H\right\}$, where

$$
\begin{aligned}
& \left(\Gamma *_{\mu} \Lambda\right)^{1}=\left\{\left\{C, H_{1}\right\},\left\{C, H_{2}\right\},\left\{C, H_{3}\right\},\{O, H\},\{C, O\}\right\} \\
& \left(\Gamma *_{\mu} \Lambda\right)^{2}=\left\{\left\{H_{1}, C, O\right\},\left\{H_{2}, C, O\right\},\left\{H_{3}, C, O\right\},\{C, O, H\}\right\} \\
& \left(\Gamma *_{\mu} \Lambda\right)_{*}^{3}=\left\{\left[H_{2}, C, O, H_{1}\right],\left[H_{3}, C, O, H_{1}\right],\left[H_{1}, C, O, H\right]\right\} .
\end{aligned}
$$

This Z-system for methanol coincides with the one pictured in Figures 2 and 3 . As usual there are many Z-matrices one could write for this Z-system, such as the one given in section 5 , but none of them are obtained from the two initial Z-matrices by a simple manipulation, even if we could easily reorder Z-matrices [81].

Labeled Z-systems can be glued as above to yield another labeled Z-system. The gluing information $\mu$, in addition to the two sites $\gamma$ and $\lambda$, must contain a bond length for the bond $\left\{i_{1}, j_{1}\right\}$ and a unit modulus complex number as the wedge angle coordinate for the oriented 3 simplex $\left[i_{2}, i_{1}, j_{1}, j_{2}\right]$. The other labels are carried over from the corresponding simplices of $\Gamma$ or $\Lambda$. The bond length of $\left\{i_{1}, j_{1}\right\}$ is often determined by the element types of $i_{1}$ and $j_{1}$, since $\left\{i_{1}, j_{1}\right\}$ is usually a single bond. It is possible to glue "building block" versions of amino acid Z-systems so that the new bond $\left\{i_{1}, j_{1}\right\}$ is the peptide bond, which has a wellknown bond length. The shape of the substituent atoms to $\left\{i_{1}\right\}$ and $\left\{j_{1}\right\}$ can be 
chosen so that they are correct after gluing; since the peptide bond has a partial double bond character these shapes will not be the correct ones for individual amino acids in solution - hence the name "building block". The (dihedral) wedge angle coordinate is often not uniquely determined at normal biological conditions. However, when gluing amino acid building blocks, this angle (called $\omega$ ) is usually close to $\pi$ (leading to a "trans" peptide unit). Thus labeled Z-systems provide the fundamental mathematical tool we need to begin a systematic study of biomolecular geometry.

6.5. Five-Membered Rings. The most serious difficulty in using Z-systems in the study of biomolecular geometry is the presence of flexible covalently bound rings [79], [18], [19], [61], [26]. Flexible five-membered rings are present in both proteins (i.e. proline) and in all nucleic acids (the furanose ring) [3], [55], [57], [73], [1]. Six-membered rings are in sugars (see section 6.7). Larger flexible rings are frequently present (via disulfide bonds) in proteins, and constitute an important constraint on their flexibility [46].

Consider a five-membered ring. If $\mathcal{N}=\{0,1,2,3,4\}$ then the orbit space $G_{a} \| \mathcal{B}$ is $15-6=9$ dimensional. Because of the five covalent bonds of the ring, it is desirable to use those bond lengths as five of the nine coordinates. This clearly takes us outside the realm of Z-systems. A simple possibility (see [66]) is to use as additional coordinates the bond angles $\{\{0,1\},\{1,2\}\}$ and $\{\{1,2\},\{2,3\}\}$ and the dihedral wedge angles $[0,1,2,3]$ and $[1,2,3,4]$. The cosine of the angle $\{\{2,3\},\{3,4\}\}$ is found by solving a quadratic equation derived by imposing the distance constraint between atoms $\{0\}$ and $\{4\}$. There are obviously generically either two or zero real solutions, so these nine coordinates either do not determine any shape or do not determine a unique shape. If we always choose the larger of the two solutions of the quadratic equation, the coordinate domain (which cannot be dense) and the parameter domain still need to be characterized. Furthermore, practically speaking, the measure of the angle $\{\{2,3\},\{3,4\}\}$ is usually known with greater certainty than that of the wedge angle $[1,2,3,4]$.

Another more complex (yet more symmetrical) choice of the four coordinates supplementing the five bond lengths has been proposed and studied [65]. This coordinate system has the benefit that the primary degree of flexibility of the ring is described by one of the coordinates, the so-called pseudorotation phase angle. However, the coordinate and parameter domains for this system have not been characterized.

An interesting alternative to these approaches can be based directly on Z-system theory. Consider the Z-system $\Gamma$ on $\mathcal{N}$ derived from the following Z-matrix (see also Figure 11).

$\begin{array}{llll}1 & & & \\ 2 & 1 & & \\ 0 & 2 & 1 & \\ 3 & 0 & 2 & 1 \\ 4 & 3 & 0 & 2\end{array}$

This system has the "bonds" $\{0,2\}$ and $\{3,0\}$ which are not covalently bonded pairs of atoms. Nevertheless our main theorem gives us a diffeomorphism $\hat{\eta}: \mathcal{D}_{C}(\Gamma) \rightarrow$ $\mathcal{D}_{P}\left(\Gamma^{*}\right)$. As usual $\mathcal{D}_{C}(\Gamma)$ is the set of all shapes $\mathcal{O}$ for which the three triangles $\{0,2,1\},\{3,0,2\},\{4,3,0\}$ represent non collinear spatial triangles. Also $\mathcal{D}_{P}\left(\Gamma^{*}\right)=$ 


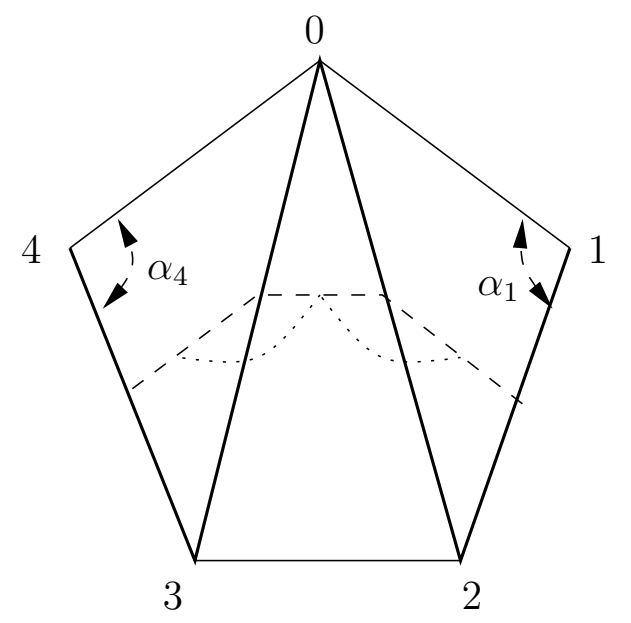

Figure 11. A Z-system $\Gamma$ for a Five-Membered Ring.

$(0, \infty)^{\Gamma^{1}} \times(-1,1)^{\Gamma^{2}} \times\left(S^{1}\right)^{\Gamma_{*}^{3}}$. Define

$$
\begin{aligned}
& \Lambda^{1}=\{\{0,1\},\{1,2\},\{2,3\},\{3,4\},\{4,0\}\}, \\
& \Lambda^{2}=\left\{\alpha_{1}=\{\{0,1\},\{1,2\}\}, \alpha_{4}=\{\{3,4\},\{4,0\}\}\right\} .
\end{aligned}
$$

Define the mapping $\xi^{\prime}:(0, \infty)^{\Gamma^{1}} \times(-1,1)^{\Gamma^{2}} \rightarrow(0, \infty)^{\Lambda^{1}} \times(-1,1)^{\Lambda^{2}}$ by the rule $\xi^{\prime}\left(L^{\prime}, C^{\prime}\right)=(L, C)$, where

$$
\begin{aligned}
L_{\{0,1\}} & =\sqrt{\left(L_{\{1,2\}}^{\prime}\right)^{2}+\left(L_{\{0,2\}}^{\prime}\right)^{2}-2 L_{\{1,2\}}^{\prime} L_{\{0,2\}}^{\prime} C_{\{0,2,1\}}^{\prime}} \\
L_{\{1,2\}} & =L_{\{1,2\}}^{\prime} \\
L_{\{2,3\}} & =\sqrt{\left(L_{\{0,2\}}^{\prime}\right)^{2}+\left(L_{\{0,3\}}^{\prime}\right)^{2}-2 L_{\{0,2\}}^{\prime} L_{\{0,3\}}^{\prime} C_{\{3,0,2\}}^{\prime}} \\
L_{\{3,4\}} & =L_{\{3,4\}}^{\prime} \\
L_{\{4,0\}} & =\sqrt{\left(L_{\{0,3\}}^{\prime}\right)^{2}+\left(L_{\{4,3\}}^{\prime}\right)^{2}-2 L_{\{0,3\}}^{\prime} L_{\{4,3\}}^{\prime} C_{\{4,3,0\}}^{\prime}} \\
C_{\alpha_{1}} & =\frac{L_{\{1,2\}}^{\prime}-L_{\{0,2\}}^{\prime} C_{\{0,2,1\}}^{\prime}}{L_{\{0,1\}}} \\
C_{\alpha_{4}} & =\frac{L_{\{4,3\}}^{\prime}-L_{\{0,3\}}^{\prime} C_{\{4,3,0\}}^{\prime}}{L_{\{4,0\}}}
\end{aligned}
$$

This mapping simply recoordinatizes each of the three triangles in $\Gamma^{2}$. Define $\Delta \subset(0, \infty)^{\Lambda^{1}} \times(-1,1)^{\Lambda^{2}}$ to be the range of $\xi^{\prime}$. The new coordinatization for the triangle $\{0,2,3\}$ is in terms of the lengths of its three sides, and this entails a restriction on the possible triples of lengths. Hence

$$
\begin{aligned}
\Delta=\{(L, C) \mid & L: \Lambda^{1} \rightarrow(0, \infty), C: \Lambda^{2} \rightarrow(-1,1), \text { such that } \\
& {\left[\left(L_{\{0,2\}}^{\prime}\right)^{2}+\left(L_{\{0,3\}}^{\prime}\right)^{2}-\left(L_{\{2,3\}}\right)^{2}\right]^{2}<4\left(L_{\{0,2\}}^{\prime}\right)^{2}\left(L_{\{0,3\}}^{\prime}\right)^{2}, } \\
& \text { where }\left(L_{\{0,2\}}^{\prime}\right)^{2}=\left(L_{\{0,1\}}\right)^{2}+\left(L_{\{1,2\}}\right)^{2}-2 L_{\{0,1\}} L_{\{1,2\}} C_{\alpha_{1}}, \\
& \text { and } \left.\left(L_{\{0,3\}}^{\prime}\right)^{2}=\left(L_{\{0,4\}}\right)^{2}+\left(L_{\{4,3\}}\right)^{2}-2 L_{\{0,4\}} L_{\{4,3\}} C_{\alpha_{4}}\right\} .
\end{aligned}
$$




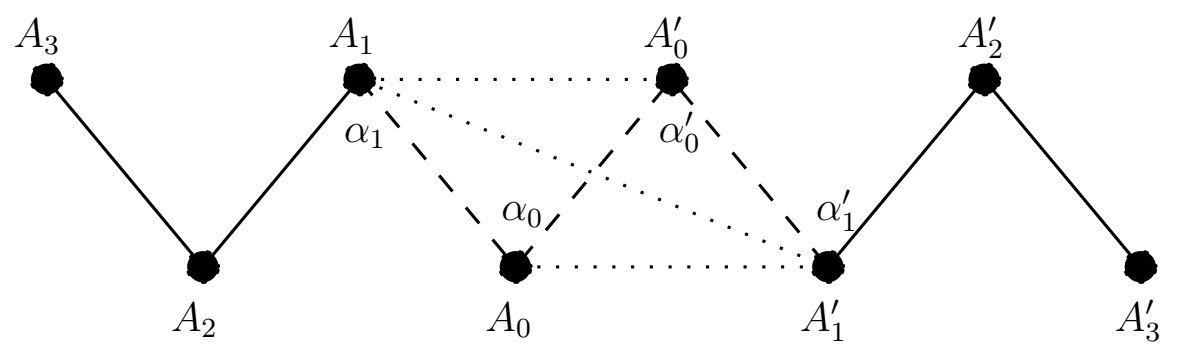

Figure 12. Bridging between sites $r=\left(A_{1}, A_{2}, A_{3}\right)$ and $r^{\prime}=$ $\left(A_{1}^{\prime}, A_{2}^{\prime}, A_{3}^{\prime}\right)$. Atoms $\left\{A_{0}\right\}$ and $\left\{A_{0}^{\prime}\right\}$ are added with distance constraints shown as darker dashed lines. Constrained angles $\alpha_{1}, \alpha_{0}, \alpha_{0}^{\prime}, \alpha_{1}^{\prime}$ are also shown.

If $\xi$ is equal to $\xi^{\prime}$ with its codomain restricted to $\Delta$ then $\xi$ is a diffeomorphism (as the reader is invited to check by explicitly constructing its inverse mapping). If $\mathcal{D}_{P}(\Lambda)=\Delta \times\left(S^{1}\right)^{\Gamma_{*}^{3}}$, then $(\xi \times 1) \circ \hat{\eta}$ is a diffeomorphism between $\mathcal{D}_{C}(\Gamma)$ and $\mathcal{D}_{P}(\Lambda)$. This is an internal coordinate system for the five-membered ring where both the coordinate and the parameter domains are explicitly identified. The coordinates are the five bond lengths $L_{\{0,1\}}, L_{\{1,2\}}, L_{\{2,3\}}, L_{\{3,4\}}, L_{\{4,0\}}$, the two bond-angle cosines $C_{\alpha_{1}}, C_{\alpha_{4}}$, and the two wedge angles $\varphi_{[3,0,2,1]}, \varphi_{[4,3,0,2]}$. The two wedge angle coordinates are called flap angle coordinates in this case. Pseudorotation cycles form closed paths in the flap angle plane and in the bond angle plane. Flap angle coordinates have also appeared in the study of rings with more than five atoms [48], [39].

This result on five-membered rings suggests that there may be interesting generalizations of our coordinatization theorem (section 4.1) where $\Lambda$ is more general than a Z-system, $\mathcal{D}_{C}(\Lambda)$ has the usual definition, and where $\mathcal{D}_{P}(\Lambda)$ is a semi-algebraic set [12], [88].

6.6. Bridging Algorithm, Ring Closure Equations. In order to further illustrate the utility of the Z-system formalism in regard to geometric problems concerning biomolecular shapes we will now give a systematic method for solving a "bridging" or "ring closure" problem. This problem occurs in proteins at the site of a disulfide bond and in DNA at the juncture between one base pair and the next. Many algorithms for the solution of this problem have been studied and used in practice ( [33], [83]). Z-systems allow an abstract formulation and solution of the problem, which seems simpler than previous treatments.

Suppose $\Gamma^{*}$ is a Z-system on $\mathcal{N}$ describing a molecule within which two sites $r=$ $\left(A_{1}, A_{2}, A_{3}\right)$ and $r^{\prime}=\left(A_{1}^{\prime}, A_{2}^{\prime}, A_{3}^{\prime}\right)$ from $\Gamma$ are defined. We think of these two sites as being at the ends of two flexible "arms" of the molecule, and the bridging problem is to position these two arms in such a manner that two additional atoms, named $A_{0}$ and $A_{0}^{\prime}$, can be placed so as to form a bridge between $A_{1}$ and $A_{1}^{\prime}$. In addition we require that the bond lengths $l_{01}, l_{00^{\prime}}, l_{0^{\prime} 1^{\prime}}>0$ of $\left\{A_{1}, A_{0}\right\},\left\{A_{0}, A_{0}^{\prime}\right\},\left\{A_{0}^{\prime}, A_{1}^{\prime}\right\}$ are given and the bond angles $\theta_{1}, \theta_{0}, \theta_{0^{\prime}}, \theta_{1^{\prime}} \in(0, \pi)$ associated to the angles

$$
\begin{aligned}
& \left\{\left\{A_{2}, A_{1}\right\},\left\{A_{1}, A_{0}\right\}\right\}=\alpha_{1}, \\
& \left\{\left\{A_{1}, A_{0}\right\},\left\{A_{0}, A_{0}^{\prime}\right\}\right\}=\alpha_{0}, \\
& \left\{\left\{A_{0}, A_{0}^{\prime}\right\},\left\{A_{0}^{\prime}, A_{1}^{\prime}\right\}\right\}=\alpha_{0}^{\prime},
\end{aligned}
$$




$$
\left\{\left\{A_{0}^{\prime}, A_{1}^{\prime}\right\},\left\{A_{1}^{\prime}, A_{2}^{\prime}\right\}\right\}=\alpha_{1}^{\prime}
$$

are also given (see figure 12).

According to the lemma of section 3.2 there is a path $r=r_{0}, r_{1}, \ldots, r_{m}=r^{\prime}$ in the undirected site graph $\mathcal{S}(\Gamma)$ connecting $r$ to $r^{\prime}$. As was shown in sections 4.1 and 3.4 each of these edges is labeled with $\mathcal{A}_{r_{j-1}, r_{j}} \in G_{p}$ which is of the type $T_{k}$, for some $1 \leq k \leq 3$, and depends on an internal coordinate. Let $\chi$ stand for one or more parameters of which the internal coordinates appearing in this list are known functions. Let $M(\chi)=\mathcal{A}_{r_{0}, r_{1}} \mathcal{A}_{r_{1}, r_{2}} \ldots \mathcal{A}_{r_{m-1}, r_{m}}$. The matrix $M(\chi)$ is uniquely determined by the shape, and is independent of exactly which path is used to connect $r$ to $r^{\prime}$ in the undirected site graph. It can be computed numerically or symbolically by the computer program IMIMOL [22] in conjunction with the computer algebra program Maple. So for each $\chi$ let $R(\chi) \in \mathcal{B}(\Gamma)$ be such that $E_{r^{\prime}}(R(\chi))=E_{r}(R(\chi)) M(\chi)$ (our coordinatization theorem in section 4.1 guarantees the existence of $R(\chi))$.

This bridging algorithm involves three steps.

(1) Find, as functions of $\chi$ and $\sigma \in\{1,-1\}$, the position $\mathbf{R}_{A_{0}}$ of the atom $\left\{A_{0}\right\}$ relative to the configuration $R(\chi)$ such that

$$
\begin{aligned}
\left\|\mathbf{R}_{A_{0}}-\mathbf{R}_{A_{1}}(\chi)\right\| & =l_{01}, \\
\frac{\mathbf{R}_{A_{0}}-\mathbf{R}_{A_{1}}(\chi)}{\left\|\mathbf{R}_{A_{0}}-\mathbf{R}_{A_{1}}(\chi)\right\|} \cdot \frac{\mathbf{R}_{A_{2}}(\chi)-\mathbf{R}_{A_{1}}(\chi)}{\left\|\mathbf{R}_{A_{2}}(\chi)-\mathbf{R}_{A_{1}}(\chi)\right\|} & =\cos \theta_{1}, \\
\left\|\mathbf{R}_{A_{0}}-\mathbf{R}_{A_{1}^{\prime}}(\chi)\right\| & =l_{01^{\prime}}, \\
\sigma \operatorname{det}\left(\begin{array}{cccc}
1 & 1 & 1 & 1 \\
\mathbf{R}_{A_{1}}(\chi) & \mathbf{R}_{A_{2}}(\chi) & \mathbf{R}_{A_{1}^{\prime}}(\chi) & \mathbf{R}_{A_{0}}
\end{array}\right) & >0
\end{aligned}
$$

where $l_{01^{\prime}}^{2}=l_{00^{\prime}}^{2}+l_{0^{\prime} 1^{\prime}}^{2}-2 l_{00^{\prime}} l_{0^{\prime} 1^{\prime}} \cos \theta_{0^{\prime}}$.

(2) Find, as functions of $\chi$ and $\sigma^{\prime} \in\{1,-1\}$, the position $\mathbf{R}_{A_{0}^{\prime}}$ of the atom $\left\{A_{0}^{\prime}\right\}$ relative to the configuration $R(\chi)$ such that

$$
\begin{aligned}
&\left\|\mathbf{R}_{A_{0}^{\prime}}-\mathbf{R}_{A_{1}^{\prime}}(\chi)\right\|=l_{0^{\prime} 1^{\prime}}, \\
& \frac{\mathbf{R}_{A_{0}^{\prime}}-\mathbf{R}_{A_{1}^{\prime}}(\chi)}{\left\|\mathbf{R}_{A_{0}^{\prime}}-\mathbf{R}_{A_{1}^{\prime}}(\chi)\right\|} \cdot \frac{\mathbf{R}_{A_{2}^{\prime}}(\chi)-\mathbf{R}_{A_{1}^{\prime}}(\chi)}{\left\|\mathbf{R}_{A_{2}^{\prime}}(\chi)-\mathbf{R}_{A_{1}^{\prime}}(\chi)\right\|}=\cos \theta_{1^{\prime}}, \\
&\left\|\mathbf{R}_{A_{0}^{\prime}}-\mathbf{R}_{A_{1}}(\chi)\right\|=l_{0^{\prime} 1}, \\
& \sigma^{\prime} \operatorname{det}\left(\begin{array}{cccc}
1 & 1 & 1 & 1 \\
\mathbf{R}_{A_{1}^{\prime}}(\chi) & \mathbf{R}_{A_{2}^{\prime}}(\chi) & \mathbf{R}_{A_{1}}(\chi) & \mathbf{R}_{A_{0}^{\prime}}
\end{array}\right)>0
\end{aligned}
$$

where $l_{0^{\prime} 1}^{2}=l_{00^{\prime}}^{2}+l_{01}^{2}-2 l_{00^{\prime}} l_{01} \cos \theta_{0}$.

(3) For each pair $\left(\sigma, \sigma^{\prime}\right) \in\{1,-1\}^{2}$, find $\chi$ such that

$$
\left\|\mathbf{R}_{A_{0}}(\chi, \sigma)-\mathbf{R}_{A_{0}^{\prime}}\left(\chi, \sigma^{\prime}\right)\right\|=l_{00^{\prime}} .
$$

Steps (1) and (2) are completely dual to one another, and both steps entail a restriction on $\chi$ so that there are two points of intersection between a sphere and a circle. Step (3) can only be performed over those $\chi$ satisfying both restrictions.

To begin the analysis of step (1) note that the position of $A_{1}^{\prime}$ is

$$
\mathbf{R}_{A_{1}^{\prime}}(\chi)=E_{r^{\prime}}(R(\chi))\left(\begin{array}{l}
1 \\
\boldsymbol{\theta}
\end{array}\right)=E_{r}(R(\chi)) M(\chi)\left(\begin{array}{l}
1 \\
\boldsymbol{\theta}
\end{array}\right)=E_{r}(R(\chi))\left(\begin{array}{c}
1 \\
\mathbf{x}_{1}^{\prime}(\chi)
\end{array}\right),
$$


where $\left(\begin{array}{c}1 \\ \mathbf{x}_{1}^{\prime}(\chi)\end{array}\right)=M(\chi)\left(\begin{array}{l}1 \\ \boldsymbol{\theta}\end{array}\right)$ and $\mathbf{x}_{1}^{\prime}=\left(x_{1}^{\prime}, y_{1}^{\prime}, z_{1}^{\prime}\right)^{T}$. Let $\mathbf{R}_{A_{0}}=E_{r}(R(\chi))\left(\begin{array}{c}1 \\ \mathbf{x}_{0}\end{array}\right)$, where $\mathbf{x}_{0}=\left(x_{0}, y_{0}, z_{0}\right)^{T}$. We wish to solve the system of equations

$\left(l_{01} \cos \theta_{1}-x_{1}^{\prime}(\chi)\right)^{2}+\left(y_{0}-y_{1}^{\prime}(\chi)\right)^{2}+\left(z_{0}-z_{1}^{\prime}(\chi)\right)^{2}=l_{01^{\prime}}^{2}, \quad y_{0}^{2}+z_{0}^{2}=l_{01}^{2} \sin ^{2} \theta_{1}$, for $y_{0}, z_{0}$, where $x_{0}=l_{01} \cos \theta_{1}$.

Lemma. If $\left[y_{1}^{\prime}(\chi)\right]^{2}+\left[z_{1}^{\prime}(\chi)\right]^{2}>0$ then the above system of equations has at least one real solution $\left\langle y_{0}, z_{0}\right\rangle$ if and only if

$$
\begin{aligned}
\mid l_{01}^{2} \sin ^{2} \theta_{1}+\left[y_{1}^{\prime}(\chi)\right]^{2}+\left[z_{1}^{\prime}(\chi)\right]^{2} & +\left(l_{01} \cos \theta_{1}-x_{1}^{\prime}(\chi)\right)^{2}-l_{01^{\prime}}^{2} \mid \\
& \leq 2 l_{01} \sin \theta_{1} \sqrt{\left[y_{1}^{\prime}(\chi)\right]^{2}+\left[z_{1}^{\prime}(\chi)\right]^{2}}
\end{aligned}
$$

If these conditions hold then all the solutions are of the form

$$
\begin{aligned}
x_{0}= & l_{01} \cos \theta_{1}, \\
\left\langle y_{0}, z_{0}\right\rangle=\langle & \left.y_{1}^{\prime}(\chi), z_{1}^{\prime}(\chi)\right\rangle \frac{l_{01} \sin \theta_{1} \cos \alpha}{\sqrt{\left[y_{1}^{\prime}(\chi)\right]^{2}+\left[z_{1}^{\prime}(\chi)\right]^{2}}} \\
& \quad+\sigma\left\langle-z_{1}^{\prime}(\chi), y_{1}^{\prime}(\chi)\right\rangle \frac{l_{01} \sin \theta_{1} \sin \alpha}{\sqrt{\left[y_{1}^{\prime}(\chi)\right]^{2}+\left[z_{1}^{\prime}(\chi)\right]^{2}}},
\end{aligned}
$$

where $\sigma \in\{+1,-1\}$, and $\alpha \in[0, \pi]$ is such that

$$
\cos \alpha=\frac{l_{01}^{2} \sin ^{2} \theta_{1}+\left[y_{1}^{\prime}(\chi)\right]^{2}+\left[z_{1}^{\prime}(\chi)\right]^{2}+\left(l_{01} \cos \theta_{1}-x_{1}^{\prime}(\chi)\right)^{2}-l_{01^{\prime}}^{2}}{2 l_{01} \sin \theta_{1} \sqrt{\left[y_{1}^{\prime}(\chi)\right]^{2}+\left[z_{1}^{\prime}(\chi)\right]^{2}}} .
$$

Under the conditions of the above lemma we let $\mathbf{x}_{0}(\chi, \sigma)$ denote the above solution. Geometrically $\mathbf{x}_{0}(\chi, 1)$ and $\mathbf{x}_{0}(\chi,-1)$ represent the two points of intersection between a sphere (centered at $A_{1}^{\prime}$ and of radius $l_{01^{\prime}}$ ) and a circle of possible positions of $A_{0}$ (arising from fixing the distance $l_{01}$ and the angle $\theta_{1}$ ). The proof of this lemma is elementary and will be omitted. Thus we have completed step (1).

Step (2) is completely analogous to step (1); we need only exchange the roles of primed and unprimed objects. The position of $A_{1}$ is

$$
\mathbf{R}_{A_{1}}(\chi)=E_{r}(R(\chi))\left(\begin{array}{l}
1 \\
\boldsymbol{\theta}
\end{array}\right)=E_{r^{\prime}}(R(\chi)) M(\chi)^{-1}\left(\begin{array}{l}
1 \\
\boldsymbol{\theta}
\end{array}\right)=E_{r^{\prime}}(R(\chi))\left(\begin{array}{c}
1 \\
\mathbf{x}_{1}(\chi)
\end{array}\right),
$$

where $\left(\begin{array}{c}1 \\ \mathbf{x}_{1}(\chi)\end{array}\right)=M(\chi)^{-1}\left(\begin{array}{l}1 \\ \boldsymbol{\theta}\end{array}\right)$ and $\mathbf{x}_{1}=\left(x_{1}, y_{1}, z_{1}\right)^{T}$. Since $M(\chi)=\left(\begin{array}{cc}1 & \boldsymbol{\theta}^{T} \\ \mathbf{x}_{1}^{\prime}(\chi) & A(\chi)\end{array}\right)$, where $A(\chi) \in \mathrm{SO}(3)$, we have that $M(\chi)^{-1}=\left(\begin{array}{cc}1 & \boldsymbol{\theta}^{T} \\ -A(\chi)^{T} \mathbf{x}_{1}^{\prime}(\chi) & A(\chi)^{T}\end{array}\right)$, and hence $\mathbf{x}_{1}(\chi)=-A(\chi)^{T} \mathbf{x}_{1}^{\prime}(\chi)$. Let $\mathbf{R}_{A_{0}^{\prime}}=E_{r^{\prime}}(R(\chi))\left(\begin{array}{c}1 \\ \mathbf{x}_{0}^{\prime}\end{array}\right)$, where $\mathbf{x}_{0}^{\prime}=\left(x_{0}^{\prime}, y_{0}^{\prime}, z_{0}^{\prime}\right)^{T}$. Applying the analog of the above lemma we obtain solutions $\mathbf{x}_{0}^{\prime}\left(\chi, \sigma^{\prime}\right)$ under certain (additional) restrictions on $\chi$.

For step (3) note that

$$
\begin{aligned}
\mathbf{R}_{A_{0}^{\prime}}\left(\chi, \sigma^{\prime}\right) & =E_{r^{\prime}}(R(\chi))\left(\begin{array}{c}
1 \\
\mathbf{x}_{0}^{\prime}\left(\chi, \sigma^{\prime}\right)
\end{array}\right)=E_{r}(R(\chi)) M(\chi)\left(\begin{array}{c}
1 \\
\mathbf{x}_{0}^{\prime}\left(\chi, \sigma^{\prime}\right)
\end{array}\right) \\
& =E_{r}(R(\chi))\left(\begin{array}{c}
1 \\
\mathbf{x}_{1}^{\prime}(\chi)+A(\chi) \mathbf{x}_{0}^{\prime}\left(\chi, \sigma^{\prime}\right)
\end{array}\right) .
\end{aligned}
$$


Hence for each $\left(\sigma, \sigma^{\prime}\right) \in\{+1,-1\}^{2}$ the equation

$$
\begin{aligned}
l_{00^{\prime}}^{2}= & \left\|\mathbf{R}_{A_{0}}(\chi, \sigma)-\mathbf{R}_{A_{0}^{\prime}}\left(\chi, \sigma^{\prime}\right)\right\|^{2}=\left\|\mathbf{x}_{0}(\chi, \sigma)-\mathbf{x}_{1}^{\prime}(\chi)-A(\chi) \mathbf{x}_{0}^{\prime}\left(\chi, \sigma^{\prime}\right)\right\|^{2} \\
= & l_{01}^{2}+l_{0^{\prime} 1^{\prime}}^{2}+\left\|\mathbf{x}_{1}^{\prime}(\chi)\right\|^{2}-2 \mathbf{x}_{0}(\chi, \sigma) \cdot \mathbf{x}_{1}^{\prime}(\chi)-2 \mathbf{x}_{0}(\chi, \sigma) \cdot A(\chi) \mathbf{x}_{0}^{\prime}\left(\chi, \sigma^{\prime}\right) \\
& +2 \mathbf{x}_{1}^{\prime}(\chi) \cdot A(\chi) \mathbf{x}_{0}^{\prime}\left(\chi, \sigma^{\prime}\right)
\end{aligned}
$$

must be solved for $\chi$ also satisfying the pair of restrictions

$$
\begin{aligned}
\mid l_{01}^{2} \sin ^{2} \theta_{1}+\left[y_{1}^{\prime}(\chi)\right]^{2}+\left[z_{1}^{\prime}(\chi)\right]^{2} & +\left(l_{01} \cos \theta_{1}-x_{1}^{\prime}(\chi)\right)^{2}-l_{01^{\prime}}^{2} \mid \\
& \leq 2 l_{01} \sin \theta_{1} \sqrt{\left[y_{1}^{\prime}(\chi)\right]^{2}+\left[z_{1}^{\prime}(\chi)\right]^{2}} \\
\mid l_{0^{\prime} 1^{\prime}}^{2} \sin ^{2} \theta_{1}^{\prime}+\left[y_{1}(\chi)\right]^{2}+\left[z_{1}(\chi)\right]^{2} & +\left(l_{0^{\prime} 1^{\prime}} \cos \theta_{1}^{\prime}-x_{1}(\chi)\right)^{2}-l_{0^{\prime} 1}^{2} \mid \\
\leq & 2 l_{0^{\prime} 1^{\prime}} \sin \theta_{1}^{\prime} \sqrt{\left[y_{1}(\chi)\right]^{2}+\left[z_{1}(\chi)\right]^{2}}
\end{aligned}
$$

as well as $\left[y_{1}^{\prime}(\chi)\right]^{2}+\left[z_{1}^{\prime}(\chi)\right]^{2}>0$ and $\left[y_{1}(\chi)\right]^{2}+\left[z_{1}(\chi)\right]^{2}>0$.

Lemma. This method finds all solutions of the bridging problem in the parameterized family $R(\chi)$ for all $\chi$ such that the simplices $\left\{\mathbf{R}_{A_{1}}(\chi), \mathbf{R}_{A_{2}}(\chi), \mathbf{R}_{A_{1}^{\prime}}(\chi)\right\}$ and $\left\{\mathbf{R}_{A_{1}^{\prime}}(\chi), \mathbf{R}_{A_{2}^{\prime}}(\chi), \mathbf{R}_{A_{1}}(\chi)\right\}$ are non-collinear.

Proof. Clearly any solution of the bridging problem in the parameterized family $R(\chi)$ with the simplices $\left\{\mathbf{R}_{A_{1}}, \mathbf{R}_{A_{2}}, \mathbf{R}_{A_{1}^{\prime}}\right\}$ and $\left\{\mathbf{R}_{A_{1}^{\prime}}, \mathbf{R}_{A_{2}^{\prime}}, \mathbf{R}_{A_{1}}\right\}$ being non-collinear must satisfy $\left[y_{1}^{\prime}(\chi)\right]^{2}+\left[z_{1}^{\prime}(\chi)\right]^{2}>0$ and $\left[y_{1}(\chi)\right]^{2}+\left[z_{1}(\chi)\right]^{2}>0$, and all the other equations and inequalities in our method. Conversely, any $\chi$ obtained from our method gives rise to a solution of the bridging problem: most of the constraints are applied directly, but the angle constraints at $\alpha_{0}$ and $\alpha_{0}^{\prime}$ are imposed indirectly via the distance constraints on $l_{01^{\prime}}$ and $l_{0^{\prime} 1}$.

6.7. Hexagons with two-fold symmetry. As an application of our bridging algorithm and as a further illustration of the systematic procedure for solving geometric problems made possible by the Z-system formalism we will study closed six-sided polygons in $\mathbb{R}^{3}$ with fixed bond lengths and bond angles. For simplicity we will assume a two-fold rotational symmetry is present in these lengths and angles, i.e. opposite sides are of equal length and opposite angles are of equal measure; see figure 13. Well-known chemical examples of this situation are the molecules cyclohexane and cyclohexanedione ( [18], [19], [79]). We will derive a single equation which will allow the classification of almost all the shapes of the hexagon. Some of the solutions of this equation represent "flexible" hexagons, whereas the others represent "rigid" hexagons. The existence of flexible hexagons in the generality presented here is a theorem in the kinematics [68] of six-membered rings and of octahedra first proved by Bricard [15] (see also [53], and especially [13]).

Consider a hexagon whose vertices have names from the set $\mathcal{N}^{\prime}=\left\{A_{0}, A_{1}, A_{2}\right.$, $\left.A_{0}^{\prime}, A_{1}^{\prime}, A_{2}^{\prime}\right\}$, as in figure 13. Let $\mathcal{N}=\left\{A_{0}, A_{1}, A_{2}, A_{0}^{\prime}\right\}$ and the Z-system $\Gamma^{*}$ on $\mathcal{N}$ be defined as

$$
\begin{aligned}
& \Gamma^{1}=\left\{\left\{A_{0}, A_{1}\right\},\left\{A_{1}, A_{2}\right\},\left\{A_{2}, A_{0}^{\prime}\right\}\right\}, \\
& \Gamma^{2}=\left\{\left\{A_{0}, A_{1}, A_{2}\right\},\left\{A_{1}, A_{2}, A_{0}^{\prime}\right\}\right\}, \\
& \Gamma_{*}^{3}=\left\{\left[A_{0}, A_{1}, A_{2}, A_{0}^{\prime}\right]\right\} .
\end{aligned}
$$

The (given) labels of the bonds in $\Gamma^{1}$ are $l_{0}, l_{1}, l_{2}$ respectively, and the (given) labels of the angles in $\Gamma^{2}$ are $\cos \theta_{1}, \cos \theta_{2}$ respectively. Let the (unknown) label of 


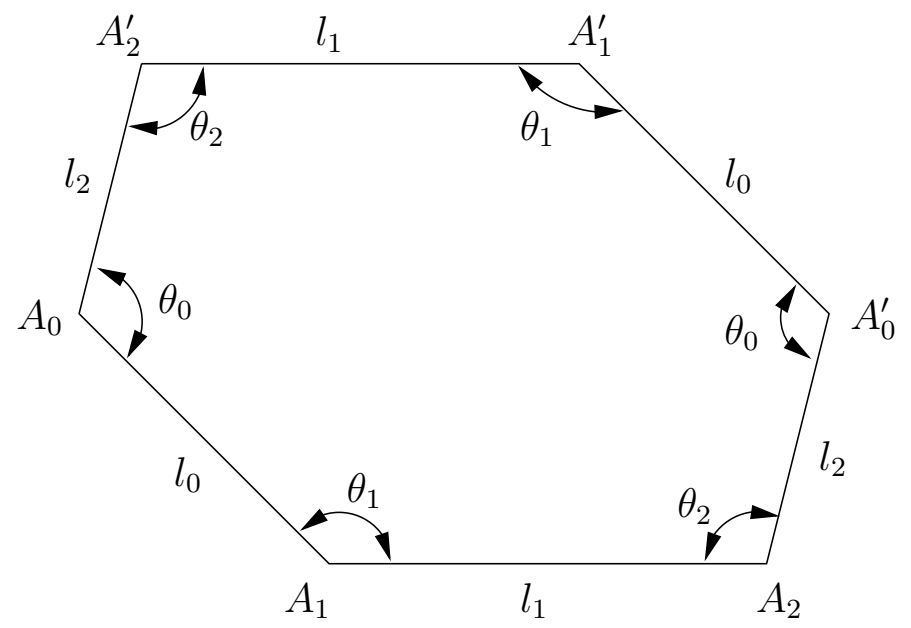

Figure 13. A symmetric hexagon with fixed lengths $l_{0}, l_{1}, l_{2}$ and fixed angles $\theta_{0}, \theta_{1}, \theta_{2}$.

$e^{*}=\left[A_{0}, A_{1}, A_{2}, A_{0}^{\prime}\right]$ in $\Gamma_{*}^{3}$ be $e^{i \varphi_{1}} \cdot \varphi_{1}$ plays the role of $\chi$ of the bridging algorithm. Let sites $r=\left(A_{0}, A_{1}, A_{2}\right)$ and $r^{\prime}=\left(A_{0}^{\prime}, A_{2}, A_{1}\right)$ from the Z-system be given. A path in the site graph $\mathcal{S}\left(\Gamma^{*}\right)$ from $r$ to $r^{\prime}$, together with the label from $G_{p}$ of each edge is given by the following.

$$
\begin{aligned}
& \left(A_{0}, A_{1}, A_{2}\right) \\
& \times \quad T_{1}\left(l_{0}\right) \\
& \left(A_{1}, A_{0}, A_{2}\right) \\
& \times \quad T_{2}\left(\cos \theta_{1}\right) \\
& \left(A_{1}, A_{2}, A_{0}\right) \\
& \downarrow \quad T_{3}\left(e^{i \varphi_{1}}\right) \\
& \left(A_{1}, A_{2}, A_{0}^{\prime}\right) \\
& \times \\
& T_{1}\left(l_{1}\right) \\
& \left(A_{2}, A_{1}, A_{0}^{\prime}\right) \\
& \times \\
& T_{2}\left(\cos \theta_{2}\right) \\
& \left(A_{2}, A_{0}^{\prime}, A_{1}\right) \\
& x \\
& T_{1}\left(l_{2}\right) \\
& \left(A_{0}^{\prime}, A_{2}, A_{1}\right)
\end{aligned}
$$

Define $M\left(\varphi_{1}\right)=T_{1}\left(l_{0}\right) T_{2}\left(\cos \theta_{1}\right) T_{3}\left(e^{i \varphi_{1}}\right) T_{1}\left(l_{1}\right) T_{2}\left(\cos \theta_{2}\right) T_{1}\left(l_{2}\right)$. Matrix products like these should be performed using computer algebra software, such as Maple. The program IMIMOL allows the user to define two sites and export a Maple procedure for computing this matrix product, since the path in the site graph connecting the two sites can be generated algorithmically (see section 3.2), and edges of trees in the Z-system can be labeled (in IMIMOL) with variable names (any string) as well as with numbers [22]. 
We will use a Z-system $\Gamma^{\prime}$ on $\mathcal{N}^{\prime}$ to describe shapes of the symmetric hexagon. This Z-system is defined by

$$
\begin{aligned}
& \Gamma^{\prime 1}=\Gamma^{1} \cup\left\{\left\{A_{0}, A_{2}^{\prime}\right\},\left\{A_{0}^{\prime}, A_{1}^{\prime}\right\}\right\}, \\
& {\Gamma^{\prime}}^{2}=\Gamma^{2} \cup\left\{\left\{A_{2}^{\prime}, A_{0}, A_{1}\right\},\left\{A_{1}^{\prime}, A_{0}^{\prime}, A_{2}\right\}\right\}, \\
& {\Gamma^{\prime}}^{3}=\Gamma_{*}^{3} \cup\left\{\left[A_{2}^{\prime}, A_{0}, A_{1}, A_{2}\right],\left[A_{1}, A_{2}, A_{0}^{\prime}, A_{1}^{\prime}\right]\right\} .
\end{aligned}
$$

The labeling of $\Gamma^{\prime}$ will be an extension of that of $\Gamma$. The labels of the two new bonds are $l_{2}, l_{0}$ respectively, and the labels of the two new angles are both $\cos \theta_{0}$. The (unknown) labels of the two new wedges will be denoted by $e^{i \varphi_{0}}$ and $e^{i \varphi_{2}}$ respectively. By a "configuration of the symmetric hexagon" we will mean a configuration $R: \mathcal{N}^{\prime} \rightarrow \mathbb{R}^{3}$ such that $\hat{\eta}\left(G_{a} R\right)$ is the above labeling of the Z-system $\Gamma^{\prime}$ (containing three unknown variables $\left.\varphi_{0}, \varphi_{1}, \varphi_{2}\right)$ such that the distance between $A_{1}^{\prime}$ and $A_{2}^{\prime}$ is $l_{1}$ and the angles at $A_{1}^{\prime}$ and $A_{2}^{\prime}$ are $\theta_{1}$ and $\theta_{2}$ respectively, as indicated in figure 13 .

If we apply our three step bridging method we obtain the following.

Theorem. Suppose $l_{0}, l_{1}, l_{2}>0$ and $0<\theta_{0}, \theta_{1}, \theta_{2}<\pi$. Define

$$
\begin{aligned}
a_{0}: & =l_{1}^{2}+l_{2}^{2}-2 l_{1} l_{2} \cos \theta_{2} \\
a_{1}: & =l_{0}^{2}+l_{2}^{2}-2 l_{0} l_{2} \cos \theta_{0} \\
a_{2}: & =l_{0}^{2}+l_{1}^{2}-2 l_{0} l_{1} \cos \theta_{1} \\
b_{0}: & =\cos \theta_{1} \cos \theta_{2}-\cos \theta_{0} \\
b_{1}: & =\cos \theta_{0} \cos \theta_{2}-\cos \theta_{1} \\
b_{2}: & =\cos \theta_{0} \cos \theta_{1}-\cos \theta_{2} \\
c: & =a_{1} b_{0}+l_{0} l_{2} \sin ^{2} \theta_{0}+l_{1}\left(l_{2} b_{1}+l_{0} b_{2}\right) \\
& =\frac{\left(l_{2}^{2}-a_{2}\right)\left(l_{0}^{2}-a_{0}\right)+\left(a_{1}-l_{1}^{2}\right)\left[a_{1}+\left(l_{1}-2 l_{0} \cos \theta_{1}\right)\left(l_{1}-2 l_{2} \cos \theta_{2}\right)\right]}{} \\
d: & =l_{1}^{2} l_{2}^{2} \sin ^{2} \theta_{2}+l_{0}^{2} l_{2}^{2} \sin ^{2} \theta_{0}+l_{0}^{2} l_{1}^{2} \sin ^{2} \theta_{1}+2 l_{0} l_{1} l_{2}\left[l_{0} b_{2}+l_{1} b_{0}+l_{2} b_{1}\right] \\
& =\frac{1}{4}\left(a_{0}+a_{1}+a_{2}\right)^{2}-\frac{1}{2}\left(a_{0}^{2}+a_{1}^{2}+a_{2}^{2}\right) \\
Y: & =\sin \theta_{1} \sin \theta_{2} \cos \varphi_{1} \\
K: & =a_{0}-\left(l_{1} \cos \theta_{1}-l_{2} \cos \theta_{1} \cos \theta_{2}+l_{2} Y\right)^{2} \\
K^{\prime}: & =a_{2}-\left(l_{1} \cos \theta_{2}-l_{0} \cos \theta_{1} \cos \theta_{2}+l_{0} Y\right)^{2} .
\end{aligned}
$$

Suppose $d \geq 0$ and $\varphi_{1}$ satisfies $K>0, K^{\prime}>0$, and

$$
\frac{c-\sin \theta_{0} \sqrt{d}}{a_{1} \sin \theta_{1} \sin \theta_{2}} \leq \cos \varphi_{1} \leq \frac{c+\sin \theta_{0} \sqrt{d}}{a_{1} \sin \theta_{1} \sin \theta_{2}}
$$

Let $\left(\sigma, \sigma^{\prime}\right) \in\{+1,-1\}^{2}$. Suppose there exists a configuration $R: \mathcal{N}^{\prime} \rightarrow \mathbb{R}^{3}$ of the symmetric hexagon such that the sets $\left\{\mathbf{R}_{A_{0}}, \mathbf{R}_{A_{1}}, \mathbf{R}_{A_{0}^{\prime}}\right\}$ and $\left\{\mathbf{R}_{A_{0}^{\prime}}, \mathbf{R}_{A_{2}}, \mathbf{R}_{A_{0}}\right\}$ are not collinear and

$$
\sigma \operatorname{det}\left(\begin{array}{cccc}
1 & 1 & 1 & 1 \\
\mathbf{R}_{A_{0}} & \mathbf{R}_{A_{1}} & \mathbf{R}_{A_{0}^{\prime}} & \mathbf{R}_{A_{2}^{\prime}}
\end{array}\right) \geq 0, \quad \sigma^{\prime} \operatorname{det}\left(\begin{array}{cccc}
1 & 1 & 1 & 1 \\
\mathbf{R}_{A_{0}^{\prime}} & \mathbf{R}_{A_{2}} & \mathbf{R}_{A_{0}} & \mathbf{R}_{A_{1}^{\prime}}
\end{array}\right) \geq 0
$$

Then one of the following conditions holds.

(1) $1+\sigma \sigma^{\prime}=0$.

(2) $Y-b_{0}=0$, i.e. $\cos \varphi_{1}=\frac{b_{0}}{\sin \theta_{1} \sin \theta_{2}}$.

(3) $a_{1}-l_{1}^{2}=0$ and $\left(l_{2}^{2}-a_{2}\right)\left(l_{0}^{2}-a_{0}\right)=0$. 
(4) $a_{1} \neq l_{1}^{2}$ and $\left(a_{1}-l_{1}^{2}\right)\left(Y+b_{0}\right)-2 c=0$, i.e. $\cos \varphi_{1}=\frac{2 c-b_{0}\left(a_{1}-l_{1}^{2}\right)}{\left(a_{1}-l_{1}^{2}\right) \sin \theta_{1} \sin \theta_{2}}$.

In any of these cases $\varphi_{0}$ and $\varphi_{2}$ are explicit functions of $\varphi_{1}$ as follows:

$$
\begin{aligned}
& \varphi_{0}=\arg \left[\frac{l_{1} \sin \theta_{1}-l_{2}\left(\sin \theta_{1} \cos \theta_{2}+\cos \theta_{1} \sin \theta_{2} \cos \varphi_{1}\right)-i l_{2} \sin \theta_{2} \sin \varphi_{1}}{\sqrt{K}}\right]-\sigma \alpha \\
& \varphi_{2}=\arg \left[\frac{l_{1} \sin \theta_{2}-l_{0}\left(\cos \theta_{1} \sin \theta_{2}+\sin \theta_{1} \cos \theta_{2} \cos \varphi_{1}\right)-i l_{0} \sin \theta_{1} \sin \varphi_{1}}{\sqrt{K^{\prime}}}\right]-\sigma^{\prime} \alpha^{\prime}
\end{aligned}
$$

where $\alpha, \alpha^{\prime} \in[0, \pi]$ are such that

$$
\begin{aligned}
\cos \alpha & =\frac{\left(l_{2} \cos \theta_{0}-l_{0}\right)\left(Y-b_{0}\right)+l_{1} b_{2}+l_{2} \sin ^{2} \theta_{0}}{\sin \theta_{0} \sqrt{K}} \\
\cos \alpha^{\prime} & =\frac{\left(l_{0} \cos \theta_{0}-l_{2}\right)\left(Y-b_{0}\right)+l_{1} b_{1}+l_{0} \sin ^{2} \theta_{0}}{\sin \theta_{0} \sqrt{K^{\prime}}}
\end{aligned}
$$

Proof. We always have $K \geq 0$, and $K=0$ if and only if $\left\{\mathbf{R}_{A_{0}}, \mathbf{R}_{A_{1}}, \mathbf{R}_{A_{0}^{\prime}}\right\}$ is collinear. A similar assertion holds for $K^{\prime}$ and $\left\{\mathbf{R}_{A_{0}^{\prime}}, \mathbf{R}_{A_{2}}, \mathbf{R}_{A_{0}}\right\}$. Because of the two-fold symmetry of the hexagon the two inequality restrictions required in the bridging algorithm coincide with the restriction on $\cos \phi_{1}$ in the statement of the theorem. This is because the quantities $c, d, a_{1}$, etc. involved in the upper and lower limits for $\cos \phi_{1}$ are invariant under the replacements $l_{0} \mapsto l_{2}, l_{1} \mapsto l_{1}, l_{2} \mapsto$ $l_{0}, \theta_{0} \mapsto \theta_{0}, \theta_{1} \mapsto \theta_{2}, \theta_{2} \mapsto \theta_{1}$, which correspond to switching the roles of primed and unprimed variables in the bridging algorithm.

The main equation to be solved for $\phi_{1}$ (step (3) of the bridging algorithm) involves both the sine and cosine of the angles $\alpha$ and $\alpha^{\prime}$. Especially $\sin \alpha$ and $\sin \alpha^{\prime}$ are complicated functions of $\phi_{1}$. A crucial identity which allows us to simplify the main equation is: $K \sin ^{2} \alpha=K^{\prime} \sin ^{2} \alpha^{\prime}$. This verification, as well as most of the others of this proof, are best done using a computer algebra system like Maple. Define $Q=K \sin ^{2} \alpha \sin ^{2} \theta_{0}$ and $Y=\sin \theta_{1} \sin \theta_{2} \cos \phi_{1}$. It follows that

$$
\begin{aligned}
Q & =-a_{1} Y^{2}+2 c Y-2 b_{0} c+\left(a_{1}-l_{1}^{2}\right) b_{0}^{2}+l_{1}^{2} \sin ^{2} \theta_{1} \sin ^{2} \theta_{2} \\
& =-a_{1}\left(Y-\frac{c-\sin \theta_{0} \sqrt{d}}{a_{1}}\right)\left(Y-\frac{c+\sin \theta_{0} \sqrt{d}}{a_{1}}\right) .
\end{aligned}
$$

The inequality restriction on $\cos \phi_{1}$, or better on $Y$, is exactly that $Q \geq 0$. The main equation to be solved can be written as $C_{1}(Y) \sqrt{Q}=C_{2}(Y)$, where $C_{1}(Y), C_{2}(Y)$ are polynomials in $Y$ with coefficients which are polynomials in $l_{0}, l_{1}, l_{2}, \sigma, \sigma^{\prime}$, and the sines and cosines of $\theta_{0}, \theta_{1}, \theta_{2}$. We must square both sides of this equation, keeping in mind the possibility of thereby introducing extraneous solutions. What results is a polynomial in $Y$ of degree 8 , and we desire its roots. Remarkably it factors (up to trivial non-vanishing quantities) as follows:

$$
\left(1+\sigma \sigma^{\prime}\right)\left(Y-b_{0}\right)\left[\left(a_{1}-l_{1}^{2}\right)\left(Y+b_{0}\right)-2 c\right] Q K K^{\prime}=0 .
$$

(This must be checked using Maple; the expression has over 24000 terms.) Alternatives (1) and (2) in the statement of the theorem are obviously arising from the first two factors. Alternative (3) is the only way the third factor can vanish for all $Y$. Alternative (4) is the only $Y$ dependent way the third factor can vanish. When $Q=0$ we have $\sin \alpha=0$ and $\sin \alpha^{\prime}=0$ (and hence the two determinants in the statement of the theorem also vanish) so the values of $\left(\sigma, \sigma^{\prime}\right)$ do not matter, and the solutions are already included in alternative (1). The last two factors $K, K^{\prime}$ 
are positive by assumption. Hence the four listed alternatives exhaust all possible solutions.

Several remarks about this theorem are in order. First of all in the classical case of cyclohexane $l_{0}=l_{1}=l_{2}=1, \theta_{0}=\theta_{1}=\theta_{2}=\cos ^{-1}\left(-\frac{1}{3}\right)$, the parameterized curves $\left\{\left(\phi_{0}\left(\phi_{1}, \sigma\right), \phi_{1}, \phi_{2}\left(\phi_{1}, \sigma^{\prime}\right)\right)|| \phi_{1} \mid \leq \cos ^{-1}\left(\frac{10-3 \sqrt{6}}{8}\right)\right\}$ for $\left(\sigma, \sigma^{\prime}\right)=(1,-1)$ or $(-1,1)$ (i.e. alternative $(1))$ fit together to form a smooth closed curve corresponding to the well-known one-parameter "twist/boat" family of solutions. Alternative (2) yields either $\left(\phi_{1}, \sigma, \sigma^{\prime}\right)=(\pi / 3,1,1)$ or $(-\pi / 3,-1,-1)$, which are the well-known rigid "chair" solutions. The other two possibilities $(\pi / 3,-1,-1)$ or $(-\pi / 3,1,1)$ are extraneous. Alternative (3) does not occur, and the value for $\cos \phi_{1}$ in alternative (4) is out of the allowed range. Hence the classically known solutions are the only solutions in this case.

Alternative (1) corresponds to Bricard's first family of flexible octahedra [15], although we do not assert that any $\left(\phi_{1}, \sigma, \sigma^{\prime}\right)$ satisfying alternative (1) is a (nonextraneous) solution. We however have no example where a $\left(\phi_{1}, \sigma, \sigma^{\prime}\right)$ satisfying alternative (1) fails to be a solution. Alternative (3) corresponds to the intersection of Bricard's first and second families of flexible octahedra [15], and when $\sigma \sigma^{\prime}=1$ yields a distinct family of solutions from alternative (1), where $\sigma \sigma^{\prime}=-1$. An example where alternative (3) solutions occur is $l_{0}=1, l_{1}=\sqrt{2}, l_{2}=1, \theta_{0}=\theta_{1}=$ $\pi / 2, \theta_{2}=\pi / 4$. When $\left(\sigma, \sigma^{\prime}\right)=(1,1)$ the $\phi_{1}$ interval $[-\pi, 0]$ gives actual solutions whereas the interval $(0, \pi)$ gives extraneous solutions, except at $\phi_{1}=\pi / 2$ which is an alternative $(2)$ rigid solution. When $\left(\sigma, \sigma^{\prime}\right)=(-1,-1)$ the $\phi_{1}$ interval $(-\pi, 0)$ gives extraneous solutions except at $\phi_{1}=-\pi / 2$, which is an alternative (2) rigid solution; the $\phi_{1}$ interval $[0, \pi]$ gives actual solutions. These two parameterized curves of actual solutions fit together to form a smooth closed curve in $\left(\phi_{0}, \phi_{1}, \phi_{2}\right)$ space. Alternative (1) yields two additional closed curves of solutions. One is given by $\left(\sigma, \sigma^{\prime}\right)=(1,-1), \phi_{1} \in[-\pi, 0]$ together with $\left(\sigma, \sigma^{\prime}\right)=(-1,1), \phi_{1} \in[0, \pi]$. The other is given by $\left(\sigma, \sigma^{\prime}\right)=(-1,1), \phi_{1} \in[-\pi, 0]$ together with $\left(\sigma, \sigma^{\prime}\right)=(1,-1), \phi_{1} \in[0, \pi]$. All three closed curves pass through the two points $\left(\phi_{0}, \phi_{1}, \phi_{2}\right)=(\pi, 0,0),(0, \pi, 0)$, where $Q=0$. Both $K$ and $K^{\prime}$ remain positive on all three curves. Alternative (4) is impossible in this example.

Another interesting example is $l_{0}=l_{1}=l_{2}=1, \theta_{0}=\pi / 4, \theta_{1}=\theta_{2}=\pi / 2$. Alternative (1) gives rise to two disjoint smooth closed curves of actual solutions in $\left(\phi_{0}, \phi_{1}, \phi_{2}\right)$ space, corresponding to the two possibilities $\left(\sigma, \sigma^{\prime}\right)=(1,-1)$ or $(-1,1)$. Alternative (2) solutions are $\left(\phi_{0}, \phi_{1}, \phi_{2}\right)=(\pi / 2,-3 \pi / 4, \pi / 2)$ (when $\left.\left(\sigma, \sigma^{\prime}\right)=(-1,-1)\right)$ and $\left(\phi_{0}, \phi_{1}, \phi_{2}\right)=(-\pi / 2,3 \pi / 4,-\pi / 2)\left(\right.$ when $\left.\left(\sigma, \sigma^{\prime}\right)=(1,1)\right)$, the other two possibilities being extraneous. Alternative (3) is impossible, but alternative (4) gives two additional rigid solutions: $\left(\phi_{0}, \phi_{1}, \phi_{2}\right)=\left(34.06^{\circ}, 72.97^{\circ}, 34.06^{\circ}\right)$ (when $\left.\left(\sigma, \sigma^{\prime}\right)=(-1,-1)\right)$ and $\left(\phi_{0}, \phi_{1}, \phi_{2}\right)=\left(-34.06^{\circ},-72.97^{\circ},-34.06^{\circ}\right)$ (when $\left.\left(\sigma, \sigma^{\prime}\right)=(1,1)\right)$, the other two possibilities being extraneous. The existence of rigid solutions arising from alternative (4) seems to have been missed in [13], and they in fact yield counterexamples to several of the assertions in that paper. For example it is claimed that for rigid solutions the three lines determined by the pairs $\left\{\mathbf{R}_{A_{0}}, \mathbf{R}_{A_{0}^{\prime}}\right\},\left\{\mathbf{R}_{A_{1}}, \mathbf{R}_{A_{1}^{\prime}}\right\}$, and $\left\{\mathbf{R}_{A_{2}}, \mathbf{R}_{A_{2}^{\prime}}\right\}$ all intersect in a single point of space. In the above example these three lines behave as claimed for the alternative (2) solutions but the three lines are distinct and parallel in the alternative (4) solutions. 
It is easy to show that $Q=l_{1}^{2}\left[\sin ^{2} \theta_{1} \sin ^{2} \theta_{2}-Y^{2}\right]$ if and only if we are in alternative (2) or in alternative (4). Thus in either of these cases we have $\sqrt{Q}=$ $l_{1} \sin \theta_{1} \sin \theta_{2}\left|\sin \phi_{1}\right|$. One can verify (again using Maple) that $\left(\mathbf{R}_{A_{0}^{\prime}}-\mathbf{R}_{A_{0}}\right) \times$ $\left(\mathbf{R}_{A_{2}^{\prime}}-\mathbf{R}_{A_{2}}\right)=\boldsymbol{\theta}$ under the assumption that the pose at the site $\left(A_{0}, A_{1}, A_{2}\right)$ is $\left(\boldsymbol{\theta}, \hat{\mathbf{e}}_{1}, \hat{\mathbf{e}}_{2}, \hat{\mathbf{e}}_{3}\right)$, and for a solution from alternative (4) with $\sigma\left|\sin \phi_{1}\right|=-\sin \phi_{1}$. By symmetry we also have $\left(\mathbf{R}_{A_{0}^{\prime}}-\mathbf{R}_{A_{0}}\right) \times\left(\mathbf{R}_{A_{1}^{\prime}}-\mathbf{R}_{A_{1}}\right)=\boldsymbol{\theta}$. Thus alternative (4) solutions of this type always have the three lines passing through pairs of opposite vertices being parallel. For this reason we could call them hexagons on a triangular cylinder. Such hexagons can have one crossing (i.e. a pair of opposite sides intersect) or three crossings. The example in the previous paragraph has one crossing. An example with three crossings is $l_{0}=l_{1}=l_{2}=1, \theta_{0}=\theta_{1}=\theta_{2}=\pi / 4,\left(\phi_{0}, \phi_{1}, \phi_{2}\right)=$ $\left(40.768^{\circ},-40.768^{\circ}, 40.768^{\circ}\right)$ when $\left(\sigma, \sigma^{\prime}\right)=(1,1)$. It is possible to make arbitrarily small perturbations of this last example (into the family of hexagons which have a three-fold symmetry instead of the two-fold symmetric hexagons we have studied in this section) which form trefoil knots [79].

Models for all the examples discussed in this section can be constructed using the set of building toys called "K'nex". The bond angles occurring in most of these examples are too small to be chemically relevant. Nevertheless we have demonstrated the utility of the Z-system formalism introduced in this paper when coupled with the power of computer algebra software such as Maple.

Acknowledgments: The author offers his thanks to P. G. Wolynes for extending him hospitality and for many useful conversations during his sabbatical at the University of Illinois. Thanks also to Zan Schulten, Todd Martinez, Tom Hughes, and Vassiliy Lubchenko for many stimulating conversations. The author has enjoyed many discussions with his colleagues László Székely, Ralph Howard, David Sumner, Xian Wu and Lukasz Lebioda about various parts of this work. The author appreciates the direct and indirect financial support of the Industrial Mathematics Institute (IMI) at the University of South Carolina. Thanks also to André Dreiding and Wilma Olson for help with references.

\section{REFERENCES}

[1] E. Abillon, Geometry of the five-membered ring. Mathematical demonstration of the pseudorotation formulae, Biophysics of Structure and Mechanism, 8, 257-270, 1982.

[2] R. Abraham, J.E. Marsden, Foundations of Mechanics, second edition, Benjamin/Cummings, London, 1978.

[3] W.J. Adams, H.J. Geise, L.S. Bartell, Structure, Equilibrium Conformation, and Pseudorotation in Cyclopentane. An Electron Diffraction Study, J. Am. Chem. Soc., 92, no. 17, 5013-5019, 1970.

[4] P.S. Aleksandrov, Combinatorial Topology, Graylock Press, Rochester, New York, 1956.

[5] D.G. Babbitt, V.S. Varadarajan, Local Moduli for Meromorphic Differential Equations, Astérisque, 169-170, 1989.

[6] J. Baker, A. Kessi, B. Delley, The generation and use of delocalized internal coordinates in geometry optimization, J. Chem. Phys., 105, no. 1, 192-212, 1 Jul 1996.

[7] J. Baker, D. Kinghorn, P. Pulay, Geometry optimization in delocalized internal coordinates: An efficient quadratically scaling algorithm for large molecules, J. Chem. Phys., 110, no. 11, 4986-4991, 15 Mar 1999.

[8] A.T. Balaban (Ed.), Chemical Applications of Graph Theory, Academic Press, London, 1976.

[9] A.T. Balaban (Ed.), From Chemical Topology to Three-Dimensional Geometry, Plenum Press, New York, 1997.

[10] M. Berger, Geometry, Springer-Verlag, Berlin, 1987.

[11] G.M. Blackburn, M.J. Gait, (eds.), Nucleic Acids in Chemistry and Biology, IRL Press, Oxford, 1990. 
[12] J. Bochnak, M. Coste, M-F. Roy, Real Algebraic Geometry, Springer, Berlin, 1998.

[13] O. Bottema, Flexible Hexagons, Nederl. Akad. Wetensch. Proc. Ser. A 70 (i.e. Indagationes Mathematicae, 29), 151-155, 1967.

[14] C. Brandon, J. Tooze, Introduction to Protein Structure, Garland Publishing, Inc., New York, 1991.

[15] R. Bricard, Mémoire sur la thérie de l'octaèdre articulé, Journal de Mathématiques Pures et Appliqées, 5, no. 3, 113-148, 1897.

[16] T. Carrington, Jr., Vibrational Energy Level Calculations, in the Encyclopedia of Computational Chemistry, (P.v.R. Schleyer, ed.), Volume 5, 3157-3166, John Wiley and Sons, Chichester, 1998.

[17] Cerius² Users Guide, Quantum Mechanics, Quantum Chemistry, ADF, Gaussian, MOPAC, Molecular Simulations, Inc., San Diego, 1997.

[18] G.M. Crippen, T.F. Havel, Distance Geometry and Molecular Conformation, Research Studies Press, Taunton, Somerset, England, 1988.

[19] G.M. Crippen, Exploring the Conformation Space of Cycloalkanes by Linearized Embedding, J. Computational Chem., 13, no. 3, 351-361, 1992.

[20] X. Chapuisat, C. Iung, Vector Parameterization of the N-Body Problem in Quantum Mechanics: Polyspherical Coordinates, Physical Review A, Vol. 45, No. 9, 6217-6235, 1992.

[21] G.K. Das, D. Bhattacharyya, D.B. Burma, A Possible Mechanism of Peptide Bond Formation on Ribosome without Mediation of Peptidyl Transferase, J. Theor. Biol., 200, 193-205, 1999.

[22] D. Dix, IMIMOL: A Computer Program for Molecular Geometry Specification and Computation, 2004, http://www.math.sc.edu/ $\sim \operatorname{dix} /$ imimol.pdf.

[23] A. Dreiding, K. Wirth, The Multiplex: A Classification of Finite Ordered Point Sets in Oriented d-Dimensional Spaces, Match, No. 8, 341-352, 1980.

[24] A. Dress, A. Dreiding, H. Haegi, Classification of Mobile Molecules by Category Theory, in Symmetries and Properties of Non-Rigid Molecules: A Comprehensive Survey, J. Maruani and J. Serre (eds.), Studies in Physical and Theoretical Chemistry, Vol. 23, 39-58, Elsevier, Amsterdam, 1983.

[25] D. Dufus, I. Rival, M. Simonovits, Spanning retracts of a partially ordered set, Discrete Math., 32,1-7, 1980.

[26] I.Z. Emiris, B. Mourrain, Computer Algebra Methods for Studying and Computing Molecular Conformations, Algorithmica, 25, 372-402, 1999.

[27] E. Estrada, Characterization of $3 D$ molecular structure, Chem. Phys. Lett., 319, no. 5-6, 713-718, 2000.

[28] H. Eyring, The Resultant Electric Moment of Complex Molecules, Phys. Rev., 39, 746-748, 1932.

[29] J.B. Foresman, A. Frisch, Exploring Chemistry with Electronic Structure Methods, second edition, Gaussian, Inc., Pittsburgh, 1996.

[30] B. Fuglede, Integration on the nth power of a hyperbolic space in terms of invariants under diagonal action of isometries (Lorentz transformations), Commun. Math. Phys., Vol. 129, No. 3, 481-509, 1990.

[31] J.H. Frederick, C. Woywod, General Formulation of the Vibrational Kinetic Energy Operator in Internal Bond-Angle Coordinates, J. Chem. Phys., Vol. 111, No. 16, 7255-7271, 1999.

[32] F. Gatti, C. Muñoz, C. Iung, A General Expression of the Kinetic Energy Operator in Polyspherical Coordinates, J. Chem. Phys., 114, no. 19, 8275-8281, 2001.

[33] N. Gō, H.A. Scheraga, Ring Closure and Local Conformational Deformations of Chain Molecules, Macromolecules, 3, no. 2, 178-187, 1970.

[34] G.H. Golub, C.F. Van Loan, Matrix Computations, The Johns Hopkins University Press, Baltimore, 1996.

[35] J.E. Goodman, J. O'Rourke, eds. Handbook of Discrete and Computational Geometry, CRC Press, Boca Raton, Florida, 1997.

[36] J. Graver, B. Servatius, H. Servatius, Combinatorial Rigidity, Graduate Studies in Mathematics, Vol. 2, American Mathematical Society, 1993.

[37] M.S. Gordon, Applications of Approximate Molecular Orbital Theory to Organic Molecules, Ph.D. Dissertation (directed by J.A. Pople), Department of Chemistry, Carnegie-Mellon University, 1968.

[38] A. Guichardet, On rotation and vibration motions of molecules, Ann. Inst. Henri PoincaréPhysique théorique, 40, 329-342, 1984. 
[39] J-C. Hausmann,A. Knutson, Polygon spaces and Grassmannians, L' Enseignement Mathèmatique, 43, 173-198, 1997.

[40] T.F. Havel, I. Najfeld, Applications of geometric algebra to the theory of molecular conformation 2. The local deformation problem, Theochem- J. Mol. Struc., 336, 175-189, 1995.

[41] S.Q. He, H.A. Scheraga, Macromolecular conformational dynamics in torsional angle space, J. Chem. Phys, 108, 271-286, 1998.

[42] W.J. Hehre, L. Radom, P.R. v. Schleyer, J.A. Pople, Ab initio Molecular Orbital Theory, Wiley, New York, 1986.

[43] IUPAC-IUB Commission on Biochemical Nomenclature, Abbreviations and Symbols for the Description of the Conformation of Polypeptide Chains, Biochemistry, 9, 3471-3479, 1970.

[44] IUPAC-IUB Joint Commission on Biochemical Nomenclature, Symbols for the Specifying the Conformation of Polysaccharide Chains, Eur. J. Biochem., 131, 5-7, 1983.

[45] IUPAC-IUB Joint Commission on Biochemical Nomenclature, Abbreviations and Symbols for the Description of Conformations of Polynucleotide Chains, Eur. J. Biochem., 131, 9-15, 1983.

[46] D.J. Jacobs, L.A. Kuhn, M.F. Thorpe, Flexible and Rigid Regions in Proteins, in Rigidity Theory and Applications, [85], 357-384, 1999.

[47] J.H. Jensen, K.K. Baldridge, M.S. Gordon, Uncatalyzed Peptide Bond Formation in the Gas Phase, J. Phys. Chem., 96, 8340-8351, 1992.

[48] M. Kapovich, J.J. Millson, The symplectic geometry of polygons in Euclidean space, Journal of Differential Geometry, 44, 479-513, 1996.

[49] H. Katayama, Building Geometric Models of Biological Molecules, Master's thesis in mathematics, University of South Carolina, 2003. c.f. http://www.math.sc.edu/ dix/katayama.

[50] K. Kawakubo, The Theory of Transformation Groups, Oxford University Press, Oxford, 1991.

[51] S. Kobayashi, K. Nomizu, Foundations of Differential Geometry, Volume I, John Wiley and Sons, New York, 1996.

[52] N.P. Landsman, Mathematical Topics Between Classical and Quantum Mechanics, Springer, New York, 1998.

[53] H.A. Lauwerier, A Note on Flexible Hexagons, Nederl. Akad. Wetensch. Proc. Ser. A, 69, 330-334, 1966.

[54] A. R. Leach,, Molecular Modelling: Principles and Applications, Second edition, Prentice Hall, Harlow, 2001.

[55] H.P.M. de Leeuw, C.A.G. Haasnoot, C. Altona, Empirical Correlations Between Conformational Parameters in $\beta$-D-Furanoside Fragments Derived from a Statistical Survey of Crystal Structures of Nucleic Acid Constituents, Israel J. Chem., 20, 108-126, 1980.

[56] S.H. Lee, K. Palmo, S. Krimm, A new formalism for molecular dynamics in internal coordinates, Chem. Phys., 265, 63-85, 2001.

[57] M. Levitt, A. Warshel, Extreme Conformational Flexibility of the Furanose Ring in DNA and RNA, J. Am. Chem. Soc., 100, no. 9, 2607-2613, 1978.

[58] R. Littlejohn, M. Reinsch, Gauge Fields in the Separation of Rotations and Internal Motions in the n-Body Problem, Reviews of Modern Physics, 69, no. 1, 213-275, 1997.

[59] H. Lodish, D. Baltimore, A. Berk, S.L. Zipursky, P. Matsudaira, J. Darnell, Molecular Cell Biology, Scientific American Books, Inc., New York, 1995.

[60] A.D. MacKerell Jr., N. Foloppe, All-Atom Empirical Force Field for Nucleic Acids, J. Computational Chem., 21, 86-120, 2000. See also the website: http://www.pharmacy.umaryland.edu/ alex/research.html

[61] D. Manocha, Y. Zhu, W. Wright, Conformational analysis of molecular chains using nanokinematics, Computer Applications in the Biosciences, 11, no. 1, 71-86, 1995.

[62] J.L. Markley, A. Bax, Y. Arata, C.W. Hilbers, R. Kaptein, B.D. Sykes, P.E. Wright, K. Wüthrich, Recommendations for the Presentation of NMR Structures of Proteins and Nucleic Acids, J. Mol. Biol., 280, (1998), 933-952.

[63] J.E. Marsden, Lectures on Mechanics, London Math. Soc. Lecture Note Series, 174, Cambridge University Press, Cambridge, 1992.

[64] C.K. Matthews, K.E. Van Holde, Biochemistry, second edition, Benjamin/Cummings, Menlo Park, 1996.

[65] C.J. Marzec, L.A. Day, An exact description of five-membered ring configurations. I Parameterization via an amplitude $S$, and angle $\Gamma$, the pseudorotation amplitude $q$ and phase angle 
$P$, and the bond lengths, Journal of Biomolecular Structure \& Dynamics, 10, no. 6, (1993), 1091-1123.

[66] A.K. Mazur, Symplectic Integration of Closed Chain Rigid Body Dynamics with Internal Coordinate Equations of Motion, J. Chem. Phys., 111, no. 4, 1407-1414, 1999.

[67] A.K. Mazur, R.A. Abagyan, New Methodology for Computer-aided Modelling of Biomolecular Structure and Dynamics 1. Non-cyclic Structures, 2. Local Deformations and Cycles, J. Biomolecular Struct. Dynamics, 6, no. 4, 815-845, 1989.

[68] J.M. McCarthy, Geometric Design of Linkages, Springer, New York, 2000.

[69] I. Motoc, R.A. Dammkoehler, Three-Dimensional Structure-Activity Relationships and Biological Receptor Mapping, in Mathematical and Computational Concepts in Chemistry, N. Trinajstic, ed., Ellis Horwood Limited, Chichester, 1986, 222-251.

[70] P.G. Mezey, Potential Energy Hypersurfaces, Studies in Physical and Theoretical Chemistry, Vol. 53, Elsevier, Amsterdam, 1987.

[71] J.R. Munkres, Elements of Algebraic Topology, Addison-Wesley, Menlo Park, California, 1984.

[72] A. Neumaier, Molecular Modeling of Proteins and Mathematical Prediction of Protein Structure, SIAM Rev., 39, no. 3, 407-460, 1997.

[73] See the website, http://ndb-mirror-2.rutgers.edu/NDB/archives/proj/valence/index.html

[74] See the website, http://www.rcsb.org/pdb

[75] D. Plavšić, M. Šoškić, Z. Daković, I. Gutman, A. Graovac, Extension of the Z Matrix to Cycle-Containing and Edge-Weighted Molecular Graphs, J. Chem. Inf. Comput. Sci., 37, 529-534, 1997.

[76] P. Pulay, G. Fogarasi, Geometry Optimization in Redundant Internal Coordinates, J. Chem. Phys., 96, no. 4, 2856-2860, 15 Feb 1992.

[77] C.C. Pye, R.A. Poirier, Graphical Approach for Defining Natural Internal Coordinates, J. Comp. Chem., 19, no. 5, 504-511, 1998.

[78] J.R. Quine, Helix Parameters and Protein Structure using Quaternions, Theochem- J. Mol. Struc., 460, 53-66, 1999. (See also the web site: http://www.math.fsu.edu/ ${ }^{\sim}$ quine for information on Discrete Frenet Frames).

[79] R. Randell, A Molecular Conformation Space, 125-140, and Conformation Spaces of Molecular Rings, 141-156, in MATH/CHEM/COMP 1987, R.C. Lacher (ed.), Studies in Physical and Theoretical Chemistry, Vol. 54, Elsevier, Amsterdam, 1988.

[80] W. Saenger, Principles of Nucleic Acid Structure, Springer-Verlag, New York, 1984.

[81] G. Schaftenaar, Molden, a package for displaying molecular density, http://www.cmbi.kun.nl/ schaft/molden/zmat/zmat.html, 1991.

[82] T. Schlick, Molecular Modeling and Simulation, Springer, New York, 2002.

[83] A.R. Srinivasan, W.K. Olson, Nucleic Acid Model Building: The Multiple Backbone Solutions Associated with a Given Base Morphology, J. Biomolecular Struct. Dynamics, 4, no. 6, 895938, 1987.

[84] R. Stanley, Enumerative Combinatorics, Vol. I, Cambridge Univ. Press, Cambridge, 1997.

[85] M. Thorpe, P. Duxbury, eds., Rigidity Theory and Applications, Vol. I and II, Kluwer Academic/Plenum Publishers, New York, 1999.

[86] N. Trinajstić, Chemical Graph Theory, Vol. I and II, CRC Press, Boca Raton, 1983.

[87] D.B. West, Introduction to Graph Theory, Prentice Hall, Upper Saddle River, NJ, 1996.

[88] W. Whiteley, Representing Geometric Configurations, in Learning and Geometry: Computational Approaches, Progress in Computer Science and Applied Logic, Vol. 14, Birkhäuser, Boston, 1996, 143-178.

[89] W. Whiteley, Rigidity of Molecular Structures: Generic and Geometric Analysis, in Rigidity Theory and Applications, [85], 21-46, 1999.

[90] E.B. Wilson, J.C. Decius, P.C. Cross Molecular Vibrations, McGraw-Hill, New York, 1955.

[91] K. Wirth, Coding of Relational Descriptions of Molecular Structures, J. Chem. Inf. Comput. Sci., Vol. 26, 242-249, 1986.

[92] T. Zaslavski, Perpendicular dissections of space, Discrete Comput. Geom.,27, 303-351, 2002.

Department of Mathematics, University of South Carolina

E-mail address: dix@math.sc.edu 